CLNS 91/1121

May 1992

\title{
Low-Lying States of the Six-Dimensional Fractional Superstring
}

\author{
Philip C. Argyres', Edwin Lyman' and S.-H. Henry Tye \\ Newman Laboratory of Nuclear Studies \\ Cornell University \\ Ithaca, N.Y. 14853-5001
}

\begin{abstract}
The $K=4$ fractional superstring Fock space is constructed in terms of $\mathbf{Z}_{4}$ parafermions and free bosons. The bosonization of the $\mathbf{Z}_{4}$ parafermion theory and the generalized commutation relations satisfied by the modes of various parafermion fields are reviewed. In this preliminary analysis, we describe a Fock space which is simply a tensor product of $\mathbf{Z}_{4}$ parafermion and free boson Fock spaces. It is larger than the Lorentz-covariant Fock space indicated by the fractional superstring partition function. We derive the form of the fractional superconformal algebra that may be used as the constraint algebra for the physical states of the FSS. Issues concerning the associativity, modings and braiding properties of the fractional superconformal algebra are also discussed. The use of the constraint algebra to obtain physical state conditions on the spectrum is illustrated by an application to the massless fermions and bosons of the $K=4$ fractional superstring. However, we fail to generalize these considerations to the massive states. This means that the appropriate constraint algebra on the fractional superstring Fock space remains to be found. Some possible ways of doing this are discussed.
\end{abstract}

\footnotetext{
*E-mail address: pca@strange.tn.cornell.edu; pca@crnlnuc.bitnet

${ }^{\dagger}$ E-mail address: lyman@beauty.tn.cornell.edu
} 


\section{Introduction}

String theory [1] is the only known theory with the potential for describing all matter and forces in nature in a unified way. In particular, the superstring and the closely related heterotic string entail many structures, including gravity, Yang-Mills fields and chiral fermions, that are central to our present understanding of the world. However, their critical space-time dimension is ten, and though there are numerous proposed mechanisms to reduce the number of observable dimensions, there is no known compelling reason why the superstring theory should have only four large space-time dimensions. While it is important to search for dynamical and/or symmetry reasons explaining how our world could be realized in the heterotic/superstring framework, we would like to ask instead if other string theories with lower critical dimensions exist, for they could provide more natural descriptions of the world.

Recently strong evidence has been presented for the existence of such string theories [2]. Since string theories are characterized by the local symmetries of a twodimensional conformal field theory on the string world-sheet, it is natural to try to construct string theories with smaller critical space-time dimensions by changing the world-sheet symmetry. It is well-known that fractional-spin fields exist in two-dimensional theories. One can imagine new local symmetries on the world-sheet which involve fractional-spin currents (replacing the spin-3/2 supercurrent of the superstring) and which lead to string propagation in space-times with dimensions less than 10. In ref. [2], string theories, called fractional superstrings (FSS), with spin 4/3 and $6 / 5$ currents on the world-sheet were found to have potentially interesting phenomenologies in 6 and 4 critical space-time dimensions, respectively. In this paper, we discuss in detail the spectrum and physical state conditions of the 6-dimensional FSS. Although the results obtained are unsatisfactory (or, at best, incomplete), we believe the analysis presented below illuminates some of the main issues that are involved in understanding the FSS.

The basic idea behind the FSS is to replace the world-sheet supersymmetry of the superstring theory with a world-sheet fractional supersymmetry parametrized by an integer $K \geq 2$. Such a fractional supersymmetry relates world-sheet bosons not to fermions but rather to world-sheet parafermions. The world-sheet fractional superpartner of the space-time coordinate $X^{\mu}$ is a field $\epsilon^{\mu}$ of spin $2 /(K+2)$. This field is the so-called "energy operator" of the $\mathbf{Z}_{K}$ parafermion theory [3]. The fractional supersymmetry is generated by a generalization of the supercurrent, a new chiral current $G$ [4, 5, 6, 7, 8] whose conformal dimension is $(K+4) /(K+2)$. This new current transforms $X^{\mu}$ to the fractional-spin field $\epsilon^{\mu}$.

By demanding that the FSS have only transverse propagating modes, a generalization of an argument of Brink and Nielson [9] implies that the critical dimensions of such string theories should be [2]

$$
D=2+16 / K \text {. }
$$


The case $K=2(D=10)$ corresponds to the superstring. The new theories are those with $K>2$; for $K=4,8$ and 16 we have the integer critical dimensions $D=6,4$ and 3 , respectively.

In this paper we will concentrate exclusively on the simplest case after the $(K=2)$ superstring, the $K=4 \mathrm{FSS}$. The reasons for this are twofold. The first is that the complexity of these theories increases considerably with increasing $K$. Although the world-sheet fractional supersymmetry algebra is non-local, the $\mathbf{Z}_{4}$ parafermion fields that appear in the $K=4$ FSS can be simply represented by free bosons, which enables the calculations to be simplified tremendously. This is not the case in the $K=8$ and $K=16$ theories [10, 11]. Furthermore, a close examination shows that the appropriate world-sheet fractional supersymmetry algebra for the $K=8$ theory contains two spin- $13 / 5$ currents in addition to the spin-6/5 current [1], which further complicate the analysis. The second reason for our emphasis on the $K=4$ FSS is because it is potentially the most interesting one from the phenomenological point of view. As argued in [12, 13], the requirements of quantum mechanics, Lorentz invariance and locality suggest that the $K=4$ FSS may be automatically compactified from six to four space-time dimensions. Furthermore, as argued in [12], the compactification from the critical dimension 6 to the natural dimension 4 offers the possibility of the construction of heterotic type $K=4$ FSS models that have chiral space-time fermions. This is encouraging, since the $K=4$ FSS, because of its relative simplicity, affords the best prospect for detailed examination in the near future.

Let us highlight some of the similarities and differences between the $K=4$ FSS and the superstring. In the superstring, the world-sheet superpartner of the space-time coordinate boson $X^{\mu}$ is a Majorana fermion $\psi^{\mu}$. $\psi$ is the primary field of dimension $1 / 2$ in the Ising model, the $c=1 / 2$ minimal unitary conformal field theory (CFT) [14]. This theory has two other primary fields, the identity $\mathbb{1}$ and the spin field $\sigma$, which play specific roles in the construction of the superstring Fock space. All Neveu-Schwarz (space-time bosonic) states can be generated by the action of the modes of the Majorana fermion field on the identity, and all states in the Ramond sector (space-time fermions) can be generated by the action of the modes of the Majorana fermion on the spin field.

In the $K=4$ string, on the other hand, the world-sheet fractional superpartner of the space-time coordinate boson is the dimension-1/3 energy operator $\epsilon^{\mu}$, a primary field in the $\mathbf{Z}_{4}$ parafermion theory. This CFT has central charge $c=1$ and an infinite number of primary fields. In Sect. 2 we develop a Fock space description of the $\mathbf{Z}_{4}$ parafermion theory, and show how the states are divided into sectors that close under the action of the energy operator modes. Different sectors will be seen to correspond to space-time bosons and fermions, in analogy to the Neveu-Schwarz and Ramond sectors of the superstring. The analysis of the $\mathbf{Z}_{4}$ parafermion theory is made easier by the fact that the $\mathbf{Z}_{4}$ parafermion theory can be realized as a free boson $\phi$ compactified on a circle of radius 2 (where the boson is normalized by $\langle\rho(z) \rho(0)\rangle=-\frac{2}{3} \ln z$ ) [15]. 
Nevertheless a number of technical issues having to do with the modings of fractionalspin operators on the world-sheet will have to be unraveled. In this paper we consider the simplest case, where the Fock space of the open $K=4$ FSS is described as the $D$-fold tensor product of the $\mathbf{Z}_{4}$ parafermion Fock spaces and free coordinate boson Fock spaces, with $D$ the dimension of space-time.

At a fundamental level, the superstring is described by its world-sheet gauge invariance - two-dimensional superconformal supergravity. Classically, the gauge fixing constraints in the superconformal gauge consist of the vanishing of the energymomentum tensor $T(z)$ and the supercurrent $G_{s}(z)=\psi^{\mu} \partial X_{\mu}$. Upon quantization, these constraints are realized weakly on the space of physical states of the superstring:

$$
\langle\phi|T(z)| \chi\rangle=\left\langle\phi\left|G_{s}(z)\right| \chi\right\rangle=0
$$

for all physical states $\phi$ and $\chi$. By writing $T$ and $G_{s}$ in modes, these conditions can be factorized into the familiar physical state conditions, in which the positive modes of $T$ and $G_{s}$ annihilate physical states. We have no understanding at present of the classical world-sheet gauge invariance of the $K=4 \mathrm{FSS}$. However, there does exist a natural analog of the supercurrent $G_{s}$, the dimension $4 / 3$ fractional supercurrent $G$ of the form

$$
G(z) \sim \epsilon^{\mu} \partial X_{\mu}+\ldots .
$$

The precise expression for $G(z)$ will be explored in Sect. 3, where we will also discuss some aspects of the algebra satisfied by $G$. A basic assumption that will be made in this paper is that there exists some sort of "fractional superconformal gauge" whose classical constraint equation is the vanishing of $G$ (and $T$, of course - we still require two-dimensional reparametrization and Weyl invariance). One goal in this paper is to formulate and solve, at the massless levels, the resulting FSS physical state conditions.

In Sect. 4 , we begin our exploration of the FSS constraint algebra by solving the physical state conditions for the massless propagating modes of the open $K=4$ FSS in the space-time fermion sector. We show how space-time spinors are naturally described in the FSS Fock space, and that the massless state is a Weyl fermion. We find that there exists a natural left-right pairing of massive states in the fermionic sector necessary for Lorentz invariance. The existence of this pairing for all mass levels follows from a counting argument relying on the Euler pentagonal number theorem.

We turn to an examination of the physical states in the bosonic sector in Sect. 5 . The open FSS massless propagating modes turn out to describe a massless vector particle. A novel feature compared to the superstring is the existence of normal-ordering constant ("intercept") for the fractional supercurrent physical state condition in the bosonic sector.

Sect. 6 describes two problems that appear upon solving the physical state conditions at higher mass levels of the open FSS Fock space. These are, firstly, that the 
tensor product Fock space description of states is Lorentz non-covariant above the massless level, and, secondly, that the physical state consitions do not remove nearly enough states to agree with the counting of states in the FSS partition function. We do not have a clear resolution of these problems; however, they suggest that the tensor product Fock space described in this paper is larger than the Lorentz-covariant space of states implied by the FSS partition function. One can either search for a direct way of reducing the tensor-product Fock space to one in which the physical state conditions have a Lorentz-covariant action, or modify/supplement the physical state conditions to reduce the number of propagating degrees of freedom and cancel the Lorentz non-covariant terms in the equations of motion.

In Sect. 7 we remark on two features of the $K=4$ FSS which may provide useful hints for finding a complete and correct description of the FSS space of states. First, we discuss the possible equivalence of the $K=4$ FSS with the spin- $4 / 3$ string introduced in ref. [8]. The critical central charge of the spin- $4 / 3$ string, unlike the $K=4$ string, can be calculated by constructing extra towers of null states using the algebra of physical state conditions. A representation of the spin- $4 / 3$ algebra would then give a non-tensor-product realization of the FSS space of states. However, the representation theory of this algebra is not well understood, and thus a direct comparison of its spectrum of physical states with the $K=4$ FSS partition function cannot be made yet. Second, we show that a chiral closed $K=4$ FSS must have extra massless scalars in its spectrum if gravitational anomalies are to cancel.

We have collected some of the more technical or tangential discussions in a series of appendices. Various chiral associative solutions to the $\mathbf{Z}_{4}$ parafermion theory are constructed using cocycles in Appendix A. A review of the derivation of the generalized commutation relations satisfied by fractional-spin fields, based on examples taken from the $\mathbf{Z}_{4}$ parafermion theory is presented in Appendix B. Appendix O discusses the representation theory and associativity constraints of the fractional superconformal algebra. In Appendix D we review the construction of the modular invariant partition function for the closed $K=4 \mathrm{FSS}$. This makes the analog of the GSO projection [16] in the FSS apparent, and aids in the identification of bosonic and fermionic states in the FSS Fock space. Finally, in Appendix E we discuss the physical state conditions and null states of the spin- $4 / 3$ string.

\section{The $K=4$ FSS Fock Space}

Each space-time dimension of the $K=4$ FSS corresponds to a free coordinate boson $X^{\mu}$ and a $\mathbf{Z}_{4}$ parafermion theory on the string world-sheet. In this section we will construct a free-field representation of the $\mathbf{Z}_{4}$ parafermion theory, first pointed out by Yang 15. We use this representation to compute the operator product expansions (OPEs), mode expansions, generalized commutation relations, and characters of primary fields in the parafermion theory. In this way we will be able to build up a Fock space realization of the space of states for each dimension of the FSS. We 
assume that the total Fock space is a tensor product of $D$ of these individual Fock spaces. This tensor product structure will be the subject matter of later sections.

\section{$2.1 \quad$ Review of $\mathrm{Z}_{4}$ parafermions}

The operator content of the chiral $\mathbf{Z}_{4}$ parafermion theory can be realized by the $S U(2)_{4} / U(1)$ coset model [3]. The chiral $S U(2)_{4}$ WZW theory [4] has central charge $c_{\mathrm{WZW}}=2$ and consists of holomorphic primary fields $\Phi_{m}^{j}(z)$ of conformal dimension $j(j+1) / 6$. The indices $j, m \in \mathbf{Z} / 2$ label $S U(2)$ representations where $0 \leq j \leq 2$ and $|m| \leq j$ with $j-m \in \mathbf{Z}$. When we factor a $U(1)$ subgroup out of $S U(2)_{4}$, we correspondingly factor the primary fields as

$$
\Phi_{m}^{j}(z)=\phi_{m}^{j}(z) \exp \left\{i \frac{m}{2} \varphi(z)\right\}
$$

Here $\varphi$ is the free $U(1)$ boson normalized so that $\langle\varphi(z) \varphi(w)\rangle=-2 \ln (z-w)$. The $\phi_{m}^{j}(z)$ are Virasoro primary fields in the $\mathbf{Z}_{4}$ parafermion theory with conformal dimensions:

$$
\Delta\left(\phi_{m}^{j}\right)=\frac{j(j+1)}{6}-\frac{m^{2}}{4} \quad \text { for }|m| \leq j .
$$

The central charge of the $\mathbf{Z}_{4}$ parafermion theory is then $c=c_{\mathrm{WZW}}-c_{\varphi}=1$. This is an indication that the $\mathbf{Z}_{4}$ parafermion can be realized by a free boson. The definition of the $\phi_{m}^{j}$ fields can be consistently extended to the case where $|m|>j$ by the rules

$$
\phi_{m}^{j}=\phi_{m+4}^{j}=\phi_{m-2}^{2-j} .
$$

With these identifications, an independent set of fields can be taken to be $\phi_{0}^{0}, \phi_{ \pm 1}^{0}$, $\phi_{ \pm 1 / 2}^{1 / 2}, \phi_{0}^{1}, \phi_{1}^{1}, \phi_{ \pm 1 / 2}^{3 / 2}$ and $\phi_{0}^{2}$.

The fusion rules of the parafermion fields follow from those of the $S U(2)_{4}$ theory:

$$
\left[\phi_{m_{1}}^{j_{1}}\right] \otimes\left[\phi_{m_{2}}^{j_{2}}\right] \sim \sum_{j=\left|j_{1}-j_{2}\right|}^{r}\left[\phi_{m_{1}+m_{2}}^{j}\right]
$$

where $r=\min \left\{j_{1}+j_{2}, 4-j_{1}-j_{2}\right\}$. The sectors $\left[\phi_{m}^{j}\right]$ include the primary fields $\phi_{m}^{j}$ and a tower of higher-dimension fields (with dimensions differing by integers) defined as in (2.1) from current algebra descendants of the $\Phi_{m}^{j}$. Indeed, the $S U(2)_{4}$ currents factorize as well:

$$
\begin{aligned}
J^{+} & =2 \psi_{1} e^{i \varphi / 2} \\
J^{0} & =i \partial \varphi \\
J^{-} & =2 \psi_{-1} e^{-i \varphi / 2}
\end{aligned}
$$

where the parafermion currents $\psi_{\ell} \equiv \phi_{\ell}^{0}=\phi_{\ell-2}^{2}$ and $\psi_{-\ell} \equiv \psi_{4-\ell}$ have conformal dimensions $\ell(4-\ell) / 4$ in accordance with (2.2). It follows from the fusion rules 
(2.4) that the current blocks $\left[\psi_{\ell}\right], \ell=0,1,2,3$ form a closed algebra, namely, the $\mathbf{Z}_{4}$ parafermion current algebra. Note that $\psi_{1}$ acting on a field $\phi_{m}^{j}$ increases the $m$ quantum number by one but does not change the $S U(2)$ spin $j$.

Another special field - the one that will play the central role in the construction of the FSS Fock space to follow - is the energy operator $\epsilon \equiv \phi_{0}^{1}$ of dimension 1/3. Operating on a field $\phi_{m}^{j}$ with $\epsilon$ preserves the $m$ quantum number but yields sectors with $j$ quantum numbers $j-1, j$, and $j+1$, when permitted by the fusion rule (2.4).

Before plunging into the detailed construction of the parafermion Fock space, we can already make some important remarks concerning the roles the various parafermion fields can be expected to play in the FSS. The energy operator $\epsilon$, as the fractional superpartner of the coordinate boson field $X$, will play a role analogous to the one the Majorana fermion field $\psi$ plays in the superstring. There, the Neveu-Schwarz sector is built from the action of $\psi$ on the identity. From the fusion rules (2.4) we see that $\epsilon$ acting on the identity can create all parafermions with $m$ quantum number zero. Thus, we expect that the set of fields $\left\{\left[\phi_{0}^{j}\right]\right\}$ will be the analog of the Neveu-Schwarz sector in the FSS. Just as the Ramond sector is generated by the action of $\psi$ on another field, we should look for other sectors in the parafermion theory that close under the action of $\epsilon$. Because of the identifications in (2.3), there are only three such other sectors: $\left\{\left[\phi_{ \pm 1 / 2}^{j}\right]\right\}$ and $\left\{\left[\phi_{1}^{j}\right]\right\}$. These are formed by the action of $\epsilon$ on the so-called "spin" fields of the parafermion theory, $\sigma_{ \pm 1} \equiv \phi_{ \pm 1 / 2}^{1 / 2}$ and $\sigma_{2} \equiv \phi_{1}^{1}$, respectively. The arguments of Appendix D indicate that the $m=1$ sector plays the role of the Ramond sector in the FSS, while the $m= \pm 1 / 2$ sectors do not enter into the FSS Fock space at all. The fusion rules (2.4) show that it is consistent to project out the half-odd integral spin states since the integral spin states do not close on them; Appendix D shows that this projection is required by modular invariance.

\subsection{Bosonization of $\mathrm{Z}_{4}$ parafermions}

The spectrum of the $c=1 \mathbf{Z}_{4}$ parafermion theory is the same as that of the $\mathbf{Z}_{2}$ orbifold of a boson on a circle of a certain radius 15. In line with the above discussion, we will ignore the parafermion fields with spin $j \in \mathbf{Z}+\frac{1}{2}$ which correspond to the twist fields in the orbifold theory. Therefore we will just be interested in a free boson taking values on a circle. This bosonization will allow us to easily construct the Fock space of the parafermion theory and to derive a simple form for the parafermion characters as sums over the winding modes of the compactification.

Consider a chiral boson $\rho(z)$ satisfying

$$
\langle\rho(z) \rho(w)\rangle=-\frac{2}{3} \ln (z-w) .
$$

With this choice of normalization, the energy-momentum tensor is given by

$$
T_{\rho}(z)=-\frac{3}{4}:(\partial \rho(z))^{2}:
$$


If we take this boson to be compactified on a circle of radius 2 [in the units implied by (2.6)], so that $\rho=\rho+4 \pi n, n \in \mathbf{Z}$, the Virasoro primary fields include the dimension 1 field $i \partial \rho(z)$, higher dimension fields built from it, and the infinite set of fields

$$
\epsilon^{(a)}(z)=: \mathrm{e}^{i a \rho(z)}:, \quad a \in \mathbf{Z} / 2,
$$

with conformal dimensions

$$
\Delta\left(\epsilon^{(a)}\right)=\frac{a^{2}}{3} .
$$

Comparing to the conformal dimensions of the $\phi_{m}^{j}$ parafermion fields (2.2), we make the identifications

$$
\begin{aligned}
& \epsilon^{(0)}(z)=\phi_{0}^{0}(z) \quad(=\mathbb{1}), \quad \Delta=0, \\
& \epsilon^{( \pm 1 / 2)}(z)=\phi_{1}^{1}(z) \quad\left(=\sigma_{2}\right), \quad \Delta=1 / 12, \\
& \epsilon^{( \pm 1)}(z)=\phi_{0}^{1}(z) \quad(=\epsilon), \quad \Delta=1 / 3, \\
& \epsilon^{( \pm 3 / 2)}(z)=\phi_{ \pm 1}^{0}(z)\left(=\psi_{ \pm 1}\right), \quad \Delta=3 / 4, \\
& i \partial \rho(z)=\phi_{0}^{2}(z) \quad\left(=\psi_{2}\right), \quad \Delta=1,
\end{aligned}
$$

where we have also given in parentheses the corresponding spin-field, energy operator, or parafermion current symbol. The identification of fields in the free boson theory with $\mathbf{Z}_{4}$ parafermion fields is discussed in more detail in Appendix A.

The OPEs of these fields are easily computed to be, for $a+b \neq 0$,

$$
\epsilon^{(a)}(z) \epsilon^{(b)}(w)=(z-w)^{2 a b / 3}\left[\epsilon^{(a+b)}(w)+\frac{a}{a+b}(z-w) \partial \epsilon^{(a+b)}+\ldots\right],
$$

or, if $a+b=0$,

$$
\epsilon^{(a)}(z) \epsilon^{(-a)}(w)=(z-w)^{-2 a^{2} / 3}[\mathbb{1}+a(z-w) i \partial \rho(w)+\ldots] ;
$$

and

$$
i \partial \rho(z) \epsilon^{(a)}(w)=\frac{2 a}{3} \frac{1}{(z-w)} \epsilon^{(a)}(w)+\frac{1}{a} \partial \epsilon^{(a)}(w)+\ldots
$$

A special feature of the free boson representation is its $\mathbf{Z}_{3}$ symmetry. We can associate a $\mathbf{Z}_{3}$ charge $q$ with the $\epsilon^{(a)}$ fields by the rule

$$
q \equiv-2 a(\bmod 3), a \in \mathbf{Z} / 2 .
$$

Thus the parafermion sectors $\left[\phi_{m}^{j}\right]$ with $j=0$ or 2 have $\mathbf{Z}_{3}$ charge $q=0$, while the $j=1$ sectors have $q= \pm 1$. From the OPEs it follows that this charge is additive under fusion. We will see below that its occurrence makes for important technical simplifications. Note that the $\mathbf{Z}_{3}$ symmetry of this free boson representation has nothing to do with the $\mathbf{Z}_{4}$ symmetry of the parafermion current algebra - its existence 
is "accidental" in the sense that there do not in general exist $\mathbf{Z}_{N}$ symmetries of the whole spectrum of other $\mathbf{Z}_{K}$ parafermion theories.

The Fock space of a free boson compactified on a circle is usually built up by the action of the modes of the boson field. The (chiral) mode expansion of $\rho(z)$ is

$$
\rho(z)=r_{0}-i s_{0} \ln z+i \sum_{n \neq 0} \frac{1}{n} s_{n} z^{-n},
$$

where the modes satisfy the commutation relations following from (2.6)

$$
\left[r_{0}, s_{0}\right]=\frac{2 i}{3}, \quad\left[s_{n}, s_{m}\right]=\frac{2 n}{3} \delta_{n+m} .
$$

The Fock space is built with the $\rho(z)$ creation operators from the vacuum $|0\rangle$ satisfying $s_{m}|0\rangle=0$ for $m \geq 0$. Because $\rho(z)$ is compactified on a circle of radius 2 ,

$$
r_{0}=r_{0}+4 \pi n, \quad n \in \mathbf{Z} .
$$

In the zero-mode sector the allowed $s_{0}$ eigenstates are therefore

$$
|a\rangle \equiv \mathrm{e}^{i a r_{0}}|0\rangle, \quad a \in \mathbf{Z} / 2,
$$

satisfying

$$
s_{0}|a\rangle=\frac{2 a}{3}|a\rangle
$$

Clearly, these states $|a\rangle$ are created from the vacuum by the vertex operators $\epsilon^{(a)}(z)$ :

$$
|a\rangle=\epsilon^{(a)}(0)|0\rangle .
$$

The rest of the Fock space is built up by acting on the $|a\rangle$ states with the $s_{m}$ modes with $m<0$.

From this description of the Fock space, and the identifications (2.10) of the parafermion operators in each sector, we can easily derive an expression for the character $\mathcal{Z}_{m}^{j}$ of the parafermion sector $\left[\phi_{m}^{j}\right]$. Indeed, each parafermion sector $\left[\phi_{m}^{j}\right]$ consists of the primary field $\phi_{m}^{j}$ and its parafermion and Virasoro descendants whose dimensions differ by integers. In the bosonic Fock space, these sectors correspond to the "momentum" state $|a\rangle$ identified in (2.10), its associated winding states $|a+3 n\rangle$, and all their descendants created by the action of the $s_{m}$ modes. The counting of the $s_{m}$ descendants simply contributes a factor of the free boson partition function $\eta(q)^{-1}$ to the parafermion character, where

$$
\eta(q)=q^{1 / 24} \prod_{n=1}^{\infty}\left(1-q^{n}\right)
$$

is the Dedekind $\eta$-function. Here $q=e^{2 \pi i \tau}$, where $\tau$ is the complex modulus on the torus. Since the character is defined to be $\operatorname{Tr} q^{L_{0}}$ over each sector, the factor of $\eta(q)^{-1}$ 
must be multiplied by $q^{\Delta}$ where $\Delta$ is the dimension of each winding state $|a+3 n\rangle$ appearing in $\left[\phi_{m}^{j}\right]$. Thus, we find the characters

$$
\begin{aligned}
\mathcal{Z}_{0}^{0}+\mathcal{Z}_{0}^{2} & =\frac{1}{\eta}\left(\sum_{n=-\infty}^{\infty} q^{3 n^{2}}\right) \\
\mathcal{Z}_{1}^{1} & =\frac{q^{1 / 12}}{\eta}\left(\sum_{n=-\infty}^{\infty} q^{3 n^{2}+n}\right), \\
\mathcal{Z}_{0}^{1} & =\frac{q^{1 / 3}}{\eta}\left(\sum_{n=-\infty}^{\infty} q^{3 n^{2}+2 n}\right), \\
\mathcal{Z}_{1}^{0}=\mathcal{Z}_{-1}^{0} & =\frac{q^{3 / 4}}{\eta}\left(\sum_{n=0}^{\infty} q^{3 n^{2}+3 n}\right) .
\end{aligned}
$$

These characters will form the basis for our discussion of modular invariance of the closed $K=4$ FSS in Appendix D.

\subsection{Mode expansions and commutation relations}

Though the description of the $\mathbf{Z}_{4}$ parafermion Fock space given above is complete, it is not expressed in the right language for our purposes. We will need to define the mode expansions of the $\epsilon^{(a)}$ fields and derive the commutation relations these modes satisfy in order to state and solve the FSS physical state conditions. Because the parafermion fields have fractional spin, the usual contour deformation argument [14] for deriving commutation relations of modes from the OPE of the fields must be modified. For fields that satisfy abelian braid relations, as the $\epsilon^{(a)}$ do, Zamolodchikov and Fateev [3] have invented the necessary modifications. Their construction is reviewed in Appendix B. The result is that abelian braided fields satisfy generalized commutation relations (GCRs) which involve infinite sums of terms in place of the usual commutator or anticommutator.

First, though, we must introduce the mode expansions of the $\epsilon^{(a)}$. From the OPEs (2.11) $-(2.13)$ we see that in general

$$
\epsilon^{(a)}(z) \chi_{q}(0)=\sum_{n \in \mathbf{Z}} z^{n-a q / 3} \chi_{q+q_{a}}^{(n)}(0),
$$

where $\chi_{q}$ represents any field with $\mathbf{Z}_{3}$ charge $q$, and $q_{a}$ is the $\mathbf{Z}_{3}$ charge of $\epsilon^{(a)}$. The $\chi_{q+q_{a}}^{(n)}$ fields are all the primary and descendant fields that appear on the right-hand side of the OPE. From 2.23, the dimension of $\chi_{q+q_{a}}^{(n)}$ is $\Delta\left(\chi_{q+q_{a}}^{(n)}\right)=\Delta\left(\chi_{q}\right)+n+a(a-$ $q) / 3$, where we have used $\Delta\left(\epsilon^{(a)}\right)=a^{2} / 3$. Following the usual convention that the subscript on a mode operator is the negative of its dimension, we define the $\epsilon^{(a)}$ modes by

$$
\chi_{q+q_{a}}^{(n)}(0) \equiv \epsilon_{-n+a(q-a) / 3}^{(a)} \chi_{q}(0) .
$$


Thus the general OPE (2.23) can be written

$$
\epsilon^{(a)}(z) \chi_{q}(0)=\sum_{n} z^{n-a q / 3} \epsilon_{-n+a(q-a) / 3}^{(a)} \chi_{q}(0)
$$

or

$$
\epsilon_{n+a(q-a) / 3}^{(a)} \chi_{q}(0)=\oint_{\gamma} \frac{\mathrm{dz}}{2 \pi i} z^{n-1+a q / 3} \epsilon^{(a)}(z) \chi_{q}(0)
$$

where $\gamma$ is a contour encircling the $\chi_{q}(0)$ insertion once. Because the OPE (2.23) describes abelian braiding (there is only one cut on the right hand side) it was possible to choose the integrand in (2.26) so that the contour of integration closes. In the case of non-abelian braiding this is not in general possible.

From the OPEs of the fields we can now derive their GCRs following the discussion in Appendix B. However, as explained there, there are actually an infinite number of these relations which can be obtained from a single OPE depending on how many terms on the right hand side of the OPE are included. Of course, these GCRs are all consistent, but those encoding more terms of an OPE contain more information. We will find in the following analysis that only the first few terms of the $\mathbf{Z}_{4}$ parafermion OPEs are sufficient to determine all the mode relations in the parafermion Fock space.

We can choose in deriving these relations to select only the first term on the righthand side of the $\epsilon^{(a)} \epsilon^{(b)}$ OPEs (2.11) and (2.12) by multiplying them by the factor $(z-w)^{\alpha}$ with $\alpha=-1-2 a b / 3$. Then, following the procedure outlined in Appendix B, we obtain the GCR

$$
\begin{gathered}
\sum_{\ell=0}^{\infty} c_{\ell}^{(\alpha)}\left[\epsilon_{n-\ell-1+\frac{a(q-2 b-a)}{3}}^{(a)} \epsilon_{m+\ell+1+\frac{b(q-b)}{3}}^{(b)}+\epsilon_{m-\ell+\frac{b(q-2 a-b)}{3}}^{(b)} \epsilon_{n+\ell+\frac{a(q-a)}{3}}^{(a)}\right] \\
=\epsilon_{n+m+\frac{(a+b)(q-a-b)}{3}}^{(a+b)}
\end{gathered}
$$

The $c_{\ell}^{(\alpha)}$ are the binomial coefficients defined by $(1-x)^{\alpha}=\sum_{\ell=0}^{\infty} c_{\ell}^{(\alpha)} x^{\ell}$. The above expressions are understood to be valid only when acting on a state with $\mathbf{Z}_{3}$ charge $q$. Note that for $a+b=0$, the right hand side is $\epsilon_{n+m}^{(0)}=\delta_{n+m}$. From the $\rho \epsilon$ OPE (2.11), a standard commutator is obtained:

$$
\left[s_{n}, \epsilon_{m+a(q-a) / 3}^{(a)}\right]=\frac{2 a}{3} \epsilon_{n+m+a(q-a) / 3}^{(a)} .
$$

As we will see, it turns out that this relation is insufficient for our analysis. We will also need the GCR corresponding to keeping one more term in the $\rho \epsilon \mathrm{OPE}$,

$$
\begin{array}{r}
\sum_{\ell=0}^{\infty}\left[s_{n-\ell-1} \epsilon_{m+\ell+1+a(q-a) / 3}^{(a)}+\epsilon_{m-\ell+a(q-a) / 3}^{(a)} s_{n+\ell}\right] \\
=\left\{\frac{2 a n}{3}-\frac{1}{a}\left(n+m+\frac{a q}{3}\right)\right\} \epsilon_{n+m+a(q-a) / 3}^{(a)} .
\end{array}
$$


Note that (2.28) can be derived from (2.29).

These generalized commutators may seem complicated and difficult to use due to the infinite summation. However, when acting on any given state only a finite number of terms from the infinite sum contributes. This occurs because, as the summation index $\ell$ gets larger, the dimension of the right-most operators in the GCRs become more and more negative, so that eventually they annihilate any given state. For example, the vacuum satisfies the properties

$$
\begin{aligned}
\epsilon_{n-a^{2} / 3}^{(a)}|0\rangle & =0, \quad n>0, \\
s_{n}|0\rangle & =0, \quad n \geq 0,
\end{aligned}
$$

following from the definition of the modes. Note that the definition of the "momentum" states (2.20) implies

$$
\epsilon_{-a^{2} / 3}^{(a)}|0\rangle=|a\rangle \text {. }
$$

Starting from (2.30) and (2.31) the GCRs (2.27)-(2.29) are sufficient to build up the whole Fock space of the $\mathbf{Z}_{4}$ parafermion theory using $\epsilon^{(a)}$ modes.

To translate between the description of the Fock space in terms of the $s_{m}$ modes presented in the last subsection, and the $\epsilon^{(a)}$ modes being discussed here, we need to derive a relation of the form

$$
\epsilon_{r}^{(a)}|b\rangle=P_{r}^{(a)}(s)|a+b\rangle,
$$

where $P_{r}^{(a)}(s)$ is a polynomial in the boson creation modes $s_{m}$ with $m<0$. Then, using the $s_{m}-\epsilon_{r}^{(a)}$ commutation relation (2.28), an arbitrary $\epsilon$-state can be translated to the $s_{m}$ Fock space description:

$$
\prod_{j=1}^{N} \epsilon_{r_{j}}^{\left(a_{j}\right)}\left|a_{0}\right\rangle=P(s)\left|\sum_{j=0}^{N} a_{j}\right\rangle,
$$

for some definite polynomial $P$.

We will now use the GCRs to derive a recursive formula for the polynomial $P_{r}^{(a)}(s)$ in (2.32). This will also serve as an example of the use of the GCRs. Consider the GCR (2.27) with $m=-1$ acting on the vacuum $|0\rangle$, so that $q=0$. Using the vacuum properties (2.30) and (2.31), we obtain

$$
\begin{aligned}
\epsilon_{n-a(a+2 b) / 3}^{(a)}|b\rangle & =0, \quad n>0, \\
\epsilon_{-a(a+2 b) / 3}^{(a)}|b\rangle & =|a+b\rangle .
\end{aligned}
$$

Now consider the $s_{m}-\epsilon_{r}^{(a)}$ GCR (2.29) acting on the state $|b\rangle$. By the $\mathbf{Z}_{3}$ charge assignments (2.14) $q=-2 b$, and if we choose $n=0$ and $m=-k<0$, we obtain

$$
\begin{gathered}
\left(\sum_{\ell=0}^{\infty} s_{-\ell-1} \epsilon_{\ell+1-k-a(a+2 b) / 3}^{(a)}\right)|b\rangle+\epsilon_{-k-a(a+2 b) / 3}^{(a)} s_{0}|b\rangle= \\
\left(\frac{k}{a}+\frac{2 b}{3}\right) \epsilon_{-k-a(a+2 b) / 3}^{(a)}|b\rangle .
\end{gathered}
$$


Using (2.34) and the fact that $|b\rangle$ is an $s_{0}$ eigenvector $s_{0}|b\rangle=(2 b / 3)|b\rangle$, this implies

$$
\sum_{\ell=0}^{k-1} s_{-\ell-1} \epsilon_{\ell+1-k-a(a+2 b) / 3}^{(a)}|b\rangle=\frac{k}{a} \epsilon_{-k-a(a+2 b) / 3}^{(a)}|b\rangle .
$$

If we define the polynomial $P_{k}^{(a)}(s)$ by

$$
\epsilon_{-k-a(a+2 b) / 3}^{(a)}|b\rangle=P_{k}^{(a)}(s)|a+b\rangle
$$

then (2.36) becomes the recursion relation

$$
P_{k}^{(a)}=\left(\frac{a}{k}\right) \sum_{\ell=0}^{k-1} s_{-\ell-1} P_{k-1-\ell}^{(a)}
$$

The initial condition for this recursion, $P_{0}^{(a)}=1$, is provided by (2.34). The first few solutions are $P_{1}^{(a)}=a s_{-1}$ and $P_{2}^{(a)}=\frac{a}{2}\left(s_{-2}+a s_{-1} s_{-1}\right)$.

To conclude this discussion of the $\mathbf{Z}_{4}$ parafermion Fock space, we write down all the low-lying states, since they will be useful for building the low-lying FSS physical states. Consider first the states in the parafermion sectors with $S U(2)$ quantum number $m=0$. This is the sector analogous to the superstring Neveu-Schwarz sector - it consists of all states generated from the vacuum by modes of the energy operators $\epsilon^{( \pm 1)}$. The complete set of independent states for the lowest levels of the $m=0$ sector can be written as follows:

$$
\begin{array}{cc}
|0\rangle & \text { level } 0, \\
\epsilon_{-1 / 3}^{(1)}|0\rangle, \epsilon_{-1 / 3}^{(-1)}|0\rangle & \text { level } \frac{1}{3}, \\
s_{-1}|0\rangle & \text { level } 1, \\
\epsilon_{-4 / 3}^{(2)}|0\rangle, \epsilon_{-4 / 3}^{(-2)}|0\rangle, s_{-1} \epsilon_{-1 / 3}^{(1)}|0\rangle, s_{-1} \epsilon_{-1 / 3}^{(-1)}|0\rangle & \text { level } \frac{4}{3},
\end{array}
$$

The counting of states here agrees with that implied by the characters $\mathcal{Z}_{0}^{0}+\mathcal{Z}_{0}^{2}$ and $\mathcal{Z}_{0}^{1}$ obtained previously. Note that the GCR's given in eq. (2.27) have been used to eliminate states that are dependent on the ones listed. Indeed, it is easy to solve the recursion relations (2.37) and 2.38) to the first few levels to obtain the identities

$$
\begin{aligned}
& \epsilon_{-2 / 3}^{( \pm 1)} \epsilon_{-1 / 3}^{(\mp 1)}|0\rangle= \pm s_{-1}|0\rangle, \\
& s_{-1} \epsilon_{-1 / 3}^{( \pm 1)}|0\rangle= \pm \epsilon_{-4 / 3}^{( \pm 1)}|0\rangle,
\end{aligned}
$$

and

$$
\epsilon_{-1}^{( \pm 1)} \epsilon_{-1 / 3}^{( \pm 1)}|0\rangle=\epsilon_{-4 / 3}^{( \pm 2)}|0\rangle
$$


Similarly, the analogs of the Ramond sector are the $m= \pm 1$ sectors built up by $\epsilon^{( \pm 1)}$ modes acting on the $\epsilon^{( \pm 1 / 2)}$ spin fields. The complete set of independent states for the lowest levels are

$$
\begin{array}{cc}
\epsilon_{-1 / 12}^{(1 / 2)}|0\rangle, \epsilon_{-1 / 12}^{(-1 / 2)}|0\rangle & \text { level } \frac{1}{12}, \\
\epsilon_{-3 / 4}^{(3 / 2)}|0\rangle, \epsilon_{-3 / 4}^{(-3 / 2)}|0\rangle & \text { level } \frac{1}{12}+\frac{2}{3}, \\
s_{-1} \epsilon_{-1 / 12}^{(1 / 2)}|0\rangle, s_{-1} \epsilon_{-1 / 12}^{(-1 / 2)}|0\rangle & \text { level } \frac{1}{12}+1, \\
\ldots & \ldots
\end{array}
$$

These states can be written in other ways using the identities following from the GCRs:

$$
\begin{gathered}
\epsilon_{0}^{(\mp 1)} \epsilon_{-1 / 12}^{( \pm 1 / 2)}|0\rangle=\epsilon_{-1 / 12}^{(\mp 1 / 2)}|0\rangle, \\
\epsilon_{-2 / 3}^{( \pm 1)} \epsilon_{-1 / 12}^{( \pm 1 / 2)}|0\rangle=\epsilon_{-3 / 4}^{( \pm 3 / 2)}|0\rangle, \\
\epsilon_{-1 / 3}^{(\mp 1)} \epsilon_{-3 / 4}^{( \pm 3 / 2)}|0\rangle=\mp s_{-1} \epsilon_{-1 / 12}^{( \pm 1 / 2)}|0\rangle,
\end{gathered}
$$

and

$$
\epsilon_{-1}^{(\mp 1)} \epsilon_{-1 / 12}^{( \pm 1 / 2)}|0\rangle=\mp s_{-1} \epsilon_{-1 / 12}^{(\mp 1 / 2)}|0\rangle .
$$

Note also that if we had only used the standard commutator (2.28) as the defining commutation relation, rather than the GCR (2.29), we would not have found the correct counting of states. For example, the relation (2.41) would have been absent.

\subsection{The coordinate boson field}

To complete the description of one dimension's worth of the FSS Fock space, we must tensor the parafermion theory with a free boson $X$, which will have the interpretation of a space-time coordinate field. We will only consider the left-moving (holomorphic) part of this boson on the world-sheet. We set its normalization by

$$
\langle X(z) X(w)\rangle=-\ln (z-w),
$$

from which follows the energy-momentum tensor

$$
T_{X}(z)=-\frac{1}{2}:(\partial X(z))^{2}: .
$$

The primary fields are

$$
V_{p}(z) \equiv: \mathrm{e}^{i p X(z)}:
$$

of dimension $\Delta\left(V_{p}\right)=p^{2} / 2$. Because $X$ is not compactified, the momentum $p$ can take on any real value. The mode expansion of $X$ is

$$
X(z)=x_{0}-i \alpha_{0} \ln (z)+i \sum_{n \neq 0} \frac{1}{n} \alpha_{n} z^{-n},
$$


where the modes satisfy the standard commutation relations

$$
\left[x_{0}, \alpha_{0}\right]=i, \quad\left[\alpha_{n}, \alpha_{m}\right]=n \delta_{n+m} .
$$

The Fock space is built up by these modes from the vacuum $|0\rangle$ satisfying $\alpha_{n}|0\rangle=0$ for $n \geq 0$. The highest weight states

$$
|p\rangle \equiv V_{p}(0)|0\rangle=\mathrm{e}^{i p x_{0}}|0\rangle
$$

satisfy $\alpha_{0}|p\rangle=p|p\rangle$ and $\alpha_{n}|p\rangle=0$ for $n>0$.

Upon tensoring the $\mathbf{Z}_{4}$ parafermion theory with the coordinate boson, we obtain a CFT with central charge $c_{0}=2$, and energy-momentum tensor

$$
T(z)=T_{X}(z)+T_{\rho}(z)
$$

Defining the Virasoro modes in the usual way by $T(z)=\sum z^{-n-2} L_{n}$, we find

$$
L_{n}=\frac{1}{2} \sum_{\ell}: \alpha_{\ell} \alpha_{n-\ell}:+\frac{3}{4} \sum_{\ell}: s_{\ell} s_{n-\ell}: .
$$

Note that $T(z)$ is not the energy-momentum tensor for the full FSS, but instead corresponds to only one dimension of the FSS. We take the full $K=4$ FSS Fock space to be the tensor product of $D$ copies of this $c_{0}=2 \mathrm{CFT}$, where $D$ is the number of space-time dimensions. The full $L_{n}$ 's will then obey a Virasoro algebra with central charge $c=D c_{0}$. In Sect. 3 we will derive expressions for the fractional supercurrent $G(z)$ in the $c_{0}=2$ and $c=D c_{0}$ Fock spaces.

The $\mathbf{Z}_{4}$ parafermion fields $\epsilon^{(a)}(z)$ and the coordinate boson field $\partial X(z)$ are primary fields with respect to $T(z)$ with conformal dimensions $a^{2} / 3$ and 1 , respectively. Their modes satisfy the usual comutation relations with the $L_{n}$ :

$$
\begin{aligned}
{\left[L_{n}, \alpha_{m}\right] } & =-m \alpha_{n+m} \\
{\left[L_{n}, \epsilon_{r}^{(a)}\right] } & =\left[\left(\frac{a^{2}}{3}-1\right) n-r\right] \epsilon_{n+r}^{(a)} .
\end{aligned}
$$

\section{The fractional superconformal algebra}

In this section we construct the fractional supercurrent, $G(z)$, of the $K=4$ FSS. This current is the analog of the dimension- $3 / 2$ supercurrent of the superstring. We argue that it is a dimension- $4 / 3$ chiral primary field in the CFT describing the FSS, and find its explicit form in the free boson representation introduced in Sect. 2. This current $G(z)$ and the energy-momentum tensor $T(z)$ together generate the fractional superconformal algebra. By analogy with the superconformal gauge of the superstring, in which the energy-momentum tensor and supercurrent generate the 
physical state conditions, we discuss the physical state conditions that follow from the fractional superconformal mode algebra.

It is important to note that the real justification for singling out the dimension$4 / 3$ field as the fractional supercurrent, and for taking its modes as generators of FSS physical state conditions, rests in showing that a sensible spectrum results. In Sects. 1 and 5 we will succeed in doing this for the massless states in the spectrum, but will fail for the massive states.

\subsection{Constructing the fractional supercurrent}

We start by constructing the fractional supercurrent in the CFT corresponding to a single dimension of the $K=4$ FSS. Later we will tensor together $D$ components to get an expression for the full fractional supercurrent.

Recall the form of the supercurrent in a single dimension of the usual superstring:

$$
G_{s}=\psi \partial X
$$

In terms of a $\mathbf{Z}_{2}$ parafermion description, the Majorana fermion field $\psi$ is the Virasoro primary field in the $j=1, m=0$ parafermion sector $\left[\phi_{0}^{1}\right]$. By analogy with this construction, we might naively expect the fractional supercurrent to have the form $G \sim \phi_{0}^{1} \partial X$, where $\phi_{0}^{1}$ stands for a Virasoro primary in the $\left[\phi_{0}^{1}\right]$ sector of the $\mathbf{Z}_{4}$ parafermion theory. However, as we have seen in Sect. 2, the $\mathbf{Z}_{4}$ parafermion sectors (unlike the $\mathbf{Z}_{2}$ case) contain an infinite number of primary fields. In particular the $\left[\phi_{0}^{1}\right]$ sector consists of a tower of primary fields $\epsilon^{(3 n \pm 1)}$ of dimensions $(3 n \pm 1)^{2} / 3$ for $n \in \mathbf{Z}$. The lowest-dimension fields are the so-called energy operators $\epsilon^{( \pm 1)}$ with dimension $1 / 3$, implying the fractional supercurrent has dimension $4 / 3$. Note, however, that there are also Virasoro primary fields $\epsilon^{( \pm 2)}$ in the $\left[\phi_{0}^{1}\right]$ sector with dimension $4 / 3$. In general, if we demand that the algebra generated by the fractional supercurrent and the energy-momentum tensor close, we will find that both the $\epsilon^{( \pm 1)}$ and $\epsilon^{( \pm 2)}$ fields will have to be included in the definition of $G$.

Another difference between the fractional supercurrent and the usual supercurrent (3.1) stems from the fact that the $\left[\phi_{0}^{1}\right]$ sector appears with multiplicity two in the free boson representation of the $\mathbf{Z}_{4}$ parafermion theory described in Sect. 2. This means that the fractional supercurrent $G$ will be naturally split into two currents, $G^{+}(z)$ and $G^{-}(z)$. Indeed, demanding that two spin- $4 / 3$ currents and the energymomentum tensor built from a boson field $X$ and $\mathbf{Z}_{4}$ parafermion fields form a closed operator product algebra, one discovers the expressions

$$
\begin{aligned}
G^{+}(z) & =\frac{1}{\sqrt{2}} \epsilon^{(1)}(z) i \partial X(z)+\frac{1}{2} \epsilon^{(-2)}(z), \\
G^{-}(z) & =\frac{1}{\sqrt{2}} \epsilon^{(-1)}(z) i \partial X(z)+\frac{1}{2} \epsilon^{(2)}(z), \\
T(z) & =-\frac{1}{2}: \partial X(z) \partial X(z):-\frac{3}{4}: \partial \rho(z) \partial \rho(z):
\end{aligned}
$$


which satisfy the algebra

$$
\begin{aligned}
G^{+}(z) G^{+}(w) & =\frac{1}{(z-w)^{4 / 3}}\left\{G^{-}(w)+\frac{1}{2}(z-w) \partial G^{-}(w)\right\}, \\
G^{-}(z) G^{-}(w) & =\frac{1}{(z-w)^{4 / 3}}\left\{G^{+}(w)+\frac{1}{2}(z-w) \partial G^{+}(w)\right\}, \\
G^{+}(z) G^{-}(w) & =\frac{(1 / 2)}{(z-w)^{8 / 3}}\left\{\frac{3}{2}+2(z-w)^{2} T(w)\right\} .
\end{aligned}
$$

In the above OPEs, only the singular terms (i.e. those with negative powers of $z-w$ ) have been included. The normalization of the right hand side of the $G^{+} G^{-}$OPE does not completely fix the normalization of the $G^{ \pm}$currents separately. This extra freedom was used make the structure constants appearing in the $G^{+} G^{+}$and $G^{-} G^{-}$ OPEs equal. We have also fixed the trivial symmetry which takes all $\epsilon^{(a)}(z)$ to $x^{a} \epsilon^{(a)}(z)$ for $x$ some complex number, corresponding to a shift in the origin of the $\rho(z)$ boson.

This split algebra (3.3) is a special case of the spin- $4 / 3$ algebra studied by Zamolodchikov and Fateev [17]. An important property of the split algebra is that its currents, $G^{+}$and $G^{-}$, inherit definite $\mathbf{Z}_{3}$ charges, $q=1$ and -1 respectively, from the parafermion representation. This is reflected in the fact that $G^{ \pm}$satisfy abelian braiding relations. In terms of their OPEs, this means that only one kind of cut appears on the right hand side. For example, the terms in the $G^{+} G^{+}$OPE are all proportional to $(z-w)^{n+2 / 3}$ where $n$ in an integer. Because of this, we will be able to derive generalized commutation relations satisfied by the current modes, following the discussion in Appendix B.

The full fractional superconformal current $G(z)$ of the FSS is defined in the $D$ fold tensor product space of coordinate boson plus $\mathbf{Z}_{4}$ parafermion theories. Let us rename the currents in (3.2) associated with the $\mu$ th dimension $G^{(\mu) \pm}$ and $T^{(\mu)}$. Then the algebra generated by the fields

$$
\begin{aligned}
& G(z)=\sum_{\mu=0}^{D-1}\left(G^{(\mu)+}(z)+G^{(\mu)-}(z)\right), \\
& T(z)=\sum_{\mu=0}^{D-1} T^{(\mu)}(z),
\end{aligned}
$$

is the full fractional superconformal algebra:

$$
\begin{aligned}
T(z) T(w) & =\frac{D}{(z-w)^{4}}+\frac{2 T(w)}{(z-w)^{2}}+\frac{\partial T(w)}{(z-w)}, \\
T(z) G(w) & =\frac{\frac{4}{3} G(w)}{(z-w)^{2}}+\frac{\partial G(w)}{(z-w)}, \\
G(z) G(w) & =\frac{1}{(z-w)^{8 / 3}}\left\{\frac{3 D}{2}+2(z-w)^{2} T(w)\right\}
\end{aligned}
$$




$$
+\frac{1}{(z-w)^{4 / 3}}\left\{G(w)+\frac{1}{2}(z-w) \partial G(w)\right\} .
$$

It is this non-local, non-abelianly braided algebra which we will take as the generator of the physical state conditions for the $K=4 \mathrm{FSS}$. It will play a role in the FSS analogous to that played by the superVirasoro algebra in the superstring. The representation theory of the fractional superconformal algebras (3.3) and (3.5) is similar to that of the superVirasoro algebra in that both have a discrete series of minimal unitary representations. However, the fractional superconformal algebras have some qualitatively new properties arising from their non-local nature (the cuts in their OPEs). The representation theory of the fractional superconformal algebras is discussed further in Appendix Q.

\subsection{Fractional superconformal mode algebra}

We now turn to the mode expansions and generalized commutators following from the fractional superconformal algebra. We noted above that the split algebra (3.3) has a $\mathbf{Z}_{3}$-symmetry and is abelianly braided. Therefore the arguments of Appendix B can be directly applied in deriving the GCRs following from the split algebra. The mode expansions for the full superconformal current can be built from the split algebra pieces.

The Fock space of split algebra representations fall into sectors $\mathcal{H}_{q}$ labelled by their $\mathbf{Z}_{3}$ charge. The currents $G^{+}$and $G^{-}$have $\mathbf{Z}_{3}$ charges $q=+1$ and $q=-1$, respectively, and act on the Fock space sectors according to the rules

$$
G^{+}: \mathcal{H}_{q} \rightarrow \mathcal{H}_{q+1}, \quad G^{-}: \mathcal{H}_{q} \rightarrow \mathcal{H}_{q-1}
$$

where the $\mathbf{Z}_{3}$ charge is defined mod 3. With these actions, the mode expansions of $G^{+}$and $G^{-}$are defined as

$$
\begin{aligned}
G^{+}(z) \chi_{q}(0) & =\sum_{n} z^{n-q / 3} G_{-1-n-(1-q) / 3}^{+} \chi_{q}(0) \\
G^{-}(z) \chi_{q}(0) & =\sum_{n} z^{n+q / 3} G_{-1-n-(1+q) / 3}^{-} \chi_{q}(0)
\end{aligned}
$$

where $\chi_{q}$ is an arbitrary state in $\mathcal{H}_{q}$. These mode expansions can be inverted to give

$$
\begin{aligned}
G_{n-(1-q) / 3}^{+} \chi_{q}(0) & =\oint_{\gamma} \frac{\mathrm{d} z}{2 \pi i} z^{n+q / 3} G^{+}(z) \chi_{q}(0) \\
G_{n-(1+q) / 3}^{-} \chi_{q}(0) & =\oint_{\gamma} \frac{\mathrm{d} z}{2 \pi i} z^{n-q / 3} G^{-}(z) \chi_{q}(0) .
\end{aligned}
$$

Here, $\gamma$ is a contour encircling the origin once, where $\chi_{q}(0)$ is inserted.

From the argument reviewed in Appendix B, the GCRs for the current modes of the split algebra (3.3) can be derived. As explained in Appendix B, there are many 
GCRs that can be derived from a single OPE, depending on how many terms on the right hand side of the OPE one wishes to include. We will include only the singular terms, shown in eq. (3.3). With this choice, the split algebra GCRs become [17]

$$
\begin{gathered}
\sum_{\ell=0}^{\infty} c_{\ell}^{(-2 / 3)}\left[G_{\frac{q}{3}+n-\ell}^{+} G_{\frac{2+q}{3}+m+\ell}^{+}-G_{\frac{q}{3}+m-\ell}^{+} G_{\frac{2+q}{3}+n+\ell}^{+}\right]= \\
\frac{1}{2}(n-m) G_{\frac{2+2 q}{3}+n+m}^{-}, \\
\sum_{\ell=0}^{\infty} c_{\ell}^{(-2 / 3)}\left[G_{-\frac{q}{3}+n-\ell}^{-} G_{\frac{2-q}{3}+m+\ell}^{-}-G_{-\frac{q}{3}+m-\ell}^{-} G_{\frac{2-q}{3}+n+\ell}^{-}\right]= \\
\frac{1}{2}(n-m) G_{\frac{2-2 q}{3}+n+m}^{+}, \\
\sum_{\ell=0}^{\infty} c_{\ell}^{(-1 / 3)}\left[G_{\frac{1+q}{3}+n-\ell}^{+} G_{-\frac{1+q}{3}+m+\ell}^{-}+G_{-\frac{2+q}{3}+m-\ell}^{-} G_{\frac{2+q}{3}+n+\ell}^{+}\right]= \\
L_{n+m}+\frac{3}{8}\left(n+1+\frac{q}{3}\right)\left(n+\frac{q}{3}\right) \delta_{n+m},
\end{gathered}
$$

where these expressions are understood to be acting on a state in $\mathcal{H}_{q}$. For completeness, we also write down the Virasoro algebra and the standard commutators following from the fact that $G^{ \pm}$are dimension $4 / 3$ primary fields:

$$
\begin{aligned}
{\left[L_{m}, L_{n}\right] } & =(m-n) L_{m+n}+\frac{1}{6}\left(m^{3}-m\right) \delta_{m+n} \\
{\left[L_{m}, G_{r}^{ \pm}\right] } & =\left(\frac{1}{3} m-r\right) G_{m+r}^{ \pm}
\end{aligned}
$$

where the moding $r$ is the one appropriate to whichever $\mathbf{Z}_{3}$ sector the $G^{ \pm}$currents are acting on. The Virasoro algebra has central charge $c_{0}=2$, corresponding to one dimension of the full $K=4 \mathrm{FSS}$.

It is useful to have expressions for the $G^{ \pm}$modes in terms of the $\mathbf{Z}_{4}$ parafermion and coordinate boson modes that we used in Sect. 2 to construct the Fock space of FSS states. Using the explicit form for the currents (3.2) and the parafermion and boson mode expansions (2.25) and (2.51), we express the current modes as

$$
\begin{aligned}
G_{n-(1-q) / 3}^{+} & =\frac{1}{\sqrt{2}} \sum_{\ell \in \mathbf{Z}} \alpha_{-\ell} \epsilon_{n+\ell-(1-q) / 3}^{(1)}+\frac{1}{2} \epsilon_{n-(1-q) / 3}^{(-2)}, \\
G_{n-(1+q) / 3}^{-} & =\frac{1}{\sqrt{2}} \sum_{\ell \in \mathbf{Z}} \alpha_{-\ell} \epsilon_{n+\ell-(1+q) / 3}^{(-1)}+\frac{1}{2} \epsilon_{n-(1+q) / 3}^{(2)} .
\end{aligned}
$$

The analogous formula for the energy-momentum modes $L_{n}$ has already been given in eq. (2.55). From these expressions and the parafermion and boson commutation relations derived in Sect. 2, one can verify the current commutators (3.9) and (3.10).

The $G(z)$ current of the full fractional superconformal algebra (3.5) is a sum of one copy of $G^{+}$and $G^{-}$for each space-time dimension. The full Fock space can 
be decomposed into $\mathbf{Z}_{3}$ sectors for each dimension, which we denote by $\mathcal{H}_{\left\{q_{\mu}\right\}}$ where $\mu=0,1, \ldots, D-1$, and $D$ is the space-time dimension. Since the action of $G$ mixes these sectors,

$$
G: \mathcal{H}_{\left\{q_{0}, \ldots, q_{D-1}\right\}} \rightarrow \bigoplus_{\mu=0}^{D-1}\left(\mathcal{H}_{\left\{q_{0}, \ldots, q_{\mu}-1, \ldots, q_{D-1}\right\}} \oplus \mathcal{H}_{\left\{q_{0}, \ldots, q_{\mu}+1, \ldots, q_{D-1}\right\}}\right)
$$

it will have no definite moding when acting on states in these sectors. However, we can define mode operators for $G(z)$ acting between two specific sectors:

$$
G_{r}^{(\mu) \pm}: \mathcal{H}_{\left\{q_{0}, \ldots, q_{\mu}, \ldots, q_{D-1}\right\}} \rightarrow \mathcal{H}_{\left\{q_{0}, \ldots, q_{\mu} \pm 1, \ldots, q_{D-1}\right\}} .
$$

The moding $r$ is determined (up to an integer part) by the initial and final sectors. In other words, because of its nonabelian braiding, the moding of the currents depends not only on the Fock space sector upon which it is acting, but also on the sector it maps to. The mode expansion of $G(z)$ can thus be written

$$
G(z)=\sum_{\mu=0}^{D-1} \sum_{n \in \mathbf{Z}}\left(z^{n-q_{\mu} / 3} G_{-1-n-\left(1-q_{\mu}\right) / 3}^{(\mu)+}+z^{n+q_{\mu} / 3} G_{-1-n-\left(1+q_{\mu}\right) / 3}^{(\mu)-}\right),
$$

when acting on an arbitrary state in $\mathcal{H}_{\left\{q_{\mu}\right\}}$. From our construction of the fractional supercurrent from the split algebra currents (3.4), it is clear that the modes $G^{(\mu) \pm}$ are just $G^{ \pm}$for the $\mu$ th $\mathbf{Z}_{4}$ parafermion plus coordinate boson CFT in the full $D$-fold tensor product CFT of the FSS.

When we apply the physical state conditions, we will be interested in acting with a given moding of the full current $G(z)$ on states which are not in a definite Fock space sector. We can define such a mode operator as follows. Write the Fock space states as a $3^{D}$-dimensional column vector with elements labeled by the set $\left\{q_{\mu}\right\}$ of their $\mathbf{Z}_{3}$ quantum numbers in each space-time dimension. Then the action of $G(z)$ on this state can be represented by a $3^{D} \times 3^{D}$ matrix of operators with $G^{(\mu) \pm}$ being its various (off-diagonal) elements. We can think of the coordinate boson and $\mathbf{Z}_{4}$ parafermion modes as matrices in a similar way. Thus, if, for example, there were only one space-time dimension, the $\epsilon_{n-(1-q) / 3}^{(1)}$ mode would be represented by a $3 \times 3$ matrix with one non-zero element, namely the mode itself, in column $\{q\}$ and row $\{q+1\}$. For $D$ dimensions, $\epsilon_{n-(1-q) / 3}^{(1), \mu}$ would be the tensor product of $D 3 \times 3$ matrices

$$
\epsilon_{n-(1-q) / 3}^{(1), \mu}=\mathbb{1} \otimes \ldots \otimes \epsilon_{n-(1-q) / 3}^{(1)} \otimes \ldots \otimes \mathbb{1},
$$

where the $\epsilon^{(1)}$-matrix is in the $\mu$ th position. Similar comments apply to the general parafermion mode $\epsilon_{r}^{(a), \mu}$ and the coordinate boson modes $\alpha_{n}^{\mu}$. With this convention, we can write the general formula for the mode $G_{r}$ in terms of boson and parafermion modes from the expressions (3.11):

$$
G_{r}=\sum_{\mu=0}^{D-1}\left(\frac{1}{\sqrt{2}} \sum_{\ell \in \mathbf{Z}}\left(\alpha_{-\ell}\right)_{\mu}\left[\epsilon_{r+\ell}^{(1), \mu}+\epsilon_{r+\ell}^{(-1), \mu}\right]+\frac{1}{2}\left[\epsilon_{r}^{(-2),(\mu)}+\epsilon_{r}^{(2),(\mu)}\right]\right),
$$


for an arbitrary moding $r \in \mathbf{Z} / 3$. The matrix notation we have introduced to write (3.16) may be more easily utilized by noting that it is formally equivalent to adopting the convention that when a parafermion mode operator has the wrong moding to act on a given sector, it vanishes.

Note that we have embellished the boson and parafermion modes with an extra superscript $\mu$ denoting which of the $D$ tensored copies they act in. In the case of the coordinate boson modes $\alpha^{\mu}$, we interpret this superscript as a space-time Lorentz covariant index. Lorentz invariance of the fractional supercurrent then implies that the index on the $\epsilon^{( \pm 1), \mu}$ fields is also Lorentz covariant, and that a factor of the Minkowski metric should be understood in the first term of (3.16). By the same token, the index on the $\epsilon^{( \pm 2),(\mu)}$ fields can not be Lorentz covariant. This should not be surprising, since, by the parafermion GCR $(2.27)$ any $\epsilon^{( \pm 2)}$ mode can be written in terms of two $\epsilon^{( \pm 1)}$ modes; for example

$$
\begin{array}{r}
\epsilon_{n+m+(2 q-1) / 3}^{(2),(\mu)}=\sum_{\ell=0}^{\infty} c_{\ell}^{(-5 / 3)}\left[\epsilon_{n-1-\ell+q / 3}^{(1), \mu} \epsilon_{m+1+\ell+(q-1) / 3}^{(1), \mu}\right. \\
\left.+\epsilon_{m-1-\ell+q / 3}^{(1), \mu} \epsilon_{n+1+\ell+(q-1) / 3}^{(1), \mu}\right]
\end{array}
$$

Replacing the $\sum_{\mu} \epsilon_{r}^{( \pm 2),(\mu)}$ terms in (3.16) with the above expression (and including a factor of the Minkowski metric to tie the indices together), we recover a space-time Lorentz covariant interpretation of the index $\mu$.

The $G_{r}$ modes (3.16) of the full fractional supercurrent satisfy no simple GCRs such as those satisfied by the component modes $G_{r}^{(\mu) \pm}$ in (3.9). The reason for this is that for $\mu \neq \nu, G_{r}^{(\mu) \pm}$ and $G_{s}^{(\nu) \pm}$ satisfy simple (anti)commutation relations which can not be combined with the GCRs in (3.9) to form expressions involving only the full $G_{r}$ modes. This is simply a reflection of the fact that the $G^{(\mu) \pm}(z)$ currents satisfy abelian braid relations, whereas the full current $G(z)$, does not.

The energy-momentum tensor and its mode expansion for the full $D$-dimensional FSS is built up in a similar manner from pieces acting between Fock space sectors. However, since the $L_{n}$ moding is always integral (equivalently, the $L_{n}$ modes act diagonally on the Fock space sectors), there is never any need to keep track of the sector indices $q$ and $\mu$. So, in the matrix notation, these modes satisfy the usual commutators

$$
\begin{aligned}
& {\left[L_{m}, L_{n}\right]=(m-n) L_{m+n}+\frac{D}{6}\left(m^{3}-m\right) \delta_{m+n}} \\
& {\left[L_{m}, G_{r}\right]=\left(\frac{m}{3}-r\right) G_{m+r} .}
\end{aligned}
$$

\subsection{Physical state conditions}

In the usual superstring, the physical state conditions are constraints following from gauge-fixing the local world-sheet symmetry. Classically these constraints in 
the superconformal gauge are given by the vanishing of the energy-momentum tensor and superconformal current: $T(z)=G_{s}(z)=0$. The full local world-sheet symmetry of the $K=4$ FSS is unknown, though it should include reparametrization and Weyl invariance. We will assume that some analog of the superconformal gauge exists in the FSS, giving rise to an algebra of constraints generated by the vanishing of $T(z)$ and the fractional superconformal current $G(z)$. In other words, we assume that the fractional superconformal algebra is the quantum version of some classical constraint algebra. Thus, although we do not know of any classical local symmetry on the world-sheet that gives rise to a spin- $4 / 3$ current as a constraint upon gauge-fixing, we nevertheless assume the weak physical state conditions

$$
\langle\psi|: T(z):| \phi\rangle=\langle\psi|: G(z):| \phi\rangle=0
$$

for any physical states $|\phi\rangle$ and $|\psi\rangle$. The normal ordering symbols are there to remind us that there may be normal ordering constants in the quantum definition of the currents.

The energy-momentum constraint is "factorized" by expressing $T(z)$ in terms of its mode operators,

$$
T(z)=\sum_{n} L_{n} z^{-n-2}
$$

and using the mode algebra following from the constraint algebra OPEs

$$
\left[L_{m}, L_{n}\right]=(m-n) L_{m+n}+\frac{D}{6}\left(m^{3}-m\right) \delta_{m+n},
$$

as well as the hermiticity conditions

$$
\left(L_{n}\right)^{\dagger}=L_{-n},
$$

to factorize the quantum constraints into the usual physical state conditions

$$
\begin{aligned}
& L_{0}|\phi\rangle=v|\phi\rangle, \\
& L_{n}|\phi\rangle=0, \quad n \geq 0 .
\end{aligned}
$$

Here $v$ is the intercept, a normal ordering constant in the definition of $T$. This is a consistent set of constraints because the positive modes form a closed subalgebra of the Virasoro algebra generated by the two modes $L_{1}$ and $L_{2}$.

Let us mimic this discussion in the case of the fractional superconformal constraint. In our matrix notation, between two physical states, $G(z)$ will have the mode expansion

$$
\langle\psi|: G(z):| \phi\rangle=\sum_{r \in \mathbf{Z} / 3} z^{r-4 / 3}\left\langle\psi\left|: G_{r}:\right| \phi\right\rangle
$$

From the explicit formula (3.16) for the current modes and the hermiticity properties of the $\mathbf{Z}_{4}$ parafermion field modes

$$
\left(\epsilon_{r}^{(a)}\right)^{\dagger}=\epsilon_{-r}^{(-a)}
$$


it follows that the fractional superconformal current satisfies the hermiticity condition

$$
\left(G_{r}\right)^{\dagger}=G_{-r} .
$$

This makes it plausible to take as physical state conditions factorizing the $G(z)$ quantum constraint (3.19)

$$
\begin{aligned}
& G_{0}|\phi\rangle=\beta|\phi\rangle, \\
& G_{r}|\phi\rangle=0, \quad r>0,
\end{aligned}
$$

where $\beta$ is an undetermined normal-ordering constant.

From the GCRs for the fractional supercurrent components (3.9), it is not hard to see that the physical state conditions (3.27) are consistent with themselves and with the Virasoro conditions (3.23). In particular, from (3.9) and (3.18), it is not possible to derive an identity of the form

$$
G_{-r} G_{s}|\phi\rangle=G_{s-r}|\phi\rangle
$$

for $r>s>0$, or similar relations with $L_{-r}$ replacing the $G_{-r}$ mode. Because of the infinite sums that appear in the GCR algebra, it is unclear in what sense, if any, the positive modes of $G$ can be said to form a closed subalgebra of the constraint algebra. However, from the $L_{m}-G_{r}$ commutator (3.18), we can generate all the conditions (3.23) and (3.27) from the set $\left\{L_{0}, L_{1}, G_{0}, G_{1 / 3}, G_{2 / 3}\right\}$.

\section{Low-lying states of the fermionic sector}

In this section we construct the full space of states in the fermionic sector of a $D$ dimensional open $K=4$ FSS. The ground state of this sector forms a representation of the $D$-dimensional Clifford algebra. We then solve for the subset of states at the massless level that satisfy the physical state conditions. They obey Lorentz covariant equations of motion as well as a sufficient number of constraints to eliminate all unphysical degrees of freedom. The number of propagating modes at these levels can be halved by a chirality projection analogous to the GSO projection [16] in the Ramond sector of the superstring. We derive, using a counting argument involving the Euler pentagonal number theorem, the explicit form of the chirality operator at all levels of the fermionic sector.

\subsection{Fermionic ground state and Clifford algebra}

In Sect. 2 and Appendix D we have argued that the fermionic sector of the FSS Fock space consists of all states obtained by successive applications of the dimension$1 / 3 \epsilon^{( \pm 1), \mu}(z)$ parafermion fields and the coordinate boson fields $\partial X^{\mu}(z)$ on the ground state:

$$
|\alpha, p\rangle \equiv\left(\prod_{\mu=0}^{D-1} \epsilon^{( \pm 1 / 2), \mu}\right): \mathrm{e}^{i p \cdot X}:|0\rangle .
$$


Here $\epsilon^{( \pm 1 / 2), \mu}(z)$ are the two dimension-1/12 parafermion spin fields associated with the $\mu$ th space-time dimension. At a given momentum $p$, the $\alpha$ index of the ground state labels its $2^{D}$-fold degeneracy. We will show that the zero-modes of the $\epsilon^{( \pm 1), \mu}$ fields naturally form the $D$-dimensional Clifford algebra when acting on (4.1).

Let us start by considering the CFT corresponding to a single space-time dimension. For the sake of notational simplicity, we rename the dimension-1/3 parafermion fields

$$
\epsilon \equiv \epsilon^{(+1)}, \quad \epsilon^{\dagger} \equiv \epsilon^{(-1)},
$$

and the spin states

$$
\begin{aligned}
& |+\rangle \equiv \epsilon_{-1 / 12}^{(+1 / 2)}|0\rangle, \\
& |-\rangle \equiv \epsilon_{-1 / 12}^{(-1 / 2)}|0\rangle .
\end{aligned}
$$

Note that the states $| \pm\rangle$ have $\mathbf{Z}_{3}$ charge $q=\mp 1$.

From the discussion of Sect. 2, the allowed modings $r$ of $\epsilon$ and $\epsilon^{\dagger}$ when acting on $| \pm\rangle$ are either $r=n$ or $r=n-2 / 3$, where $n \in \mathbf{Z}$. The integral modings map between states with $\mathbf{Z}_{3}$ charge $q=+1$ and $q=-1$, whereas the other modings map between $q= \pm 1$ and $q=0$ states. In particular, by eq. (2.44) we find that

$$
\begin{aligned}
\epsilon_{0}|+\rangle & =|-\rangle, \\
\epsilon_{0}^{\dagger}|-\rangle & =|+\rangle .
\end{aligned}
$$

Fig. 1 summarizes the allowed modings of the integral winding-number fields $\epsilon^{(a)}$, $a \in \mathbf{Z}$, on the different $\mathbf{Z}_{3}$ sectors.

We can think of $| \pm\rangle$ as basis vectors in a two-dimensional space of ground states $|\alpha\rangle, \alpha \in\{1,2\}$ :

$$
|1\rangle \equiv\left(\begin{array}{c}
|+\rangle \\
0
\end{array}\right), \quad|2\rangle \equiv\left(\begin{array}{c}
0 \\
|-\rangle
\end{array}\right) .
$$

Acting on this space we can define the operators $\tilde{\epsilon}_{n}$ in the following way:

$$
\tilde{\epsilon}_{n} \equiv\left(\begin{array}{cc}
0 & \epsilon^{\dagger} \\
\epsilon_{n} & 0
\end{array}\right), \quad n \in \mathbf{Z}
$$

(In terms of the matrix notation for the parafermion modes described in Sect. 3, (4.6) is simply the statement $\tilde{\epsilon}_{n}=\epsilon_{n}+\epsilon_{n}^{\dagger}$.) From (4.4) we learn

$$
\tilde{\epsilon}_{0} \tilde{\epsilon}_{0}|\alpha\rangle=|\alpha\rangle
$$

which can be rewritten as the one-dimensional Clifford algebra

$$
\left\{\tilde{\epsilon}_{0}, \tilde{\epsilon}_{0}\right\}|\alpha\rangle=2|\alpha\rangle
$$

Thus we can identify $\tilde{\epsilon}_{0}$ with the gamma matrix of the Clifford algebra when acting on the ground state $|\alpha\rangle$. 
Figure 1: The action and modings of the parafermion fields $\epsilon^{(a)}$ on the Fock space sectors of $\mathbf{Z}_{3}$ charge $q$. The parafermion field winding number (superscript) is understood mod 3, and its moding (subscript) mod 1.

Now we turn to the tensor product theory. In the $D$-dimensional tensor product theory, the (reducible) ground state of the fermionic sector is represented by

$$
|\alpha\rangle=\bigotimes_{\mu=0}^{D-1}\left|\alpha^{\mu}\right\rangle,
$$

where $\left|\alpha^{\mu}\right\rangle$ is the fermionic ground state of the $\mu$ th component of the tensor product theory and $\alpha=\left\{\alpha^{0}, \ldots, \alpha^{D-1}\right\}$. (Note that $\mu$ in these expressions is not a Lorentz index but simply a dimensional label.) $|\alpha\rangle$ spans a $2^{D}$-dimensional vector space. Ultimately we will reduce this space to obtain an irreducible spinor representation of the Lorentz group.

We define the $\tilde{\epsilon}_{n}^{\mu}$ modes acting on this space in the obvious way:

$$
\tilde{\epsilon}_{n}^{\mu} \equiv \mathbb{1} \otimes \ldots \otimes \tilde{\epsilon}_{n} \otimes \ldots \otimes \mathbb{1},
$$

where $\tilde{\epsilon}_{n}$ is in the $\mu$ th position in the tensor product. If we now consider the algebra of the $\tilde{\epsilon}_{0}^{\mu}$ modes, we find

$$
\left\{\tilde{\epsilon}_{0}^{\mu}, \tilde{\epsilon}_{0}^{\mu}\right\}|\alpha\rangle=2|\alpha\rangle .
$$

The modes $\tilde{\epsilon}_{0}^{\mu}$ and $\tilde{\epsilon}_{0}^{\nu}$ can be chosen to anticommute for $\mu \neq \nu$ by an appropriate choice of Klein factors [18]. Combining the $\mu=\nu$ and $\mu \neq \nu$ cases we find the $D$-dimensional Clifford algebra

$$
\left\{\tilde{\epsilon}_{0}^{\mu}, \tilde{\epsilon}_{0}^{\nu}\right\}|\alpha\rangle=2 g^{\mu \nu}|\alpha\rangle,
$$


so again we have, on the ground state,

$$
\tilde{\epsilon}_{0}^{\mu}|\alpha\rangle=\gamma^{\mu}|\alpha\rangle
$$

Here we take $g^{\mu \nu}$ to be the Minkowski metric. We can define $|\alpha\rangle$, the ground state of the fermionic sector in the tensor product theory, so that it furnishes an irreducible spinor representation of the Lorentz algebra $\mathrm{SO}(D-1,1)$. The dimension of this representation is $2^{D / 2}$. All of the states in this representation can be constructed from linear combinations of the states $|\alpha\rangle$ defined by eq. (4.9). This state must be multiplied by a momentum $p$ vertex operator made from the coordinate boson $X^{\mu}$ zero modes, to form the full fermionic sector ground state $|\alpha, p\rangle$.

It should be clear that so far this discussion closely parallels that of the Ramond sector of superstring theory. However, we must remark upon an unusual feature of the FSS Fock space. Because the $\tilde{\epsilon}^{\mu}$ modes satisfy GCRs instead of simple commutation or anticommutation relations, the Lorentz-covariant meaning of their space-time index $\mu$ is unclear. We have shown from the structure of the GCRs that the $\tilde{\epsilon}_{0}$ modes satisfy the Clifford algebra when acting on the ground state. However, this will not be true in general when they act upon excited states in the Fock space. Thus we are not free to replace the $\tilde{\epsilon}_{0}$ modes with gamma matrices unless they are acting on the ground state. More generally, by taking a tensor product structure for the FSS Fock space, we have only ensured a permutation symmetry among the different dimensions, but not necessarily the rotational symmetry of the Lorentz group.

\subsection{Massless physical states}

We now wish to obtain the set of physical states at the massless level. These states are a subset of the states spanned by the level 0 state $|\alpha, p\rangle$. As discussed in Sect. 3, physical states should satisfy the conditions (3.23) and (3.27):

$$
\begin{aligned}
L_{0}\left|\psi_{\text {phys }}\right\rangle & =v\left|\psi_{\text {phys }}\right\rangle, \\
G_{0}\left|\psi_{\text {phys }}\right\rangle & =\beta\left|\psi_{\text {phys }}\right\rangle, \\
L_{n}\left|\psi_{\text {phys }}\right\rangle & =0, \quad 0<n \in \mathbf{Z}, \\
G_{r}\left|\psi_{\text {phys }}\right\rangle & =0, \quad 0<r \in \mathbf{Z} / 3 .
\end{aligned}
$$

Here $v$ and $\beta$ are the as-yet-undetermined normal-ordering constants ("intercepts") of the fermionic sector.

Let us impose the physical state conditions (4.14) on level 0 states. Consider the general state at level 0 :

$$
\left|\psi_{0}\right\rangle=|\alpha, p\rangle u^{\alpha}(p)
$$

where $u^{\alpha}(p)$ is a spinor polarization. The $L_{r}$ and $G_{r}$ modes for $r>0$ automati-

cally annihilate $\left|\psi_{0}\right\rangle$, since it is the ground state of the fermionic sector. Thus the corresponding physical state conditions are identically satisfied. 
Because $|\alpha, p\rangle$ has conformal dimension $\frac{p^{2}}{2}+\frac{D}{12}$ for the $D$ space-time dimensional FSS Fock space, the $L_{0}$ physical state condition is equvalent to

$$
\left(L_{0}-v\right)\left|\psi_{0}\right\rangle=0 \Longrightarrow \frac{p^{2}}{2}+\frac{D}{12}=v,
$$

fixing the mass of the physical state in terms of the intercept $v$.

As follows easily from the expansion of the fractional supercurrent modes (3.16), the action of the $G_{0}$ mode on $\left|\psi_{0}\right\rangle$ is

$$
\begin{aligned}
G_{0}\left|\psi_{0}\right\rangle & =\frac{1}{\sqrt{2}}\left(\alpha_{0} \cdot \tilde{\epsilon}_{0}\right)|\alpha, p\rangle u^{\alpha} \\
& =|\alpha, p\rangle \not p u .
\end{aligned}
$$

Since $\tilde{\epsilon}_{0}$ acts on the ground state, we have replaced it with a $\gamma$-matrix. Thus the $G_{0}$ physical state condition is equivalent to

$$
\left(G_{0}-\beta\right)\left|\psi_{0}\right\rangle=0 \Longrightarrow \frac{1}{\sqrt{2}} \not p u(p)=\beta u(p)
$$

Comparing the $L_{0}$ and $G_{0}$ conditions shows that the two intercepts are related by

$$
\beta^{2}=v-\frac{D}{12}
$$

Since the $G_{0}$ mode, when acting on the ground state, is the simple product of an $X^{\mu}$ mode and a parafermion energy operator mode, it is hard to see why the normal-ordering constant $\beta$ should appear at all. (When the modes of the $\epsilon^{( \pm 2)}$ fields in the fractional supercurrent contribute, as can happen, we will see, in the bosonic sector, then $\beta$ could naturally be expected to be non-zero.) Setting $\beta=0$ implies that $v=D / 12$ and that the level zero physical states are massless. Indeed, this value of the intercept is the critical value, because a tower of extra null states appears in the FSS spectrum when $v=D / 12$. In particular, the fractional superconformal algebra (3.9) implies when acting on physical states with every space-time component in the ground state sector (i.e. with $\mathbf{Z}_{3}$ charge $q= \pm 1$ ), that

$$
G_{0} G_{0}=L_{0}-\frac{D}{12}
$$

Thus if $|\psi\rangle$ is a physical state with intercept $v=D / 12$, then $|\chi\rangle=G_{0}|\psi\rangle$ also obeys the physical state conditions with the same intercept. $|\chi\rangle$ is thus both physical and spurious, and therefore null. This whole argument is precisely analogous to the familiar argument for the Ramond ground state of the usual superstring.

* In the superstring, the intercept $v$ in the Ramond sector is usually set to zero by redefining the Virasoro mode $L_{0}$ to $L_{0}-D / 16$ so that the Ramond ground state automatically has $L_{0}$ eigenvalue zero. 
With the critical intercepts

$$
v=\frac{D}{12}, \quad \beta=0
$$

the $G_{0}$ condition (4.18) implies that $u^{\alpha}(p)$ satisfies the massless Dirac equation. For space-time dimension $D=6$, the number of complex degrees of freedom of the spinor representation is $2^{D / 2}=8$. Since the ground state is a massless spinor, and the space-time dimension is even, we can impose a Weyl condition, reducing this number to eight real degrees of freedom, of which only four propagate, since $u^{\alpha}(p)$ is the solution to a Dirac equation. This matches the counting of massless fermion propagating degrees of freedom found from the study of the partition function in Appendix D. The Weyl condition on the massless states is the analog of the GSO projection [16] in the Ramond sector of the superstring.

\subsection{Fermionic sector Fock space}

In order to consider higher-mass states in the fermionic sector, we will now derive a basis of states for all integral levels of the fermionic sector Fock space. We build the fermionic sector of the FSS Fock space by the action of the coordinate boson modes $\alpha_{n}^{\mu}$ and the parafermion energy operator modes $\epsilon_{r}^{( \pm 1)}$ on the ground state spinor $|\alpha, p\rangle$. In Sect. 2 we showed that in the single space-time component theory, the action of the energy operator modes on the spin field states $| \pm\rangle$ correspond to the parafermion sectors $\left[\phi_{ \pm 1}^{1}\right]$ and $\left[\phi_{ \pm 1}^{0}\right]$. Recall that $\left[\phi_{ \pm 1}^{1}\right]$ consists of the set of fields $\epsilon^{(3 n \pm 1 / 2)}$ of conformal dimensions $\mathbf{Z}+1 / 12$ and $\mathbf{Z}_{3}$ charge \pm 1 , whereas $\left[\phi_{ \pm 1}^{0}\right]$ consists of the $\epsilon^{(3 n+3 / 2)}$ fields of dimension $\mathbf{Z}+3 / 4$ and $\mathbf{Z}_{3}$ charge zero. We will call $\left[\phi_{ \pm 1}^{1}\right]$ the ground state sector, since it includes the ground state (4.1). The states in $\left[\phi_{ \pm 1}^{0}\right]$ we will refer to as "projection sector" states for reasons to be made clear later. From the moding rules summarized in Fig. 1, we see that we can define an operator, $\tilde{\epsilon}_{n-2 / 3}$, similar to the $\tilde{\epsilon}_{n}$ mode, but which has fractional moding on the $q= \pm 1$ sector:

$$
\begin{aligned}
\tilde{\epsilon}_{n-2 / 3}\left|\chi_{+1}\right\rangle & =\epsilon_{n-2 / 3}^{\dagger}\left|\chi_{+1}\right\rangle, \\
\tilde{\epsilon}_{n-2 / 3}\left|\chi_{-1}\right\rangle & =\epsilon_{n-2 / 3}\left|\chi_{-1}\right\rangle .
\end{aligned}
$$

Note that, unlike the $\tilde{\epsilon}_{n}$ modes, the $\tilde{\epsilon}_{n-2 / 3}$ modes map $q= \pm 1$ states to $q=0$ states.

In eq. (2.43) we wrote down a basis of states in the parafermion theory for the first three levels. Adding in the coordinate boson field, and tensoring $D$ copies together, we find all the states in the fermionic sector for these levels:

$$
\begin{array}{cl}
|\alpha, p\rangle & \text { level } 0, \\
\tilde{\epsilon}_{-2 / 3}^{\mu}|\alpha, p\rangle & \text { level } 2 / 3, \\
\tilde{\epsilon}_{-1}^{\mu}|\alpha, p\rangle+\alpha_{-1}^{\mu}|\alpha, p\rangle & \text { level } 1,
\end{array}
$$


The states at level $\ell$ have conformal dimension $\frac{p^{2}}{2}+\frac{D}{12}+\ell$. Note that, using the identities (2.44)-(2.47), these states can be written in many other equivalent forms.

Each of the $D$-fold tensor product components of the integer level states in (4.23) are in the ground state sector. Thus, in the string function notation of Appendix D, these states will contribute to a term $\left(c_{2}^{2}\right)^{D-2}$ in the (light-cone) partition function. Indeed, such a term (for $D=6$ ) certainly appears in the $A$ block of the FSS partition function (D.11). The level-2/3 state in (4.23), on the other hand, has one component in the projection sector. It would therefore contribute to a term $\left(c_{2}^{2}\right)^{D-3}\left(c_{2}^{4}\right)$ in the partition function; however, no such term appears in (D.11). This is analogous to the GSO projection in the Neveu-Schwarz sector of the superstring, where whole levels of states in the Fock space are projected out. Of course, in the superstring this does not happen in the Ramond sector since all modes automatically have integral moding there. In the FSS, due to the non-local nature of the operator algebra (the cuts in the $\epsilon^{(a)}$ OPEs), integer as well as $\pm 1 / 3$-moded operators appear in both the bosonic and fermionic sectors. It is thus natural to expect that a GSO-like projection removing whole levels of states will occur in both sectors.

Since the partition function found in Appendix D has contributions from states only at integral levels, we will concentrate solely on such states below. Though there is nothing preventing us from applying the physical state conditions to, say, the level$2 / 3$ states, presumably such states will decouple from all scattering amplitudes. We do not address the issue of scattering amplitudes in this paper.

A basis of states at level 2 is:

$$
\begin{gathered}
\alpha_{-2}^{\mu}|\alpha, p\rangle, \alpha_{-1}^{\mu} \alpha_{-1}^{\nu}|\alpha, p\rangle, \tilde{\epsilon}_{-1}^{\mu} \tilde{\epsilon}_{-1}^{\nu}|\alpha, p\rangle, \\
\alpha_{-1}^{\mu} \tilde{\epsilon}_{-1}^{\nu}|\alpha, p\rangle, \tilde{\epsilon}_{-2}^{\mu}|\alpha, p\rangle \\
\tilde{\epsilon}_{-2}^{(2) \mu}|\alpha, p\rangle
\end{gathered}
$$

for the states in the ground state sector, and

$$
\left.\begin{array}{l}
\tilde{\epsilon}_{-2 / 3}^{\mu} \tilde{\epsilon}_{-2 / 3}^{\nu} \tilde{\epsilon}_{-2 / 3}^{\lambda}|\alpha, p\rangle \\
\tilde{\epsilon}_{-2 / 3}^{\mu} \tilde{\epsilon}_{-2 / 3}^{\nu} \tilde{\epsilon}_{-2 / 3}^{(2) \lambda}|\alpha, p\rangle \\
\tilde{\epsilon}_{-2 / 3}^{\mu} \tilde{\epsilon}_{-2 / 3}^{(2) \nu} \tilde{\epsilon}_{-2 / 3}^{(2) \lambda}|\alpha, p\rangle \\
\tilde{\epsilon}_{-2 / 3}^{(2) \mu} \tilde{\epsilon}_{-2 / 3}^{(2) \nu} \tilde{\epsilon}_{-2 / 3}^{(2) \lambda}|\alpha, p\rangle
\end{array}\right\} \quad \text { for } \mu \neq \nu \neq \lambda
$$

for the projection sector states. Note that we have introduced the new notations, paralleling (4.6) and (4.22)

$$
\tilde{\epsilon}_{n}^{(2)} \equiv\left(\begin{array}{cc}
0 & \epsilon_{n}^{(+2)} \\
\epsilon_{n}^{(-2)} & 0
\end{array}\right), \quad n \in \mathbf{Z}
$$

and

$$
\begin{aligned}
& \tilde{\epsilon}_{n-2 / 3}^{(2)}\left|\chi_{+1}\right\rangle=\epsilon_{n-2 / 3}^{(+2)}\left|\chi_{+1}\right\rangle, \\
& \tilde{\epsilon}_{n-2 / 3}^{(2)}\left|\chi_{-1}\right\rangle=\epsilon_{n-2 / 3}^{(-2)}\left|\chi_{-1}\right\rangle
\end{aligned}
$$


following from the mode actions of $\epsilon^{( \pm 2)}$ on the $q= \pm 1$ sectors (see Fig. 1). Note that each of the states in (4.25) have three of their $D$-fold tensor product components in the projection sector. They will therefore contribute to a term of the form $\left(c_{2}^{2}\right)^{D-5}\left(c_{2}^{4}\right)^{3}$ in the partition function. Such a term does indeed appear in the $A$ block (D.11).

In a similar manner, a basis of states for any integer level can be built up from the $\alpha_{n}^{\mu}$ and $\epsilon_{r}^{(a)}$ modes acting on the ground state $|\alpha, p\rangle$. For example, using the mode algebra derived in Sect. 2, a basis of states in the one-component ground state sector can be written

$$
\tilde{\epsilon}_{-\ell(3 \ell+1)}^{(3 \ell+1)} \tilde{\epsilon}_{-m_{1}} \ldots \tilde{\epsilon}_{-m_{j}} \alpha_{-n_{1}} \ldots \alpha_{-n_{k}}|\alpha, p\rangle,
$$

where $\ell, m_{i}, n_{i} \in \mathbf{Z}, m_{i}, n_{i}>0$, and the sets $\left\{m_{1}, \ldots, m_{j}\right\}$ and $\left\{n_{1}, \ldots, n_{k}\right\}$ are "dictionary" ordered. The $\tilde{\epsilon}^{(a)}$ notation is the obvious generalization of the tilde notation we have used for other modes above. Note that in this notation $\tilde{\epsilon}^{(a)} \equiv \tilde{\epsilon}^{(-a)}$. The state in (4.28) has winding number $\pm(3 \ell+1 / 2)$ in the parafermion Fock space in other words, it is a descendent of the $\tilde{\epsilon}_{-\ell(3 \ell+1)}^{(3 \ell+1)}|\alpha\rangle \equiv \epsilon_{-\ell(3 \ell+1)-1 / 12}^{( \pm(3 \ell+1 / 2))}|0\rangle$ state. Indeed, an alternative basis of states, used in Sect. 2 to derive the parafermion characters (2.22), is for the one-component ground state sector

$$
\tilde{\epsilon}_{-\ell(3 \ell+1)}^{(3 \ell+1)} s_{-m_{1}} \ldots s_{-m_{j}} \alpha_{-n_{1}} \ldots \alpha_{-n_{k}}|\alpha, p\rangle .
$$

The recursion relation (2.37) and (2.38) can be used to express the (4.28) basis elements in terms of the $(4.29)$ basis elements.

A basis of states for the single component projection sector can similarly be written as

$$
\tilde{\epsilon}_{-3 \ell(\ell+1)-2 / 3}^{(3 \ell+1)} \tilde{\epsilon}_{-m_{1}} \ldots \tilde{\epsilon}_{-m_{j}} \alpha_{-n_{1}} \ldots \alpha_{-n_{k}}|\alpha, p\rangle .
$$

The general fermionic state is then a linear combination of tensor products of (4.28) or (4.30) states for each space-time dimension. Note that the (4.30) basis actually overcounts states by a factor of two. For example, $\tilde{\epsilon}_{-2 / 3}|\alpha\rangle$ and $\tilde{\epsilon}_{-2 / 3}^{(2)}|\alpha\rangle$ are actually the same state by the rule (2.34) derived in Sect. 2. Thus (4.25) has $2^{3}$ copies of each independent state. This overcounting could be rectified by restricting $\ell \geq 0$ in (4.30). However, allowing $\ell$ to run over the negative as well as positive integers (and thus allowing the doubling of states in the projection sector) will turn out to be necessary for the construction of a Lorentz-invariant GSO-like chiral projection in the fermionic sector.

\subsection{Chiral projection}

From experience with the superstring we expect that we will have to implement a GSO-like projection halving the number of degrees of freedom at all fermionic mass

\footnotetext{
${ }^{\dagger}$ A basis can also be written with just $\epsilon^{( \pm 1)}$ modes without using the higher winding number modes $\epsilon^{(a)}$ with $|a|>1$. However, in this case fractionally moded as well as integrally moded operators are required in the ground state sector.
} 
levels in order to have space-time supersymmetry. We will be able to do this if we can define an analog of the $(-1)^{F}$ operator in superstring theory, which we will call $(-1)^{\epsilon}$, that will enable us to generalize the Weyl condition on the massless states to all massive levels. A straightforward generalization from the $(K=2)$ superstring case leads one to guess the form

$$
(-1)^{\epsilon} \stackrel{?}{=} \gamma_{D+1}(-1)^{N(\epsilon)}
$$

where $N(\epsilon)$ is the number operator for the $\tilde{\epsilon}$ modes. However, this prescription does not tell us what signs to assign the winding modes. We will deduce below, from general arguments, the form of the $(-1)^{\epsilon}$ operator which is correct for all levels. It will turn out that the existence of such an operator consistent with Lorentz invariance follows from a counting argument relying on the Euler pentagonal number theorem.

Consider the general state in the ground state sector of the fermionic Fock spacethe $D$-fold tensor product of the basis states given in eq. (4.28):

$$
\left|\left\{\ell_{\lambda}\right\},\{m, \mu\},\{n, \nu\}\right\rangle_{g}=\left(\prod_{\lambda=0}^{D-1} \tilde{\epsilon}_{-\ell_{\lambda}\left(3 \ell_{\lambda}+1\right)}^{\left(3 \ell_{\lambda}+1\right), \lambda}\right) \tilde{\epsilon}_{-m_{1}}^{\mu_{1}} \ldots \tilde{\epsilon}_{-m_{j}}^{\mu_{j}} \alpha_{-n_{1}}^{\nu_{1}} \ldots \alpha_{-n_{k}}^{\nu_{k}}|\alpha, p\rangle
$$

(We will consider states with components in the projection sector later.) We can determine the prescription for counting the chirality of the winding modes $\tilde{\epsilon}^{(3 \ell+1), \lambda}$ from the requirement that there be an equal number of states with $(-1)^{\epsilon}=+1$ eigenvalue built on positive and negative chirality $\left(\gamma_{D+1}\right.$ eigenvalue $)$ states, $|\alpha, p\rangle_{ \pm}$, at each massive level of the Fock space. This is a necessary condition for the states at these levels to provide massive representations of the Lorentz group. For example, at the second mass level excluding the one winding mode state, there are $2 D^{2}+D$ states satisfying $(-1)^{\epsilon}=+1$ built on the positive chirality ground state (the states on the first line in eq. (4.24)), but only $D^{2}+D$ states built on the negative chirality ground state (the second line). Thus we must assign negative chirality to the winding mode operator $\tilde{\epsilon}^{(2) \mu}$ in the $D$ states on the third line of (4.24) in order to have left-right pairing. We can generalize this counting argument to arbitrary level and winding mode as follows.

Recall that the ground state sector of the fermionic Fock space is made up of a $D$-fold tensor product of the free coordinate boson theory with partition function $\eta^{-1}$, and the $\left[\phi_{1}^{1}\right]$ parafermion fields with character $\mathcal{Z}_{1}^{1}$ given in (2.22). Thus the character of the ground state sector is

$$
\operatorname{ch}_{g}(q)=2^{D / 2-1}\left(\frac{\mathcal{Z}_{1}^{1}}{\eta}\right)^{D}=2^{D / 2-1}\left(\frac{q^{1 / 12}}{\eta^{2}} \sum_{\ell} q^{3 \ell^{2}+\ell}\right)^{D} .
$$

where the factor $2^{D / 2-1}$ takes into account the dimension of the ground state, as well as the GSO-like projection. Note that this corresponds to only the term $2^{D / 2-1}\left(c_{2}^{2}\right)^{D-2}$ in the $A$ block partition function (D.11) of the fermionic sector, since we are not 
considering the projected sector states yet. The terms in the expression (4.33) for $\mathrm{ch}_{g}$ could have been read off directly from the basis of states (4.32). The two factors of $\eta^{-1}$ per dimension come from the boson modes $\alpha_{-n}^{\nu}$ and the parafermion energy operator modes $\tilde{\epsilon}_{-m}^{\mu}$. The summation is the contribution of the winding modes $\tilde{\epsilon}^{(3 \ell+1), \lambda}$.

Define now the chiral index

$$
I \equiv 2^{1-D / 2} \operatorname{Tr} \gamma_{D+1} q^{L_{0}}
$$

which gives at each level the difference in the number of even and odd chirality states (with the ground state multiplicity normalized to one). If this difference is zero for massive levels (as it must be in order that the states furnish good representations of the Lorentz group at all levels), then the massless ground state will be the only state to contribute to the chiral index. Thus we must choose the chiralities of the winding modes so that the chiral index $I=1$.

The ground state sector character $\mathrm{ch}_{g}$ can be rewritten as

$$
\operatorname{ch}_{g}(q)=2^{D / 2-1} \prod_{n=1}^{\infty}\left(1-q^{n}\right)^{-D} \prod_{m=1}^{\infty}\left(1-q^{m}\right)^{-D}\left(\sum_{\ell} q^{3 \ell^{2}+\ell}\right)^{D},
$$

where we have substituted the definition of the Dedekind $\eta$-function $\eta(q)=$ $q^{1 / 24} \prod_{m=1}^{\infty}\left(1-q^{m}\right)$. Since all the states contributing to this character (by hypothesis) satisfy the GSO-like condition $(-1)^{\epsilon}=+1$, the chirality of a given state will follow from the product of the chiralities of the parafermion fields. In particular, since each energy operator mode $\tilde{\epsilon}^{\mu}$ was found earlier to have chirality -1 , we should flip the sign of the $q^{m}$ terms in the $\eta^{-1}(q)$ factor corresponding to the $\tilde{\epsilon}$ modes. This reflects the fact that in the $(-1)^{\epsilon}=+1$ sector, states built on the ground state $|\alpha, p\rangle$ with an even number of $\tilde{\epsilon}$ excitations have even chirality, and states with an odd number of $\tilde{\epsilon}$ excitations have odd chirality. Thus, the chiral index $I$ is given by

$$
\begin{aligned}
I & =\prod_{n=1}^{\infty}\left(1-q^{n}\right)^{-D} \prod_{m=1}^{\infty}\left(1+q^{m}\right)^{-D}\left(\sum_{\ell} f(\ell) q^{3 \ell^{2}+\ell}\right)^{D} \\
& =\prod_{m=1}^{\infty}\left(1-q^{2 m}\right)^{-D}\left(\sum_{\ell} f(\ell) q^{3 \ell^{2}+\ell}\right)^{D}
\end{aligned}
$$

where $f(\ell)$ is the (unknown) chirality of the $\tilde{\epsilon}^{(3 \ell+1)}$ winding modes.

Recalling the Euler pentagonal number theorem [19]

$$
\prod_{n=1}^{\infty}\left(1-q^{n}\right)=\sum_{\ell}(-1)^{\ell} q^{\left(3 \ell^{2}+\ell\right) / 2}
$$

we see that if we make the choice

$$
f(\ell)=(-1)^{\ell}
$$


then the chiral index is

$$
I=\prod_{m=1}^{\infty}\left(1-q^{2 m}\right)^{-D} \prod_{n=1}^{\infty}\left(1-q^{2 n}\right)^{D}=1 .
$$

Thus the correct $(-1)^{\epsilon}$ operator for the ground state fermionic sector is given by

$$
(-1)^{\epsilon}=\gamma_{N+1}(-1)^{N(\epsilon)}(-1)^{N(\ell)},
$$

where we formally define the winding mode number operator by

$$
N(\ell)\left|\left\{\ell_{\lambda}\right\},\{m, \mu\},\{n, \nu\}\right\rangle_{g} \equiv\left(\sum_{\lambda=0}^{D-1} \ell_{\lambda}\right)\left|\left\{\ell_{\lambda}\right\},\{m, \mu\},\{n, \nu\}\right\rangle_{g}
$$

on the basis of states of the ground state sector given in (4.32).

We now turn to the states in the projection sector. As noted earlier, all these states are massive. Therefore, by the argument outlined above, the chiral index must be zero in the projection sector to be consistent with Lorentz invariance. In other words, at each mass level there should be an equal number of left- and right-chirality states. Recall that the full projection sector is the tensor product of $D-3$ onecomponent ground state sectors and 3 one-component projection sectors. Using the one-component bases of states, (4.28) and (4.30), we can easily write down a basis of states for the full projection sector:

$$
\begin{aligned}
& |\{\ell, \lambda\},\{m, \mu\},\{n, \nu\}\rangle_{p}= \\
& \left(\prod_{i=1}^{D-3} \tilde{\epsilon}_{-\ell_{i}\left(3 \ell_{i}+1\right)}^{\left(3 \ell_{i}+1\right), \lambda_{i}}\right)\left(\prod_{i=D-2}^{D} \tilde{\epsilon}_{-3 \ell_{i}\left(\ell_{i}+1\right)-2 / 3}^{\left(3 \ell_{i}+1\right), \lambda_{i}}\right) \tilde{\epsilon}_{-m_{1}}^{\mu_{1}} \ldots \tilde{\epsilon}_{-m_{j}}^{\mu_{j}} \alpha_{-n_{1}}^{\nu_{1}} \ldots \alpha_{-n_{k}}^{\nu_{k}}|\alpha, p\rangle,
\end{aligned}
$$

where where together the $D \lambda_{i}$ s span the set $\{0,1, \ldots, D-1\}$ of space-time indices. The partition function for the projection sector is given by a similar argument as above:

$$
\begin{aligned}
\operatorname{ch}_{p}(q) & =2^{D / 2-1}\left(\frac{\mathcal{Z}_{1}^{1}}{\eta}\right)^{D-3}\left(\frac{\mathcal{Z}_{1}^{0}+\mathcal{Z}_{-1}^{0}}{\eta}\right)^{3} \\
& =2^{D / 2-1}\left(\frac{q^{1 / 12}}{\eta^{2}} \sum_{\ell} q^{3 \ell^{2}+\ell}\right)^{D-3}\left(\frac{2 q^{3 / 4}}{\eta^{2}} \sum_{p} q^{3 p(p+1)}\right)^{3} .
\end{aligned}
$$

This corresponds to the term $2^{D / 2-1}\left(c_{2}^{2}\right)^{D-5}\left(2 c_{2}^{4}\right)^{3}$ in the $A$ block partition function (D.11). The first summation comes from the winding modes in the $D-3$ onecomponent ground state sectors, while the second summation comes from the winding modes in the 3 one-component projection sectors. Computing the chiral index using the definition of $(-1)^{\epsilon}$ derived above, we find

$$
I=8 q^{2} \prod_{m=1}^{\infty}\left(1-q^{2 m}\right)^{-3}\left(\sum_{\ell=-\infty}^{+\infty} f(\ell) q^{3 \ell(\ell+1)}\right)^{3}
$$


where we have used the fact, derived above, that $I=1$ for each of the $D-3$ ground state sector components separately. Thus the summation in (4.44) is over the winding numbers of the operators $\tilde{\epsilon}^{(3 \ell+1)}$ contributing to the three projection sector components. If we take the same prescription for the chirality of these winding modes as we took in the ground state sector, namely $f(\ell)=(-1)^{\ell}$, it is easy to see that the chiral index (4.44) vanishes due to the symmetry $\ell \rightarrow-\ell-1$ of the sum, which is the desired result.

To summarize, the correct form of the $(-1)^{\epsilon}$ projection operator consistent with Lorentz invariance is given by eq. (4.40) for the fermionic sector of the FSS, where the winding mode number operator $N(\ell)$ is defined by (4.41) on ground state sector states, and by

$$
N(\ell)|\{\ell, \lambda\},\{m, \mu\},\{n, \nu\}\rangle_{p} \equiv\left(\sum_{i=1}^{D} \ell_{i}\right)|\{\ell, \lambda\},\{m, \mu\},\{n, \nu\}\rangle_{p}
$$

on projection sector states. We emphasize that the existence of this GSO-like projection compatible with Lorentz invariance was by no means guaranteed. Indeed, we have seen that it works only through the use of the non-trivial identity (4.37).

\section{The bosonic sector}

In this section we construct the full space of states in the bosonic sector of a $D$-dimensional open $K=4 \mathrm{FSS}$. We then solve for the subset of states at low-lying mass levels that satisfy the physical state conditions. The ground state is found to be tachyonic, while the first excited state is a massless vector particle. The partition function for the closed FSS derived in Appendix D implies that the tachyonic state is removed by an analog of the GSO projection.

\subsection{Bosonic Fock space}

We build the bosonic sector of the FSS Fock space by the action of the coordinate boson modes $\alpha_{n}^{\mu}$ and the parafermion energy operator modes $\epsilon_{r}^{( \pm 1)}$ on the ground state

$$
|p\rangle \equiv: \mathrm{e}^{i p \cdot X(0)}:|0\rangle \text {. }
$$

In Sect. 2 we showed that in the single space-time component theory, the action of the energy operator modes on the identity operator (vacuum state) $|0\rangle$ correspond to the parafermion sectors $\left[\phi_{0}^{0}\right],\left[\phi_{0}^{1}\right]$ and $\left[\phi_{0}^{2}\right]$. Recall that $\left[\phi_{0}^{0}\right]$ and $\left[\phi_{0}^{2}\right]$ consist of the set of fields $\epsilon^{(3 n)}$ and $\partial \rho$ with integer conformal dimensions and $\mathbf{Z}_{3}$ charge zero, whereas $\left[\phi_{0}^{1}\right]$ consists of the $\epsilon^{(3 n \pm 1)}$ fields of dimension $\mathbf{Z}+1 / 3$ and $\mathbf{Z}_{3}$ charge \pm 1 . The allowed modings and actions of the integral winding-number fields $\epsilon^{(a)}, a \in \mathbf{Z}$, on different $\mathbf{Z}_{3}$ sector fields in the bosonic sector is also summarized by Fig. 1. 
In eq. (2.39) we wrote down a basis of states in the parafermion theory for the first four levels. Adding in the coordinate boson field, and tensoring $D$ copies together, we find the complete list of states in the bosonic sector for these levels:

$$
\begin{array}{rc}
|p\rangle & \text { level } 0 \\
\epsilon_{-1 / 3}^{( \pm 1) \mu}|p\rangle & 1 / 3 \\
\epsilon_{-1 / 3}^{\mu_{1}} \epsilon_{-1 / 3}^{\mu_{2}}|p\rangle & 2 / 3, \\
\alpha_{-1}^{\mu}|p\rangle+\epsilon_{-2 / 3}^{( \pm 1) \mu} \epsilon_{-1 / 3}^{(\mp 1) \mu}|p\rangle+\epsilon_{-1 / 3}^{\mu_{1}} \epsilon_{-1 / 3}^{\mu_{2}} \epsilon_{-1 / 3}^{\mu_{3}}|p\rangle & 1 \\
\epsilon_{-1 / 3}^{( \pm 1) \mu} \alpha_{-1}^{\nu}|p\rangle+\epsilon_{-1}^{( \pm 1) \mu} \epsilon_{-1 / 3}^{( \pm 1) \mu}|p\rangle+\epsilon_{-4 / 3}^{( \pm 1) \mu}|p\rangle+\epsilon_{-1 / 3}^{\mu_{1}} \epsilon_{-1 / 3}^{\mu_{2}} \epsilon_{-1 / 3}^{\mu_{3}} \epsilon_{-1 / 3}^{\mu_{4}}|p\rangle & 4 / 3
\end{array}
$$

The mode operators in the $\epsilon_{-1 / 3}^{\mu_{1}} \cdots \epsilon_{-1 / 3}^{\mu_{n}}|p\rangle$ states in (5.2) are to be understood to be either $\epsilon^{(+1) \mu_{i}}$ or $\epsilon^{(-1) \mu_{i}}$ modes, independently for each factor, and that in these terms $\mu_{i} \neq \mu_{j}$. Thus, for example, $\epsilon_{-1 / 3}^{\mu_{1}} \epsilon_{-1 / 3}^{\mu_{2}} \epsilon_{-1 / 3}^{\mu_{3}}|p\rangle$ represents $8\left(\begin{array}{c}D \\ 3\end{array}\right)$ independent states for fixed $p^{\mu}$. The states at level $\ell$ have conformal dimension $\frac{p^{2}}{2}+\ell$. Note that we have used the identities $(2.40)-(2.42)$ to write these states in terms of $\epsilon^{( \pm 1)}$ modes only. Using the machinery developed in Sect. 2, this can be done for the whole bosonic Fock space.

We can now make a correspondence between these states and the light-cone spectrum of the $D=6$ FSS found in Appendix D. Recall that the ground state, corresponding to a term $\left(c_{0}^{0}\right)^{D-2}$ in the partition function does not appear in the $A$ block of the FSS partition function (D.11). This is a reflection of a GSO-like projection in the FSS spectrum. In general, only the level $\mathbf{Z}+\frac{1}{3}$ states survive this projection. Thus, the $2 D$ states at level $1 / 3$ in $(5.2)$ contribute to the $(D-2)\left(c_{0}^{0}\right)^{D-3}\left(c_{0}^{2}\right)$ term in the partition function. We will see below how the physical state conditions reduce the multiplicity of this state from $2 D$ to $D-2$. At level $4 / 3$, the first three states in (5.2) contribute to the same term in the partition function as the level $1 / 3$ state; the last state, however, contributes to the $\left(c_{0}^{2}\right)^{4}$ term in (D.11). This is one of the "internal projection" states in the bosonic sector.

\subsection{Physical state conditions and the massless vector particle}

Now we wish to impose the physical state conditions

$$
\begin{aligned}
\left(L_{0}-v\right)|\phi\rangle & =0, \\
\left(G_{0}-\beta\right)|\phi\rangle & =0, \\
L_{n}|\phi\rangle=G_{n / 3}|\phi\rangle & =0, \quad n>0 .
\end{aligned}
$$

We will determine the intercepts $v$ and $\beta$ by demanding that extra sets of null states appear at their critical values. Note that, unlike in the usual superstring case, both integer and fractional moding of the currents are allowed in the bosonic sector. 
Let us start with the general level zero state

$$
\left|\phi_{0}\right\rangle=\zeta(p)|p\rangle
$$

where $\zeta(p)$ is an arbitrary (scalar) wave function. Clearly only the $L_{0}$ and $G_{0}$ conditions are potentially non-vanishing on $\left|\phi_{0}\right\rangle$. From the moding diagram, Fig. 1, it is also easy to see that $G_{0}\left|\phi_{0}\right\rangle=0$. Finally, since $L_{0}\left|\phi_{0}\right\rangle=\frac{p^{2}}{2}\left|\phi_{0}\right\rangle$, the physical state conditions on $\left|\phi_{0}\right\rangle$ are $v=p^{2} / 2$ and $\beta=0$. We will show below that $\beta \neq 0$ at its critical value, and thus that the tachyonic state must decouple. Note that this is different from what happens in the usual superstring, where the physical state conditions by themselves do not remove the tachyonic states. (The GSO projection is only required at the string loop level.) This new situation is clearly related to the fact that in the FSS the bosonic sector admits integral moding of the fractional supercurrent.

We now consider the general level 1/3 state:

$$
\left|\phi_{1 / 3}\right\rangle=\left(\zeta_{\nu} \epsilon_{-1 / 3}^{\nu}+\zeta_{\nu}^{\dagger} \epsilon_{-1 / 3}^{\nu^{\nu}}\right)|p\rangle
$$

where $\zeta_{\mu}$ and $\zeta_{\mu}^{\dagger}$ are polarization vectors, and we have introduced the notation

$$
\epsilon=\epsilon^{(+1)}, \text { and } \epsilon^{\dagger}=\epsilon^{(-1)} \text {. }
$$

Acting on this state, the non-trivial physical state conditions are $L_{0}, G_{0}$ and $G_{1 / 3}$. The $L_{0}$ condition is easily seen to give

$$
\left(L_{0}-v\right)\left|\phi_{1 / 3}\right\rangle=0 \quad \Longrightarrow \quad v=\frac{p^{2}}{2}+\frac{1}{3} .
$$

Using the mode expansion of the $G$ current (3.16) we can write the action of the $G_{1 / 3}$ mode on $\left|\phi_{1 / 3}\right\rangle$ in terms of component fields as

$$
G_{1 / 3}\left|\phi_{1 / 3}\right\rangle=\frac{1}{\sqrt{2}} \sum_{n} \alpha_{n, \mu}\left[\epsilon_{1 / 3-n}^{\mu}+\epsilon_{1 / 3-n}^{\dagger \mu}\right]\left(\zeta_{\nu} \epsilon_{-1 / 3}^{\nu}+\zeta_{\nu}^{\dagger} \epsilon_{-1 / 3}^{\dagger^{\nu}}\right)|p\rangle
$$

since on this state there is no contribution from the $\epsilon^{( \pm 2)}$ terms in the current. Now, the expression

$$
\epsilon_{1 / 3}^{\dagger \mu} \epsilon_{-1 / 3}^{\nu}|p\rangle
$$

is only well-defined, with respect to the moding rules given in Fig. 1, for $\mu=\nu$. For $\mu \neq \nu$, the moding is inappropriate, since $\epsilon_{1 / 3}^{\dagger \mu}$ is acting on the vacuum state with $\mathbf{Z}_{3}$-charge 0 for the $\mu$-th component, and thus has the wrong moding. However, recall that in Sect. 3 we learned how to deal with this problem - the lesson being that "wrong" moding operators should be formally set to zero. With this understanding, we can give a covariant meaning to the expression (5.9):

$$
\epsilon_{1 / 3}^{\dagger^{\mu}} \epsilon_{-1 / 3}^{\nu}|p\rangle=g^{\mu \nu}|p\rangle,
$$


where $g^{\mu \nu}$ is the Minkowski metric. Here we have used the one-component commutation relations for the $\mu=\nu$ case. Thus

$$
\begin{aligned}
G_{1 / 3}\left|\phi_{1 / 3}\right\rangle & =\frac{1}{\sqrt{2}} \alpha_{0} \cdot\left(\epsilon_{1 / 3}+\epsilon_{1 / 3}^{\dagger}\right)\left(\zeta \cdot \epsilon_{-1 / 3}+\zeta^{\dagger} \cdot \epsilon_{-1 / 3}^{\dagger}\right)|p\rangle \\
& =\frac{1}{\sqrt{2}} p \cdot\left(\zeta+\zeta^{\dagger}\right)|p\rangle,
\end{aligned}
$$

where all other terms vanish by either using the single-component GCRs, eq. (2.27) for the cases where $\mu=\nu$, or the annihilation property of "wrong-moding" operators for $\mu \neq \nu$. The $G_{1 / 3}$ physical state condition then gives

$$
G_{1 / 3}\left|\phi_{1 / 3}\right\rangle=0 \Longrightarrow p \cdot\left(\zeta+\zeta^{\dagger}\right)=0 \text {. }
$$

Finally, let us compute the $G_{0}$ condition on $\left|\phi_{1 / 3}\right\rangle$. From (3.16) we have

$$
G_{0}\left|\phi_{1 / 3}\right\rangle=\sum_{\mu}\left\{\frac{1}{\sqrt{2}} \sum_{n} \alpha_{n, \mu}\left(\epsilon_{n}^{\mu}+\epsilon_{n}^{\dagger \mu}\right)+\frac{1}{2}\left(\epsilon_{0}^{(+2) \mu}+\epsilon_{0}^{(-2) \mu}\right)\right\}\left|\phi_{1 / 3}\right\rangle .
$$

The first term vanishes since $\epsilon_{n}^{\mu}$ for $n \in \mathbf{Z}$ has the "wrong" moding for all the components of $\left|\phi_{1 / 3}\right\rangle$ (see Fig. 1). However, the second term, involving the $\epsilon_{0}^{( \pm 2) \mu}$ modes, does not vanish. Recall that we can write the $\epsilon^{( \pm 2)}$ modes in terms of the $\epsilon$ and $\epsilon^{\dagger}$ modes (3.17), so that

$$
\sum_{\mu} \epsilon_{0}^{( \pm 2) \mu}=g_{\mu \nu} \sum_{\ell=0}^{\infty} c_{\ell}^{(-5 / 3)}\left[\epsilon_{-\frac{1}{3}-\ell}^{( \pm 1) \mu} \epsilon_{\frac{1}{3}+\ell}^{( \pm 1) \nu}+\epsilon_{-\frac{4}{3}-\ell}^{( \pm 1) \mu} \epsilon_{\frac{4}{3}+\ell}^{( \pm 1) \nu}\right]
$$

when acting on a state with $\mathbf{Z}_{3}$ charge $q= \pm 1$. (By Fig. $1, \epsilon_{0}^{( \pm 2)}$ does not act on states with $q=0$.) Now, by simple dimensional considerations, all $\epsilon_{n}^{(a)}$ modes annihilate $\left|\phi_{1 / 3}\right\rangle$ if $n>1 / 3$. This drastically reduces the sum in (5.14), so that we have

$$
\begin{aligned}
G_{0}\left|\phi_{1 / 3}\right\rangle & =\frac{1}{2}\left(\epsilon_{-1 / 3} \cdot \epsilon_{1 / 3}+\epsilon_{-1 / 3}^{\dagger} \cdot \epsilon_{1 / 3}^{\dagger}\right)\left(\zeta \cdot \epsilon_{-1 / 3}+\zeta^{\dagger} \cdot \epsilon_{-1 / 3}^{\dagger}\right)|p\rangle \\
& =\frac{1}{2}\left(\zeta^{\dagger} \cdot \epsilon_{-1 / 3}+\zeta \cdot \epsilon_{-1 / 3}^{\dagger}\right)|p\rangle
\end{aligned}
$$

where we have used the $\mathbf{Z}_{4}$ parafermion identities

$$
\epsilon_{1 / 3}^{( \pm 1)} \epsilon_{-1 / 3}^{(\mp 1)}|0\rangle=|0\rangle
$$

which follow from (2.34). Therefore the $G_{0}$ physical state condition implies

$$
\left(G_{0}-\beta\right)\left|\phi_{1 / 3}\right\rangle=0 \quad \Longrightarrow \quad \zeta_{\mu}=\zeta_{\mu}^{\dagger} \text { and } \beta=\frac{1}{2}
$$

Thus the physical state conditions (5.7), (5.12) and (5.17) reduce the original $2 D$ components of the $\zeta_{\mu}$ and $\zeta_{\mu}^{\dagger}$ polarization vectors to only $D-1$ independent 
components. In addition, there is a critical value of the intercept $v$ for which an additional degree of freedom is removed. In particular, when

$$
v=\frac{1}{3}
$$

by the $L_{0}$ condition (5.7), we find that $p^{2}=0$, so that $\left|\phi_{1 / 3}\right\rangle$ describes a massless vector particle. Thus, the state with polarization $\zeta_{\mu} \propto p_{\mu}$ has zero norm, and there are only $D-2$ physical polarizations.

\section{$6 \quad$ Higher mass levels}

So far in this paper we have constructed the Fock space of states for the (open) $K=4$ fractional superstring, and have shown that the simplest guess for the physical state conditions provides the correct equations of motion for the lowest-lying states in the fractional superstring spectrum. Also, demanding the presence of extra null states (or, equivalently, demanding massless vector and spinor particles with the same number of propagating degrees of freedom as in the partition function), fixed the intercepts in the space-time bosonic and fermionic sectors. This is all in agreement with the $K=4$ FSS partition function, whose derivation is reviewed in Appendix D.

The next step is, clearly, to examine higher mass levels in the FSS Fock space. However, at these levels we run into two separate problems, which may or may not be related: Lorentz non-invariance and the failure of the physical state conditions to implement the internal projection. We will describe below in some detail how these problems arise, and then we will briefly outline a few possible ways in which they may be resolved. We would like to point out at the outset that we do not have a clear resolution of these problems.

\subsection{Lorentz non-invariance}

We constructed the FSS Fock space by tensoring together $D$ copies of the $\mathbf{Z}_{4}$ parafermion conformal field theory, with the aim of describing the FSS spectrum in $D$ flat spacetime dimensions. However, a priori, taking the tensor product only ensures a permutation symmetry among the dimensions, and not the full rotational Lorentz symmetry.

A concrete realization of this point is encountered at the first massive level in the fermionic sector. In particular, we will impose the physical state conditions (3.23) and (3.27) on the level 1 states, and show that the resulting equations of motion are not Lorentz covariant.

Consider the general state at level 1:

$$
\left|\psi_{1}\right\rangle=\alpha_{-1}^{\mu}|\alpha, p\rangle u_{\mu}^{\alpha}(p)+\tilde{\epsilon}_{-1}^{\mu}|\alpha, p\rangle w_{\mu}^{\alpha}(p)
$$

where $u_{\mu}^{\alpha}$ and $w_{\mu}^{\alpha}$ are spinor wave functions. The $L_{n}$ and $G_{r}$ modes for $n, r>1$ are easily seen to identically annihilate $\left|\psi_{1}\right\rangle$. In addition, by eq. (3.18), the $G_{1}$ condition 
is not independent of the $L_{1}$ and $G_{0}$ conditions, and the $G_{2 / 3}$ condition identically annihilates $\left|\psi_{1}\right\rangle$. Thus, the only physical state conditions that need to be checked are those corresponding to $L_{0}, L_{1}, G_{0}$ and $G_{1 / 3}$. The $L_{0}$ and $L_{1}$ conditions are Lorentz covariant. In particular, since $\left|\psi_{1}\right\rangle$ has conformal dimension $p^{2} / 2+D / 12+1$, the $L_{0}$ condition, with the value of the intercept derived in Sect. 4 , gives

$$
\left(L_{0}-D / 12\right)\left|\psi_{1}\right\rangle=0 \Longrightarrow p^{2}=-2 .
$$

Thus the level 1 physical state corresponds to particles of mass $m=\sqrt{2}$. Using the commutators (2.56), one also finds that

$$
L_{1}|\psi\rangle=0 \Longrightarrow p \cdot u(p)+\frac{1}{3} \not(p)=0,
$$

where we have replaced $\tilde{\epsilon}_{0}^{\mu}$ with $\gamma^{\mu}$ when acting on the ground state $|\alpha, p\rangle$.

To evaluate the other physical state conditions, we must use the generalized commutation relations (GCRs) of the $\tilde{\epsilon}^{\mu}$ fields. The GCR of $\tilde{\epsilon}^{\mu}$ and $\tilde{\epsilon}^{\nu}$ is given by the single-component relation (2.27) when $\mu=\nu$. When $\mu \neq \nu$, however, all modes simply anticommute. In particular, when acting on the ground state $\tilde{\epsilon}_{0}^{\mu}$ and $\tilde{\epsilon}_{-1}^{\nu}$ satisfy

$$
\begin{aligned}
\left(\tilde{\epsilon}_{0}^{\mu} \tilde{\epsilon}_{-1}^{\nu}+\tilde{\epsilon}_{-1}^{\nu} \tilde{\epsilon}_{0}^{\mu}\right)|\alpha, p\rangle & =0, \quad \mu \neq \nu, \\
\left(\tilde{\epsilon}_{0}^{\mu} \tilde{\epsilon}_{-1}^{\nu}+\frac{1}{3} \tilde{\epsilon}_{-1}^{\nu} \tilde{\epsilon}_{0}^{\mu}\right)|\alpha, p\rangle & =0, \quad \mu=\nu .
\end{aligned}
$$

The $G_{0}$ physical state condition can now be evaluated:

$$
\begin{aligned}
G_{0}\left|\psi_{1}\right\rangle= & \frac{1}{\sqrt{2}}\left[\alpha_{1} \cdot \tilde{\epsilon}_{1}+\alpha_{0} \cdot \tilde{\epsilon}_{0}+\tilde{\epsilon}_{-1} \cdot \alpha_{1}\right]\left|\psi_{1}\right\rangle \\
= & \frac{1}{\sqrt{2}}\left[\alpha_{-1} \cdot\left(\not p u+\frac{2}{3} w\right)+\tilde{\epsilon}_{-1} \cdot(u-\not p w)\right. \\
& \left.+\frac{2}{3} \sum_{\mu} \tilde{\epsilon}_{-1}^{\mu} p^{\mu} \gamma^{\mu} w^{\mu}\right]|\alpha, p\rangle,
\end{aligned}
$$

or, $G_{0}\left|\psi_{1}\right\rangle=0$ implies that

$$
\begin{aligned}
\not p u_{\mu}+\frac{2}{3} w_{\mu} & =0, \\
u_{\mu}-\frac{1}{3} \not p w_{\mu}-\frac{2}{3}\left(\sum_{\nu \neq \mu} p^{\nu} \gamma^{\nu}\right) w_{\mu} & =0 .
\end{aligned}
$$

Compatibility of these equations with the $L_{0}$ condition implies the Lorentz noncovariant constraint

$$
\left(\sum_{\nu \neq \mu} p^{\nu} \gamma^{\nu}\right) w_{\mu}=0
$$


Note that even though the $G_{1}$ physical state condition is not independent of the $G_{0}$ condition, since $\left[L_{1}, G_{0}\right]=\frac{1}{3} G_{1}$, it does, however, give rise to a Lorentz covariant constraint:

$$
G_{1}\left|\psi_{1}\right\rangle=0 \quad \Longrightarrow \quad \not u+\frac{2}{3} p \cdot w=0 .
$$

Similarly, the $G_{1 / 3}$ condition can be evaluated using the fact that, on the ground state,

$$
\begin{aligned}
\tilde{\epsilon}_{1 / 3}^{\mu} \tilde{\epsilon}_{-1}^{\nu}|\alpha, p\rangle & =0, \quad \mu \neq \nu, \\
\left(\tilde{\epsilon}_{1 / 3}^{\mu} \tilde{\epsilon}_{-1}^{\nu}+\frac{2}{3} \tilde{\epsilon}_{-2 / 3}^{\nu} \tilde{\epsilon}_{0}^{\mu}\right)|\alpha, p\rangle & =0, \quad \mu=\nu .
\end{aligned}
$$

Thus

$$
\begin{aligned}
G_{1 / 3}\left|\psi_{1}\right\rangle & =\left\{\frac{1}{\sqrt{2}}\left[\tilde{\epsilon}_{-2 / 3} \cdot \alpha_{1}+\alpha_{0} \cdot \tilde{\epsilon}_{1 / 3}\right]+\tilde{\epsilon}_{-2 / 3} \cdot \tilde{\epsilon}_{1}\right\}\left|\psi_{1}\right\rangle \\
& =\frac{1}{\sqrt{2}} \tilde{\epsilon}_{-2 / 3}^{\mu}\left[u_{\mu}+\sqrt{2} w_{\mu}-\frac{2}{3} p^{\mu} \gamma^{\mu} w^{\mu}\right]|\alpha, p\rangle
\end{aligned}
$$

where we have used (3.17) to rewrite the $\tilde{\epsilon}_{1 / 3}^{(2)}$ term as an expression quadratic in $\tilde{\epsilon}^{(1)}$ modes. It is clear from (6.11) that the $G_{1 / 3}$ condition gives rise to the same Lorentz non-invariant piece found in the $G_{0}$ condition. Using (6.8), the $G_{1 / 3}$ physical state condition becomes

$$
G_{1 / 3}\left|\psi_{1}\right\rangle=0 \Longrightarrow u_{\mu}+\sqrt{2} w_{\mu}-\frac{2}{3} \not p w_{\mu}=0 .
$$

The only solution to the physical state conditions is $w_{\mu}=u_{\mu}=0$. So, not only are the $L_{n}$ and $G_{r}$ physical state conditions Lorentz non-covariant at the first massive level, but they are also too strong; they allow no propagating states, even though the partition function predicts 32 such states at this level (see Appendix D).

\subsection{Internal projection}

A second problem that arises at the higher mass levels concerns the presence of extra cancellations between states of the FSS Fock space, which have no analog in usual superstring.

Recall from the discussion in Appendix D that we can identify the form of the vertex operators for space-time bosons (D.13) or fermions (D.14) in the $A$ block of the partition function on the basis of a statistics selection rule. By matching $\mathbf{Z}_{4}$ parafermion quantum numbers, we can easily identify the bosonic and fermionic pieces of the $A$ block partition function, which we write separately as

$$
\begin{aligned}
& A_{b}=4\left(c_{0}^{0}+c_{0}^{4}\right)^{3}\left(c_{0}^{2}\right)-4\left(c_{0}^{2}\right)^{4}, \\
& A_{f}=4\left(c_{2}^{2}\right)^{4}-32\left(c_{2}^{2}\right)\left(c_{2}^{4}\right)^{3},
\end{aligned}
$$


so that $A=A_{b}-A_{f}$. The puzzling feature of these identifications is that not all the terms contributing to space-time bosons have positive coefficients, and likewise not all space-time fermions have negative coefficients. By the supersymmetric vanishing of $A$ (D.12), as functions of the modular parameter $q$ we have $A_{b}=A_{f}$. It turns out [13] that the fermionic (or bosonic) piece satisfies the identity

$$
A_{f}=4\left(\prod_{n=1}^{\infty} \frac{1+q^{n}}{1-q^{n}}\right)^{4}
$$

which gives the same counting of physical degrees of freedom as the Ramond sector in 6 space-time dimensions. Since the coefficients in the $q$-expansion of the right-hand side are all positive, we are led to view the minus signs in $A_{b}$ and $A_{f}$ as "internal projections" (or cancellations) of degrees of freedom in the fractional superstring.

The problem with implementing the internal projections in the framework of this paper can be described as follows. Note that the internal projection occurs only at or above the first massive level in the bosonic sector and the second massive level in the fermionic sector. For example, consider the Fock space description of the states at the first massive level in the bosonic sector:

$$
\begin{aligned}
\left|\phi_{1}\right\rangle=\left\{A_{\mu} \tilde{\epsilon}_{-4 / 3}^{\mu}\right. & +B_{\mu \nu} \alpha_{-1}^{\mu} \tilde{\epsilon}_{-1 / 3}^{\nu}+C_{\mu \nu} s_{-1}^{\mu} \tilde{\epsilon}_{-1 / 3}^{\nu} \\
& \left.+D_{\mu \nu \rho \sigma} \tilde{\epsilon}_{-1 / 3}^{\mu} \tilde{\epsilon}_{-1 / 3}^{\nu} \tilde{\epsilon}_{-1 / 3}^{\rho} \tilde{\epsilon}_{-1 / 3}^{\sigma}\right\}
\end{aligned}
$$

where $\tilde{\epsilon}$ can stand for either $\epsilon^{(+1)}$ or $\epsilon^{(-1)}, \mu \neq \nu \neq \rho \neq \sigma$ in the last term, and $A, B, C, D$ are polarization tensors. The first three terms in (6.15) all correspond to contributions from terms with positive coefficients in $A_{b}(6.13)$, while the last term, since it involves four $\tilde{\epsilon}^{\prime}$ s, corresponds to the projection term $-4\left(c_{0}^{2}\right)^{4}$ in $A_{b}$. Thus, even though the last term adds more states to the FSS Fock space, once the physical state conditions are implemented they must actually subtract states. This implies that the physical state conditions must, at least, mix the states in the Fock space corresponding to the "ground state" and "projection" sectors.

However, the general form of the physical state conditions, assumed in Sect. 3, is, schematically, in terms of the $\alpha$ and $\epsilon$ modes,

$$
\begin{aligned}
& L_{n} \sim \sum_{m}\left(\alpha_{m} \cdot \alpha_{n-m}+\epsilon_{m} \cdot \epsilon_{n-m}\right) \\
& G_{r} \sim \sum_{m}\left(\alpha_{m} \cdot \epsilon_{r-m}+\epsilon_{m} \cdot \epsilon_{r-m}\right) .
\end{aligned}
$$

It is easy to see that operators of this form can never mix the first three terms with the last one in $\left|\phi_{1}\right\rangle$. This statement holds generally for all the higher mass levels in the FSS Fock space as well.

Note that the counting of degrees of freedom after the internal projection implied by (6.14) is precisely that of $D-2$ pairs of world-sheet bosons and fermions, similar 
to that of the usual superstring. Thus the asymptotic degeneracy of states in $A_{b}$ or $A_{f}$ corresponds to the effective central charge

$$
c_{\text {eff }}=(D-2) \cdot \frac{3}{2},
$$

or $c_{\text {eff }}=6$ for the $K=4$ FSS where $D=6$. Since each dimension in the FSS Fock space corresponds to a CFT with central charge $c_{0}=2$, we see that, with the internal projection, the physical state conditions must remove the equivalent of three dimensions' worth of states, and not just the timelike and longitudinal ones expected from a large critical string gauge invariance.

\subsection{Possible resolutions}

We will now describe possible modifications of the framework presented in this paper, which may solve the above-mentioned problems. First, we will discuss some of the assumptions which underlie the discussions of the last two subsections. The most important is Lorentz invariance. Clearly, if one were willing to give up Lorentz invariance, the occurence of non-invariant physical state conditions would not be a problem. Similarly, the existence of the internal projection was based on an identification of space-time bosons and fermions from their statistics selection rules. Without Lorentz invariance, this argument also has no force. So, it is a logical possibility that the above-mentioned problems are simply an indication that we have to give up Lorentz invariance above the massless level of states. (String interactions will presumably then give Lorentz non-invariant contributions to the effective action for the massless states as well.)

However, the fact that there does exist a Lorentz-covariant description of the massless state, that the A block of the partition function admits a separation into pieces satisfying statistics selection rules, and that the chiral counting argument described in Sect. 4.4 works, all hint that a Lorentz-covariant interpretation of the $K=4$ FSS should exist. Two possible ways in which such an interpretation could be realized are to either change the physical state conditions or to change the Fock space on which they act. We will describe below how these proposals can be systematically explored.

The idea behind changing the physical state conditions is to add terms to the $G_{r}$ that cancel the Lorentz non-covariant pieces in the equations of motion they generate. One could do this level by level in the FSS Fock space. For example, we saw above that the $G_{0}$ condition gives rise to Lorentz non-covariant terms in the equations of motion of the first massive fermion states. An example of the kind of modification that could cancel those terms is

$$
G_{0} \longrightarrow \tilde{G}_{0}=G_{0}+\kappa G_{-1 / 3} G_{1 / 3}+\ldots,
$$

where $\kappa$ is a parameter to be fixed by the requirement of Lorentz invariance. Note that the new mode operator $\tilde{G}_{0}$ has the same action on the massless states as did the 
old. The $\tilde{G}_{0}$ proposed in (6.18) is just meant to be illustrative of this idea; in fact there are many more terms that contribute at the first massive level and conceivably could contribute to (6.18), since there is no reason that $\tilde{G}_{0}$ must be manifestly covariant. All we require is that the Lorentz non-covariance of the tensor-product Fock space cancels against appropriate non-covariant physical state conditions.

The modified $\tilde{G}_{r}$ must satisfy other conditions besides the requirement that they yield covariant equations of motion. In particular, they must implement the internal projections described above, and must give rise to extra towers of null states, indicative of the critical string gauge invariance. There is at least a hope of implementing the internal projection, since the modified $\tilde{G}_{r}$ 's can now include terms that mix the projection and ground state sectors, and an extra condition (or perhaps $\tilde{G}_{1 / 3}$ ) could lead to the associated reduction in the effective central charge. The existence of towers of null states at the critical dimension depends on the structure of the chiral algebra of the $\tilde{G}_{r}$ 's. The form of this world-sheet symmetry algebra is, of course, one of the main mysteries of fractional superstrings. Some speculations on what this algebra may be will be discussed in Sect. 7.1.

Note that the resolution described above gives up manifest Lorentz covariance, and only demands covariance in the final step - the equations of motion. It is possible that a manifestly covariant formulation of the constraint algebra and states requres the introduction of the BRST ghost system, as in the fermionic sector of the usual superstring.

Another possible resolution aims at preserving manifest Lorentz covariance at all stages. Referring back to the first massive fermionic level calculation, we see that the non-covariance first appeared in the mode GCRs (6.4). One could try to maintain manifest covariance by modifying the commutation relations for $\mu \neq \nu$. This means that we are no longer simply taking the direct tensor product of $\mathbf{Z}_{4}$ parafermion theories. Instead, we are "deforming" this tensor product to obtain a new CFT. Since we are changing the GCRs among the modes, we are effectively changing the Fock space.

This program can be made systematic in the following way. Working level by level in the Fock space, one covariantizes the GCRs of the modes, which leads in general to the introduction of many free parameters. These parameters are fixed by then demanding that the resulting GCRs are associative (a necessary condition for them to describe a consistent two-dimensional field theory). One then has to check that this new Fock space provides a representation of the Virasoro algebra, as well as construct the new fractional supercurrent $\tilde{G}$.

The deformed tensor product approach is in general much harder to implement than the previous idea of changing the physical state conditions. There are many reasons for this. One reason is that in the former approach one has to solve the associativity conditions at each mass level. Another is that since the Fock space has been changed, a comparison to the partition function can only be made after solving the physical state conditions and removing the null states at each mass level. Note, 
however, that the two approaches outlined above may in principle be equivalent: the extra "physical state condition" which implements the internal projection on the original Fock space could be a weak operator realization of the tensor product deformation which reduces the Fock space to a manifestly covariant one. Indeed, one expects such a picture to hold if the deformed tensor product approach is to reproduce the partition function derived in Appendix D.

It is entirely possible that the use of the tensor-product Fock space as a starting point for the understanding of the $K=4$ FSS is incorrect. There are other possibilities, to which we will now turn.

\section{$7 \quad$ Remarks}

In this section we describe two features of the $K=4$ FSS which, though not directly related to the open string physical state condition calculations described above, may nevertheless play a role in the ultimate solution to the problems raised in the previous section.

\subsection{Connection to the spin- $4 / 3$ string}

A crucial issue in the understanding of the FSS is the determination of its critical space-time dimension $D$. We described in Appendix D an argument that determines the $K=4 \mathrm{FSS}$ critical dimension to be $D=6$. However, this argument is indirect, in that it does not determine $D$ from the consistency condition (anomaly cancellation) for the world-sheet gauge invariance. By solving the physical state conditions we can in principle check this determination of $D$-it should be the largest dimension in which a no-ghost theorem holds. This critical dimension is signalled by the presence of extra towers of null states in the spectrum. Because the generalized commutator algebra (3.9) for the components of the fractional supercurrent $G$ on the tensorproduct Fock space cannot be combined into a single algebra for the modes of $G$ itself, we can not construct these null state towers.

In light of the discussion of Sect. 6.3, however, we should consider modifying the algebra of physical state conditions. A particularly simple algebra is the onecomponent spin- $4 / 3$ algebra (3.3), but with arbitrary central charge [17],

$$
\begin{aligned}
G^{+}(z) G^{+}(w) & =\frac{\lambda(c)}{(z-w)^{4 / 3}}\left\{G^{-}(w)+\frac{1}{2}(z-w) \partial G^{-}(w)\right\}, \\
G^{-}(z) G^{-}(w) & =\frac{\lambda(c)}{(z-w)^{4 / 3}}\left\{G^{+}(w)+\frac{1}{2}(z-w) \partial G^{+}(w)\right\}, \\
G^{+}(z) G^{-}(w) & =\frac{(1 / 2)}{(z-w)^{8 / 3}}\left\{\frac{3 c}{4}+2(z-w)^{2} T(w)\right\}, \\
T(z) G^{ \pm}(w) & =\frac{(4 / 3) G^{ \pm}(w)}{(z-w)^{2}}+\frac{\partial G^{ \pm}(w)}{(z-w)}
\end{aligned}
$$




$$
T(z) T(w)=\frac{(c / 2)}{(z-w)^{4}}+\frac{2 T(w)}{(z-w)^{2}}+\frac{\partial T(w)}{(z-w)} .
$$

The structure constant $\lambda(c)$ is fixed by the condition of associativity of this algebra (see Appendix C):

$$
\lambda^{2}(c)=\frac{8-c}{6} .
$$

We will call the hypothetical string theory with (7.1) as its constraint algebra a "spin-4/3 string."

A straightforward calculation, presented in Appendix E, shows that the spin- $4 / 3$ string has towers of extra null states at the critical central charge $c=10$. This result was obtained earlier by an examination of the Kač determinant formula [\$]. This value of $c$ is different from the central charge $c=2 D=12$ of the $K=4$ FSS discussed above. However, as mentioned in Sect. 6.2, the effective central charge for the light-cone degrees of freedom of the $K=4 \mathrm{FSS}$ is $c_{\text {eff }}=6$ by the partition function, instead of the expected $c_{\text {eff }}=8$. This suggests that the correct covariant space of states might be a $c=10$ representation of the spin- $4 / 3$ string algebra (7.1), instead of the $c=12$ tensor product representation of the FSS constraint algebra (3.5).

Indeed, some numerical evidence can be adduced to support this supposition. As mentioned in the Introduction, the general- $K$ FSS has critical space-time dimension

$$
D=2+\frac{16}{K},
$$

and the central charge $c_{0}$ per dimension is thought to correspond to that of a $\mathbf{Z}_{K}$ parafermion theory plus a free coordinate boson; i.e.

$$
c_{0}=\frac{2(K-1)}{K+2}+1=\frac{3 K}{K+2} .
$$

On the other hand, the effective central charge for the light-cone degrees of freedom (from the FSS partition functions) is [13]

$$
c_{\text {eff }}=(D-2) \cdot \frac{3}{2} .
$$

Thus we could expect the total critical central charge to be

$$
c=c_{\mathrm{eff}}+2 c_{0}=\frac{6 K}{K+2}+\frac{24}{K} .
$$

But these $c$ are precisely the critical central charges obtained in ref. [8] for the spin$(K+4) /(K+2)$ strings, by demanding towers of extra null states. In particular, when $K=4$ we find $c=10$, corresponding to the spin- $4 / 3$ case calculated in Appendix E. 
Unfortunately, the representations of the spin-4/3 algebra at $c=10$ are not well understood, and in particular no representation with a flat target space-time interpretation is known. Thus, a direct construction of the spin- $4 / 3$ string and comparison to the $K=4 \mathrm{FSS}$ partition function is not yet possible. However, it is possible that string theories with fractional world-sheet supersymmetry embedded in curved backgrounds can be constructed, and may prove interesting to study. A suggestive example of an affine Lie algebra whose Wess-Zumino-Witten theory has central charge $c=10$ and has the correct field content to construct a dimension- $4 / 3$ group-invariant current is $S O(5,1)_{8}$. This conformal field theory is thus a candidate representation of the spin- $4 / 3$ string constraint algebra (7.1) at $c=10$.

We should point out that the $c=12$ FSS may be compatible with the $c=10$ spin- $4 / 3$ string in the following sense. Partial gauge-fixing of the $c=12$ FSS may reduce to a $c=10$ theory with the spin- $4 / 3$ algebra as the remaining constraint algebra.

\subsection{Chirality and anomalies in the fractional superstring}

In the preceding sections, we discussed the massless spectrum of the open $K=4$ FSS, which contains a minimal Yang-Mills supermultiplet $\left(A_{\mu}, \psi^{L}\right)$, where $A_{\mu}$ is a vector field and $\psi^{L}$ is a left-handed Weyl spinor field in six dimensions. The massless spectrum described by the partition function of the closed FSS follows in a simple way from that of the open string theory. In the closed FSS there are supermultiplets corresponding to both the left-moving and righ-moving sectors, so to obtain the spectrum we must take the tensor product of these two supermultiplets. There are two possibilities for doing this, which we will call Type IIA and Type IIB, in analogy to the ten-dimensional superstring. In the Type IIA model, the two supermultiplets are chosen so that the corresponding spinor fields are of opposite chirality. Taking the tensor product, we see that the Type IIA closed FSS contains an $N=2$ supergravity multiplet:

$$
\begin{gathered}
\left(A_{\mu}, \psi^{L}\right) \otimes\left(A_{\mu}, \psi^{R}\right)=g_{\mu \nu}+B_{\mu \nu}^{L}+B_{\mu \nu}^{R} \\
+\psi_{\mu}^{L}+\psi_{\nu}^{R}+4 A_{\mu}+\psi^{L}+\psi^{R}+\phi
\end{gathered}
$$

where $g_{\mu \nu}$ is the graviton, $B_{\mu \nu}$ are (anti-)self-dual antisymmetric tensor fields, $\psi_{\mu}$ is the gravitino, $\psi$ is a spinor field, and $\phi$ is a scalar field. $L$ and $R$ denote left- and right-handedness, respectively. This spectrum clearly shows that the Type IIA closed FSS is non-chiral, exactly as in the ten-dimensional $(K=2)$ case.

The second possibility, Type IIB, is realized by choosing spinors of the same chirality in the two supermultiplets. In the ten-dimensional superstring, the spectra of the two types of closed superstrings are different, but the counting of states in each model is the same and they therefore have the same partition function. In addition, both models yield low-energy effective theories which are free of gravitational anomalies. 
Let us naively try to obtain the chiral supergravity multiplet for the Type IIB FSS:

$$
\left(A_{\mu}, \psi^{L}\right) \otimes\left(A_{\mu}, \psi^{L}\right)=g_{\mu \nu}+5 B_{\mu \nu}^{L}+B_{\mu \nu}^{R}+2 \psi_{\mu}^{L}+2 \psi^{R}+5 \phi .
$$

We will refer to this chiral $N=2$ multiplet as the $\alpha$ multiplet. It is easy to check that the resulting six-dimensional effective low-energy theory containing the $\alpha$ multiplet has gravitational anomalies. To cancel the anomalies, we may try to extend the theory by adding additional multiplets to the $\alpha$ multiplet. There are some constraints that must be satisfied. Since there is only one modular invariant partition function for the $K=4$ closed FSS (without any compactification), $\mathcal{Z}$ in eq. (D.10), the only possible way to accommodate additional massless states in the spectrum is to multiply $\mathcal{Z}$ by an integer; this increases the number of massless states by this integral factor. We do not want to increase the number of gravitons or gravitinos in the theory, however, so we cannot obtain these states from tensoring extra vector supermultiplets $\left(A_{\mu}, \psi^{L(R)}\right)$. The only other option is to form these extra multiplets, to be called $\beta$ multiplets, from tensoring chiral (scalar) supermultiplets $\left(\psi^{L(R)}, 4 \phi\right)$ :

$$
\left(\psi^{R}, 4 \phi\right) \otimes\left(\psi^{R}, 4 \phi\right)=4 B_{\mu \nu}^{R}+8 \psi^{R}+20 \phi .
$$

One can easily verify that the only anomaly-free chiral supergravity multiplet is given by adding five copies of the $\beta$ multiplet to the $\alpha$ multiplet, which yields the particle content

$$
g_{\mu \nu}+5 B_{\mu \nu}^{L}+2 \psi_{\mu}^{L}+21 B_{\mu \nu}^{R}+42 \psi^{R}+105 \phi .
$$

This six-dimensional chiral supergravity multiplet was first constructed in the $K_{3}$ compactification of the usual superstring [20]. The number of massless states in this chiral model is exactly six times that of the non-chiral model. Thus this counting of states implies that the partition function for the chiral model is

$$
\mathcal{Z}(\text { chiral })=6 \mathcal{Z}(\text { non }- \text { chiral })
$$

To obtain five $\beta$ multiplets in this model, the left- and right-moving sectors must each have at least three chiral multiplets. How can this theory account for all these extra states? One possibility can be found by recalling that there is more than one possible realization of the $\mathbf{Z}_{4}$ parafermion theory. In the bosonization given in Sect. 2, we see that each parafermion field is realized by two fields $\epsilon^{( \pm a)}$ with the same conformal dimension. This splitting mechanism can be carried out further with the introduction of cocycle operators, thus increasing the number of copies of a particular field. In Appendix A, we discuss the introduction of cocycle operators in the parafermion theory.

It is clear how this construction can increase the number of fermion fields in the model, by choosing a cocycle subalgebra which increases the number of spin fields $\sigma_{2}^{\mu}$, for example. However, the origin of the scalar fields which are the superpartners of these additional fermions is harder to ascertain, since we did not have scalar 
fields originally in the spectrum of the open string FSS. One possible solution to this problem is to reintroduce $\sigma_{ \pm 1}$, the $\mathbf{Z}_{4}$ parafermion spin fields of dimension $1 / 16$, mentioned in Sect. 2. States in the sector generated by these fields did not appear to contribute to the partition functions given in Sect. 3. However, if we consider the fusion rule $\left[\sigma_{1}\right]\left[\sigma_{-1}\right] \sim[\mathbb{1}]+[\epsilon]$, we see that the $\epsilon$ field appears. In the tensor product theory, the fields $\sigma_{ \pm 1}$ acquire a vector index $\mu$, and thus we may be able to regard some of the $\epsilon$ fields as Lorentz scalar composites of these spin fields, in a manner similar to the way that $\sum_{\mu} \epsilon^{( \pm 2), \mu}$ is realized as a scalar composite of $\epsilon^{( \pm 1), \mu}$ 's in eq. (3.17). In this way we may be able to obtain the required scalars.

\section{Acknowledgments}

It is a pleasure to thank K. Dienes, J. Grochocinski and J. Schwarz for useful discussions and comments. This work was supported in part by the National Science Foundation.

\section{Appendix A}

In this appendix we discuss representations of the $\mathbf{Z}_{4}$ parafermion theory (or $S U(2)_{4} / U(1)$ coset theory) that can be constructed in terms of cocycles and the free boson $\rho(z)$ introduced in Sect. 2. These alternative representations could, in principle, be the correct building blocks for the $K=4$ FSS Fock space, instead of the free boson representation discussed in the body of this paper. For this reason it is important to explore the space of inequivalent representations of the $\mathbf{Z}_{4}$ parafermion theory.

Restricting ourselves just to those that can be constructed with a free boson plus cocycles, there is already an infinite number of representations. By further restricting our inquiries by ad hoc simplifying assumptions, we will construct a few inequivalent representations, and point out their main properties. In particular, some of these representations have structure constants for their operator algebra different from those of the free boson representation. In other representations the OPEs no longer

satisfy abelian braiding relations as (2.11)-(2.13) do. This makes the physical state conditions technically more difficult to implement. It is for these reasons that the discussion in the body of the paper has been limited to the free boson representation without cocycles.

Since the free boson $\rho(z)$ CFT is associative by itself, any cocycles that are attached to it must also be associative if the combined theory is to be. Thus, we expect the cocycles to form a finite-dimensional associative algebra including the identity. Inequivalent examples of such cocycle algebras are the algebras of $n \times n$ matrices with real, complex or quaternionic entries. By taking the direct product of these 
algebras with the free boson OPE algebra, we obtain an infinite number of inequivalent representations of the $\mathbf{Z}_{4}$ parafermion theory. In the construction that follows we will restrict ourselves to the simplest cocycle algebras: $1 \times 1$ real matrices (i.e. no cocycles) and $2 \times 2$ real matrices.

We start with the free boson representation without cocycles used in the body of this paper. By comparing the OPEs (2.11)-(2.13) of the free boson primary fields to the parafermion fusion rules (2.4) we can identify which primary fields belong to each parafermion sector $\left[\phi_{m}^{j}\right]$ :

$$
\begin{array}{ll}
{\left[\phi_{0}^{0}\right]_{0}=\left\{\epsilon^{(3 n)}+\epsilon^{(-3 n)}, n \geq 0\right\},} & {\left[\phi_{0}^{2}\right]_{0}=\left\{i \partial \rho ; \epsilon^{(3 n)}-\epsilon^{(-3 n)}, n>0\right\},} \\
{\left[\phi_{1}^{1}\right]_{0}=\left\{\epsilon^{(3 n+1 / 2)}\right\},} & {\left[\phi_{1}^{1}\right]_{0}^{\prime}=\left\{\epsilon^{(3 n-1 / 2)}\right\},} \\
{\left[\phi_{0}^{1}\right]_{0}=\left\{\epsilon^{(3 n+1)}\right\},} & {\left[\phi_{0}^{1}\right]_{0}^{\prime}=\left\{\epsilon^{(3 n-1)}\right\},} \\
{\left[\phi_{1}^{0}\right]_{0}+\left[\phi_{-1}^{0}\right]_{0}=\left\{\epsilon^{(3 n+3 / 2)}\right\},} &
\end{array}
$$

where $n$ runs over the integers in the specified ranges. The subscript zero is to identify the representation. Note that the $\left[\phi_{0}^{1}\right]$ and $\left[\phi_{1}^{1}\right]$ sectors each appear twice in this representation of the parafermion theory. This is clear from the identifications of the energy operator $\epsilon$ and the spin field $\sigma_{2}$ in $(2.10)$, where they each have multiplicity two: $\epsilon$ can be represented by either $\epsilon^{(+1)}$ or $\epsilon^{(-1)}$, and $\sigma_{2}$ by either $\epsilon^{(+1 / 2)}$ or $\epsilon^{(-1 / 2)}$. Also, the parafermion current sectors $\left[\phi_{ \pm 1}^{0}\right]$ cannot be separated in a way consistent with the fusion rules (2.4). This property implies that this $\mathbf{Z}_{4}$ parafermion representation is not really a faithful representation of the $S U(2)_{4}$ fusion rules (2.4). This is a reflection of the fact that in (2.11) $-(2.13)$ only a single cut occurs on the right hand side of any given OPE. This situation is called abelian (or sometimes parafermionic) braiding.

We can form a representation with single multiplicity for the $\left[\phi_{0}^{1}\right]$ and $\left[\phi_{1}^{1}\right]$ sectors by considering only the symmetric subalgebra of the 0-representation (A.1):

$$
\begin{aligned}
& {\left[\phi_{0}^{0}\right]_{1}=\left\{\epsilon^{(3 n)}+\epsilon^{(-3 n)}, n \geq 0\right\},} \\
& {\left[\phi_{1}^{1}\right]_{1}=\left\{\epsilon^{(3 n+1 / 2)}+\epsilon^{(-3 n-1 / 2)}\right\},} \\
& {\left[\phi_{0}^{1}\right]_{1}=\left\{\epsilon^{(3 n+1)}+\epsilon^{(-3 n-1)}\right\},} \\
& {\left[\phi_{1}^{0}\right]_{1}=\left\{\epsilon^{(3 n+3 / 2)}+\epsilon^{(-3 n-3 / 2)}, n \geq 0\right\} .}
\end{aligned}
$$

This representation satisfies non-abelian braiding relations. In particular, upon braiding the fields in this representation we introduce new fields (on the next Riemann sheet). The set of all fields on all three sheets just reproduces the field content of representation-0, though the field content on each sheet separately is that given in (A.2). Representation-1 has single energy and spin-field multiplicity, but has zero spin-2 multiplicity (i.e. the $\left[\phi_{0}^{2}\right]$ sector does not appear). This latter property implies that representation-1 is no good for FSS building since the arguments of Appendix 
D show that the spin-2 parafermion fields enter into the FSS spectrum. Note that this representation is a faithful representation of the $S U(2)_{4}$ fusion rules (with only spin-0 and spin-1 fields) but, since the spin-2 fields decouple, cannot realize the $\mathbf{Z}_{4}$ parafermion current algebra, and therefore also can not realize the $S U(2)_{4} \mathrm{Kac}-$ Moody current algebra via eq. (2.5).

The algebra of $2 \times 2$ real matrices is generated by the four elements:

$$
\boldsymbol{\alpha}=\left(\begin{array}{ll}
0 & 1 \\
0 & 0
\end{array}\right), \quad \boldsymbol{\beta}=\left(\begin{array}{ll}
0 & 0 \\
1 & 0
\end{array}\right), \quad \boldsymbol{\gamma}=\left(\begin{array}{cc}
1 & 0 \\
0 & -1
\end{array}\right), \quad \mathbf{1}=\left(\begin{array}{ll}
1 & 0 \\
0 & 1
\end{array}\right)
$$

which satisfy the algebra

$$
\begin{aligned}
\boldsymbol{\alpha}^{2}=\boldsymbol{\beta}^{2}=0, & \boldsymbol{\gamma}^{2}=\mathbf{1}, \\
2 \boldsymbol{\alpha} \boldsymbol{\beta}=\mathbf{1}+\boldsymbol{\gamma}, & 2 \boldsymbol{\beta} \boldsymbol{\alpha}=\mathbf{1}-\boldsymbol{\gamma}, \\
\boldsymbol{\beta} \gamma=-\boldsymbol{\gamma} \boldsymbol{\gamma}=\boldsymbol{\beta}, & \boldsymbol{\gamma} \boldsymbol{\alpha}=-\boldsymbol{\alpha} \boldsymbol{\gamma}=\boldsymbol{\alpha} .
\end{aligned}
$$

If we take a direct tensor product of the $c=1$ free boson theory with this set of cocycles, we simply obtain a new $c=1$ theory with four times as many fields. The point, however, is that this new theory has closed subalgebras which could not be realized in the free boson theory alone. We will construct two inequivalent such representations of the $\mathbf{Z}_{4}$ parafermion sectors with multiplicities less than or equal to those of the 0-representation.

The first is

$$
\begin{aligned}
{\left[\phi_{0}^{0}\right]_{2} } & =\left\{\boldsymbol{1}\left(\epsilon^{(3 n)}+\epsilon^{(-3 n)}\right), n \geq 0\right\}, \\
{\left[\phi_{0}^{2}\right]_{2} } & =\left\{\boldsymbol{\gamma}(i \partial \rho) ; \boldsymbol{\gamma}\left(\epsilon^{(3 n)}-\epsilon^{(-3 n)}\right), n>0\right\} \\
{\left[\phi_{1}^{1}\right]_{2} } & =\left\{\boldsymbol{\beta} \epsilon^{(3 n+1 / 2)}+\boldsymbol{\alpha} \epsilon^{(-3 n-1 / 2)}\right\}, \\
{\left[\phi_{0}^{1}\right]_{2} } & =\left\{\boldsymbol{\alpha} \epsilon^{(3 n+1)}+\boldsymbol{\beta} \epsilon^{(-3 n-1)}\right\}, \\
{\left[\phi_{1}^{0}\right]_{2} } & =\left\{\boldsymbol{1}\left(\epsilon^{(3 n+3 / 2)}+\epsilon^{(-3 n-3 / 2)}\right), n \geq 0\right\}, \\
{\left[\phi_{-1}^{0}\right]_{2} } & =\left\{\boldsymbol{\gamma}\left(\epsilon^{(3 n+3 / 2)}-\epsilon^{(-3 n-3 / 2)}\right), n \geq 0\right\} .
\end{aligned}
$$

It is easily checked that this set of fields forms a closed algebra under fusion. This representation is also non-abelianly braided, but has single multiplicities, and is a faithful representation of the $S U(2)_{4}$ fusion rules (with all spins). However, it has no $\epsilon \sigma_{2} \sigma_{2}=c_{111}$ coupling (i.e. no coupling between the $\left[\phi_{0}^{1}\right]$ and two $\left[\phi_{1}^{1}\right]$ sectors), which implies that representation-2 is not suitable for FSS building, since, as will be shown in Sect. 1, this coupling is crucial for the description of space-time spinors in the FSS Fock space. Note that the spin- 2 sector OPEs (involving the $\left[\phi_{0}^{2}\right]$ and $\left[\phi_{ \pm 1}^{0}\right]$ fields) do not have a $\mathbf{Z}_{4}$ symmetry and thus do not form a representation of the parafermion current algebra. 
It is easiest to write the second representation with cocycles in terms of the basis $\{\mathbf{1}, \mathbf{i}, \mathbf{j}, \mathbf{k}\}$ where $\mathbf{i} \equiv-\boldsymbol{\alpha}-\boldsymbol{\beta}, \mathbf{j}=\boldsymbol{\gamma}$ and $\mathbf{k}=\boldsymbol{\alpha}-\boldsymbol{\beta}$. They satisfy the relations

$$
\mathbf{i}^{2}=\mathbf{j}^{2}=-\mathbf{k}^{2}=\mathbf{1}, \quad \mathbf{i j}=-\mathbf{j i}=\mathbf{k}, \quad \mathbf{j k}=-\mathbf{k j}=-\mathbf{i}, \quad \mathbf{k i}=-\mathbf{i k}=-\mathbf{j} .
$$

A closed algebra is composed of the sectors

$$
\begin{aligned}
{\left[\phi_{0}^{0}\right]_{3} } & =\left\{\mathbf{k}^{n} \epsilon^{(3 n)}+\mathbf{k}^{-n} \epsilon^{(-3 n)}, n \geq 0\right\} \\
{\left[\phi_{0}^{2}\right]_{3} } & =\left\{\mathbf{k}(i \partial \rho) ; \mathbf{k}^{n+1} \epsilon^{(3 n)}+\mathbf{k}^{-n-1} \epsilon^{(-3 n)}, n>0\right\} \\
{\left[\phi_{1}^{1}\right]_{3} } & =\left\{\mathbf{k}^{n} \mathbf{i} \epsilon^{(3 n+1 / 2)}+\mathbf{k}^{-n} \mathbf{j} \epsilon^{(-3 n-1 / 2)}\right\} \\
{\left[\phi_{1}^{1}\right]_{3}^{\prime} } & =\left\{\mathbf{k}^{n} \mathbf{j} \epsilon^{(3 n+1 / 2)}+\mathbf{k}^{-n} \mathbf{i} \epsilon^{(-3 n-1 / 2)}\right\} \\
{\left[\phi_{0}^{1}\right]_{3} } & =\left\{\mathbf{k}^{n} \epsilon^{(3 n+1)}+\mathbf{k}^{-n} \epsilon^{(-3 n-1)}\right\} \\
{\left[\phi_{0}^{1}\right]_{3}^{\prime} } & =\left\{\mathbf{k}^{n+1} \epsilon^{(3 n+1)}+\mathbf{k}^{-n-1} \epsilon^{(-3 n-1)}\right\}, \\
{\left[\phi_{1}^{0}\right]_{3} } & =\left\{\mathbf{k}^{n} \mathbf{i} \epsilon^{(3 n+3 / 2)}+\mathbf{k}^{-n} \mathbf{j} \epsilon^{(-3 n-3 / 2)}, n \geq 0\right\} \\
{\left[\phi_{-1}^{0}\right]_{3} } & =\left\{\mathbf{k}^{n} \mathbf{j} \epsilon^{(3 n+3 / 2)}+\mathbf{k}^{-n} \mathbf{i} \epsilon^{(-3 n-3 / 2)}, n \geq 0\right\}
\end{aligned}
$$

Note that $\mathbf{k}^{-1}=-\mathbf{k}$. This representation is non-abelianly braided in general but has a $\mathbf{Z}_{4}$ symmetry in its parafermion current sector. Thus the parafermion fields $\phi_{0}^{0}, \phi_{ \pm 1}^{0}$ and $\phi_{0}^{2}$ form an abelianly braided subalgebra. Of the four $\mathbf{Z}_{4}$ parafermion representations presented in this Appendix, this is the only one to have the defining $\mathbf{Z}_{4}$ symmetry, and, consequently, the only one which allows a realization of the $S U(2)_{4}$ current algebra by adding a boson. Note that this representation has the same multiplicities as the free boson representation (representation-0), and forms a faithful representation of the $S U(2)_{4}$ fusion rules (2.4) with double spin-1 multiplicities. $A$ priori, representation-3 is just as good as representation-0 for building the FSS Fock space. In practice, it has the technical disadvantage that the $\mathbf{Z}_{4}$ symmetry only extends to part of its spectrum, unlike the free boson case, where its $\mathbf{Z}_{3}$ symmetry includes all its fields. This $\mathbf{Z}_{3}$ symmetry is a major simplifing feature of the analysis presented in the body of this paper.

There exist many more representations that can be found by taking larger cocycle spaces. It is possible that some of these other representations have subalgebras with low multiplicities which are not equivalent to representations 0-3 constructed above. Also, one can build other, essentially free, representations of the $\mathbf{Z}_{4}$ parafermions by orbifolding the free boson $\rho(z)$ of the above representations. It is found [15] that the twist fields introduced by a $\mathbf{Z}_{2}$ orbifolding correspond to the half-odd integer spin sectors of the parafermion theory, which include the dimension- $1 / 16$ spin-fields. We have not considered orbifolds in this paper because the arguments of Appendix D show that the half-odd spin sectors do not contribute to the FSS partition function. See however the discussion of chiral fermions in Sect. 7.2 where these twist fields may play a role. 


\section{Appendix B}

In this appendix we will review the argument of Zamolodchikov and Fateev [3] which leads to the derivation of generalized commutation relations (GCRs) for abelianly braided operators. We will do this by an example: we derive the GCRs for the $\mathbf{Z}_{4}$ parafermion fields $\epsilon^{(+1)}$ and $\epsilon^{(-1)}$. The general procedure should be clear from this example.

The $\mathbf{Z}_{4}$ parafermion Fock space falls into sectors $\mathcal{H}_{q}$ labelled by their $\mathbf{Z}_{3}$ charge $q$. The dimension- $1 / 3$ energy operators $\epsilon^{(+1)}$ and $\epsilon^{(-1)}$ carry $\mathbf{Z}_{3}$ charges $q=+1$ and -1 , respectively. To simplify notation, we will denote $\epsilon^{(+1)}$ by $\epsilon$ and $\epsilon^{(-1)}$ by $\epsilon^{\dagger}$. They act on the Fock space by the rule

$$
\epsilon: \mathcal{H}_{q} \rightarrow \mathcal{H}_{q+1}, \quad \epsilon^{\dagger}: \mathcal{H}_{q} \rightarrow \mathcal{H}_{q-1}
$$

where the $\mathbf{Z}_{3}$ charge is defined mod 3. Their mode expansions are defined, as in eq. (2.25), by

$$
\begin{aligned}
\epsilon(z) \chi_{q}(0) & =\sum_{n} z^{n-\frac{q}{3}} \epsilon_{-n+(q-1) / 3} \chi_{q}(0), \\
\epsilon^{\dagger}(z) \chi_{q}(0) & =\sum_{n} z^{n+\frac{q}{3}} \epsilon_{-n-(q+1) / 3}^{\dagger} \chi_{q}(0)
\end{aligned}
$$

where $\chi_{q}$ is an arbitrary state in $\mathcal{H}_{q}$. The mode expansions can be inverted to give

$$
\begin{aligned}
\epsilon_{n+(q-1) / 3} \chi_{q}(0) & =\oint_{\gamma} \frac{\mathrm{d} z}{2 \pi i} z^{n-1+q / 3} \epsilon(z) \chi_{q}(0) \\
\epsilon_{n-(q+1) / 3}^{\dagger} \chi_{q}(0) & =\oint_{\gamma} \frac{\mathrm{d} z}{2 \pi i} z^{n-1-q / 3} \epsilon^{\dagger}(z) \chi_{q}(0)
\end{aligned}
$$

where $\gamma$ is a contour encircling the origin once, where $\chi_{q}(0)$ is inserted.

The OPE of $\epsilon$ with $\epsilon^{\dagger}$ is given by [see eq. (2.12)]

$$
\epsilon(z) \epsilon^{\dagger}(w)=\frac{1}{(z-w)^{2 / 3}}+(z-w)^{1 / 3} i \partial \rho(w)+\ldots
$$

Now we will derive the generalized commutation relations that the $\epsilon$ and $\epsilon^{\dagger}$ modes satisfy as a result of this OPE. Consider the integral

$$
\mathcal{I}=\oint_{\gamma} \frac{\mathrm{d} z}{2 \pi i} \oint_{\delta} \frac{\mathrm{d} w}{2 \pi i} z^{m-1+q / 3} w^{n-1-q / 3}(z-w)^{p+2 / 3} \epsilon(z) \epsilon^{\dagger}(w) \chi_{q}(0)
$$

where $m, n$ and $p$ are arbitrary integers. The contour $\gamma$ encircles $\delta$, which in turn encircles the origin. The the fractional parts of the exponents in the integrand are chosen so that the whole integrand is single-valued in both the $z$ - and $w$-planes. This is possible only because of the abelian nature of the $\epsilon \epsilon^{\dagger} \mathrm{OPE}$ (B.4). We can evaluate 
this integral by letting $\delta$ shrink down to a small circle near to the origin. In this limit we can expand the $(z-w)^{p+2 / 3}$ factor as

$$
(z-w)^{p+2 / 3}=z^{p+2 / 3} \sum_{\ell=0}^{\infty} c_{\ell}^{(p+2 / 3)}\left(\frac{w}{z}\right)^{\ell},
$$

where $c_{\ell}^{(\alpha)}$ are the appropriate binomial coefficients. Inserting this expansion into eq. (B.5), and using the mode definitions $(\mathbb{B} .3)$, we find

$$
\mathcal{I}=\sum_{\ell=0}^{\infty} c_{\ell}^{(p+2 / 3)} \epsilon_{m+p-\ell+\frac{q+1}{3}} \epsilon_{n+\ell-\frac{q+1}{3}}^{\dagger} \chi_{q}(0)
$$

$\mathcal{I}$ can also be evaluated in another way, by first deforming the $\gamma$ contour so that it lies inside $\delta$. Upon performing this deformation, one picks up in the usual way two contributions

$$
\mathcal{I}=\mathcal{I}^{\prime}+\mathcal{I}_{0}
$$

corresponding to the same integral $\mathcal{I}^{\prime}$ with $\gamma$ and $\delta$ interchanged, and the new contribution $\mathcal{I}_{0}$ where the $\gamma$ contour encircles the $\epsilon^{\dagger}$ insertion at the point $w$ on the $z$-plane. $\mathcal{I}^{\prime}$ can be evaluated in the same way as $\mathcal{I}$ was, after interchanging $\epsilon(z)$ and $\epsilon^{\dagger}(w)$ as well as $z$ and $w$ in the $(z-w)^{p+2 / 3}$ factor. Taking care to perform these interchanges along equivalent paths in the complex plane gives an overall phase $\mathrm{e}^{i \pi(-2 / 3)} \times \mathrm{e}^{i \pi(p+2 / 3)}=(-1)^{p}$. Thus

$$
\begin{aligned}
\mathcal{I}^{\prime} & =(-1)^{p} \oint_{\delta^{\prime}} \frac{\mathrm{d} z}{2 \pi i} \oint_{\gamma^{\prime}} \frac{\mathrm{d} w}{2 \pi i} z^{m-1+q / 3} w^{n-1-q / 3}(w-z)^{p+2 / 3} \epsilon^{\dagger}(w) \epsilon(z) \chi_{q}(0) \\
& =(-1)^{p} \sum_{\ell=0}^{\infty} c_{\ell}^{(p+2 / 3)} \epsilon_{n+p-\ell-\frac{q-1}{3}}^{\dagger} \epsilon_{m+\ell+\frac{q-1}{3}} \chi_{q}(0)
\end{aligned}
$$

where we have again used the mode definitions (B.3). Note that the abelian braid property of the $\epsilon \epsilon^{\dagger} \mathrm{OPE}$ was important in performing the analytic continuation needed to define the integrand of $\mathcal{I}^{\prime}$. Because only one kind of cut appears in (B.4), under the analytic continuation which interchanges $z$ and $w, \epsilon(z) \epsilon^{\dagger}(w)$ only gains a simple phase. Indeed, this property can be taken as the definition of abelian braiding.

The contribution $\mathcal{I}_{0}$ is the same as $\mathcal{I}$ except that $\gamma$, instead of circling the origin, now only encircles the point $w$ in the $z$-plane. Letting this contour shrink to a small circle around $w$, we can replace $\epsilon(z) \epsilon^{\dagger}(w)$ by their OPE (B.4). The value of the integer $p$ in the integrand controls the number of terms in the OPE that contribute. For example, taking $p=-1$ gives

$$
\begin{aligned}
\mathcal{I}_{0} & =\oint_{0} \frac{\mathrm{d} w}{2 \pi i} w^{n-1-q / 3} \oint_{w} \frac{\mathrm{d} z}{2 \pi i} z^{m-1+q / 3}\left\{(z-w)^{-1}+\ldots\right\} \chi_{q}(0) \\
& =\oint \frac{\mathrm{d} w}{2 \pi i} w^{n+m-2} \chi_{q}(0)=\delta_{n+m-1} .
\end{aligned}
$$


These expressions for $\mathcal{I}, \mathcal{I}^{\prime}$ and $\mathcal{I}_{0}$ can be combined according to eq. (B.8) to give a generalized commutation relation for the $\epsilon$ and $\epsilon^{\dagger}$ modes:

$$
\sum_{\ell=0}^{\infty} c_{\ell}^{(-1 / 3)}\left[\epsilon_{m-1-\ell+\frac{q+1}{3}} \epsilon_{n+\ell-\frac{q+1}{3}}^{\dagger}+\epsilon_{n-1-\ell-\frac{q-1}{3}}^{\dagger} \epsilon_{m+\ell+\frac{q-1}{3}}\right]=\delta_{n+m-1},
$$

understood to be acting on any state $\chi_{q} \in \mathcal{H}_{q}$. Alternatively we could have chosen $p=-2$, which would pick up a contribution from the $\partial \rho(w)$ term in the $\epsilon \epsilon^{\dagger} \mathrm{OPE}$, to give the GCR

$$
\begin{gathered}
\sum_{\ell=0}^{\infty} c_{\ell}^{(-4 / 3)}\left[\epsilon_{m-2-\ell+\frac{q+1}{3}} \epsilon_{n+\ell-\frac{q+1}{3}}^{\dagger}-\epsilon_{n-2-\ell-\frac{q-1}{3}}^{\dagger} \epsilon_{m+\ell+\frac{q-1}{3}}\right] \\
=\left(m-1+\frac{q}{3}\right) \delta_{n+m-1}+s_{n+m-2},
\end{gathered}
$$

where we have used the mode expansion of the $\rho(z)$ field in the form

$$
s_{m}=\oint \frac{\mathrm{d} z}{2 \pi i} i \partial \rho(z) z^{m} .
$$

It is clear that by letting $p$ take more negative values, more complicated GCRs involving more terms from the $\epsilon \epsilon^{\dagger}$ OPE can be obtained. By conformal invariance, this tower of GCRs is consistent. Indeed, the GCR obtained with $p=p_{0}$ can be derived from the GCR with $p=p_{0}-1$ using the binomial coefficient identity

$$
c_{\ell}^{(\alpha)}-c_{\ell-1}^{(\alpha)}=c_{\ell}^{(\alpha+1)}
$$

It should also be clear that the argument reviewed here works equally well for deriving GCRs from any abelianly braided OPE.

\section{Appendix $\mathrm{C}$}

In this appendix, we discuss the representation theory and associativity (or consistency) conditions of the fractional superconformal algebras. We begin with the split algebra (3.3). The explicit form for the currents of this algebra in the coordinate boson plus $\mathbf{Z}_{4}$ parafermion CFT is given in eq. (3.2). This CFT has central charge $c_{0}=2$. We can generalize the split algebra (3.3) to the case of a CFT with arbitrary central charge $c_{0}$ [17]:

$$
\begin{aligned}
G^{+}(z) G^{+}(w) & =\frac{\lambda\left(c_{0}\right)}{(z-w)^{4 / 3}}\left\{G^{-}(w)+\frac{1}{2}(z-w) \partial G^{-}(w)\right\}, \\
G^{-}(z) G^{-}(w) & =\frac{\lambda\left(c_{0}\right)}{(z-w)^{4 / 3}}\left\{G^{+}(w)+\frac{1}{2}(z-w) \partial G^{+}(w)\right\}, \\
G^{+}(z) G^{-}(w) & =\frac{(1 / 2)}{(z-w)^{8 / 3}}\left\{\frac{3 c_{0}}{4}+2(z-w)^{2} T(w)\right\},
\end{aligned}
$$


where we have only included terms on the right hand side with negative powers of $(z-w) . \lambda\left(c_{0}\right)$ is the undetermined structure constant of this algebra. (The other coefficients are determined by conformal invariance.) We found by explicit construction in Sect. 3 that $\lambda(2)=1$.

Because the operator algebra (C.1) is abelianly braided (that is, only a single cut appears on the right hand side of each $\mathrm{OPE}$ ), we can derive a Ward identity relating correlators with a $G^{+} G^{-}$pair to ones with the pair removed. Following Zamolodchikov and Fateev [3, 17], we can then solve for the structure constant $\lambda$ by imposing the associativity condition on the four-point function

$$
\mathcal{G}\left(z_{i}\right)=\left\langle G^{+}\left(z_{1}\right) G^{+}\left(z_{2}\right) G^{-}\left(z_{3}\right) G^{-}\left(z_{4}\right)\right\rangle .
$$

Actually, we will derive a simpler Ward identity which is valid only for the four-point function $\mathcal{G}$. Consider the function $\mathcal{F}\left(z_{i}\right)$ defined by

$$
\mathcal{F}\left(z_{i}\right)=\left(z_{1}-z_{2}\right)^{1 / 3}\left(z_{1}-z_{3}\right)^{2 / 3}\left(z_{1}-z_{4}\right)^{2 / 3} \mathcal{G}\left(z_{i}\right) .
$$

Because of the abelian nature of the spin- $4 / 3$ algebra, $\mathcal{F}$ is a holomorphic function of $z_{1}$ with a first-order pole at $z_{2}$ and second-order poles at $z_{3}$ and $z_{4}$. Also, since by conformal invariance $\mathcal{G} \sim z_{1}^{-8 / 3}$ as $z_{1} \rightarrow \infty$, in the same limit $\mathcal{F} \sim 1 / z_{1} \rightarrow 0$. Thus $\mathcal{F}$ is completely determined by the residues of its poles, which are easily read off from eq. (C.3) and the OPEs (C.1). .f The resulting Ward identity for $\mathcal{G}$ is

$$
\begin{aligned}
\mathcal{G}=( & \left.z_{1}-z_{2}\right)^{-1 / 3}\left(z_{1}-z_{3}\right)^{-2 / 3}\left(z_{1}-z_{4}\right)^{-2 / 3} \\
\times & \left\{\lambda\left(z_{1}-z_{2}\right)^{-1}\left(z_{2}-z_{3}\right)^{2 / 3}\left(z_{2}-z_{4}\right)^{2 / 3}\left\langle G^{-}\left(z_{2}\right) G^{-}\left(z_{3}\right) G^{-}\left(z_{4}\right)\right\rangle\right. \\
+ & \frac{c_{0}}{8}\left(z_{1}-z_{3}\right)^{-1}\left[3\left(z_{1}-z_{3}\right)^{-1}-\left(z_{2}-z_{3}\right)^{-1}+2\left(z_{3}-z_{4}\right)^{-1}\right] \\
& \quad \times\left(z_{2}-z_{3}\right)^{1 / 3}\left(z_{3}-z_{4}\right)^{2 / 3}\left\langle G^{+}\left(z_{2}\right) G^{-}\left(z_{4}\right)\right\rangle \\
+ & \frac{c_{0}}{8}\left(z_{1}-z_{4}\right)^{-1}\left[3\left(z_{1}-z_{4}\right)^{-1}-\left(z_{2}-z_{4}\right)^{-1}-2\left(z_{3}-z_{4}\right)^{-1}\right] \\
& \left.\times\left(z_{2}-z_{4}\right)^{1 / 3}\left(z_{3}-z_{4}\right)^{2 / 3}\left\langle G^{+}\left(z_{2}\right) G^{-}\left(z_{3}\right)\right\rangle\right\} .
\end{aligned}
$$

The remaining two- and three-point functions can be directly evaluated from the spin-4/3 OPEs to give a closed form expression for $\mathcal{G}$. Associativity can then be checked by taking, say, the limit as $z_{2} \rightarrow z_{3}$ in this expression, and checking that the residues of the poles match those determined by the OPEs. This fixes $\lambda$ as a function of $c_{0}$ :

$$
\lambda^{2}=\frac{8-c_{0}}{6}
$$

* This argument must be changed for higher-point functions to make $\mathcal{F}$ vanish as $z_{1} \rightarrow \infty$. In general, this requires the presence of third-order poles in $\mathcal{F}$ as $z_{1}$ approaches a $G^{-}$insertion. 
A crucial assumption that enabled us to integrate the Ward identity was that no fractional cuts not allowed by our abelian braiding assumption occur on the righthand side of the OPEs (C.1), even among the "regular" terms. We present an example below of how the associativity condition (C.5) may be modified by the inclusion of new cuts among the regular terms.

Much work has been done to understand the representation theory of this algebra (and related non-local algebras) [17, 5, 6, 0, 10, 11, 21, 22]. In particular, it is known that the split algebra (C.1), as well as the algebra formed from it by adding together $G^{+}$and $G^{-}$, have a series of unitary minimal representations. These can be realized by the coset models $S U(2)_{4} \otimes S U(2)_{L} / S U(2)_{L+4}$ with central charges

$$
c_{0}(L)=2-\frac{24}{(L+2)(L+6)} \quad \text { for } \quad L=1,2, \ldots
$$

Note that the central charge of these minimal models has an accumulation point at $c_{0}=2$, the central charge of the free CFT we are using in the construction of the FSS. This is analogous to the role played by the free representation of the superconformal algebra with central charge $3 / 2$ in the superstring theory. However, there are important differences between the representation theory of the spin- $4 / 3$ algebras and that of the spin-3/2 superVirasoro algebra of the superstring. In particular, the superconformal algebra has no parameters besides the central charge, whereas the split algebra has the structure constant $\lambda$.

It is easy to see that if we have two representations of the split algebra with currents and constants $\left\{G_{i}^{ \pm}, T_{i}, c_{i}, \lambda_{i}\right\}$ for $i=1$ and 2 , then we can form a new representation of the split algebra by tensoring them together only if $\lambda_{1}=\lambda_{2}$. The tensor-product algebra has currents and constants given by $\left\{G^{ \pm}=G_{1}^{ \pm}+G_{2}^{ \pm}, T=\right.$ $\left.T_{1}+T_{2}, c=c_{1}+c_{2}, \lambda=\lambda_{1}=\lambda_{2}\right\}$. If the $\lambda_{i}$ and $c_{i}$ are related by (C.5), then the new $\lambda$ and $c$ of the tensor-product algebra will satisfy an appropriately modified relation. The argument leading to (C.5) breaks down because the tensor-product algebra is not abelianly braided. In particular, new fractional powers appear among the regular terms of the OPEs. For example, the first regular term not shown in the $G^{+} G^{+}$OPE of eq. (C.1) is

$$
G^{+}(z) G^{+}(w) \sim(z-w)^{0}: G_{1}^{+} G_{2}^{+}:(w) .
$$

This term and its descendants all have integer powers of $(z-w)$. Though this is not a "cut," it nevertheless is a new fractional power, not included among the allowed exponents necessary for abelian braiding.

Using a simple anzatz for the four-point functions of the tensored currents $G^{+}(z)$ and $G^{-}(z)$, we can show explicitly how the appearance of this new "cut" relaxes the abelian associativity condition (C.5). First, we will assume this "cut" does not occur, and we will show how a simple algebraic argument recovers (C.5).

By $S L(2, \mathbf{R})$ invariance [14, we can write the four-point function (C.2) without 
any loss of generality as the sum of terms

$$
\mathcal{G}\left(z_{i}\right)=\sum_{j} k_{j} \mathcal{A}^{r_{j}} \mathcal{B}^{s_{j}} \mathcal{C}^{t_{j}}
$$

where $k_{j}$ are coefficients to be determined, and we have defined the combinations

$$
\begin{aligned}
\mathcal{A} & =\left(z_{1}-z_{2}\right)\left(z_{3}-z_{4}\right) \\
\mathcal{B} & =\left(z_{1}-z_{3}\right)\left(z_{2}-z_{4}\right) \\
\mathcal{C} & =\left(z_{1}-z_{4}\right)\left(z_{2}-z_{3}\right)
\end{aligned}
$$

In order for $\mathcal{G}$ to have the right behavior as its arguments approach infinity, the exponents must satisfy, for each $j$,

$$
r_{j}+s_{j}+t_{j}=-8 / 3
$$

Since only $\mathcal{A}$ vanishes as $z_{1} \rightarrow z_{2}$ or $z_{3} \rightarrow z_{4}$, its exponent is determined by the $G^{+} G^{+}$ or $G^{-} G^{-}$OPE in eq. (3.3), so that the $r_{j}$ must belong to the set $\{-4 / 3+n\}$ where $n$ is an integer. Similarly, the exponents of $\mathcal{B}$ and $\mathcal{C}$ are determined by the $G^{+} G^{-}$ OPE, implying $s_{j}, t_{j} \in\{-8 / 3+n\}$. We make the anzatz that the integers $n$ in the above sets satisfy $n \geq 0$. This assumption is not necessarily true in general; in the present case it can be verified using the Ward identity (C.4). With this assumption, there are 15 solutions for $\left\{r_{j}, s_{j}, t_{j}\right\}$ in these sets satisfying (C.10). Because $\mathcal{A}, \mathcal{B}$ and $\mathcal{C}$ are related by $\mathcal{A}-\mathcal{B}+\mathcal{C}=0$, there are only five independent terms in our expansion for $\mathcal{G}$ :

$$
\begin{array}{r}
\mathcal{G}=\frac{3 c_{0}}{8}\left[k\left(\frac{\mathcal{A}}{\mathcal{B}}\right)^{-4 / 3}+l\left(\frac{\mathcal{A}}{\mathcal{B}}\right)^{-1 / 3}+m\left(\frac{\mathcal{A}}{\mathcal{B}}\right)^{2 / 3}\right. \\
\left.+n\left(\frac{\mathcal{A}}{\mathcal{B}}\right)^{5 / 3}+p\left(\frac{\mathcal{A}}{\mathcal{B}}\right)^{8 / 3}\right] \mathcal{C}^{-8 / 3}
\end{array}
$$

Now if we take the limit $z_{3} \rightarrow z_{4}$ and compare the residues of the poles with those predicted by the OPEs (C.1), we find

$$
\begin{aligned}
\lambda^{2} & =k, \\
2 \lambda^{2} & =8 k+3 l .
\end{aligned}
$$

The limit $z_{2} \rightarrow z_{4}$ implies

$$
\begin{aligned}
3 c_{0} / 8 & =p \\
0 & =3 n+8 p \\
12 & =9 m+24 n+44 p
\end{aligned}
$$


and the remaining limit $z_{1} \rightarrow z_{4}$ implies

$$
\begin{aligned}
3 c_{0} / 8 & =k+l+m+n+p \\
0 & =-4 k-l+2 m+5 n+8 p \\
12 & =2 k-l+5 m+20 n+44 p .
\end{aligned}
$$

This overdetermined set of equations has a solution

$$
\begin{array}{ll}
k=\frac{1}{6}\left(8-c_{0}\right), & l=-\frac{1}{3}\left(8-c_{0}\right), \\
m=\frac{1}{6}\left(8+5 c_{0}\right), & n=-c_{0}, \quad p=3 c_{0} / 8
\end{array}
$$

only if $\lambda$ satisfies the abelian associativity condition (C.5).

The crucial assumption that enabled us to restrict the exponents $\left\{r_{j}, s_{j}, t_{j}\right\}$ to certain sets was that no fractional cuts besides those occurring in the singular terms on the right-hand side of the OPEs (C.1), appear among the "regular" terms. This, of course, is just the assumption of abelian braiding.

Let us now consider the tensor product algebra with currents $G^{ \pm}=G_{1}^{ \pm}+G_{2}^{ \pm}$, discussed above. New terms like (C.7) appear in the OPEs; these terms and their descendants all have integer powers of $(z-w)$. Though this is not a "cut," it nevertheless is a new fractional power, not included in our earlier set of allowed values for the exponents $\left\{r_{j}, s_{j}, t_{j}\right\}$. Allowing $r_{j}, s_{j}$ and $t_{j}$ to take on non-negative integer values (by our anzatz) implies that one new term appears in the expansion of $\mathcal{G}$ in addition to the five in eq. (C.11): $\left(3 c_{0} / 8\right) q \mathcal{C}^{-8 / 3}$. This results in a change in only the first residue equation in (C.12) to

$$
\lambda^{2}=k+q
$$

With this change, the residue equations have the same solution (C.15), but now with

$$
\lambda^{2}=\frac{8-c_{0}}{6}+q .
$$

Thus by varying $q$, we can achieve any value of $\lambda$ and still have an associative fourpoint function.

Similar observations hold for the full fractional superconformal algebra

$$
\begin{aligned}
G(z) G(w)= & \frac{1}{(z-w)^{8 / 3}}\left\{\frac{3 c_{0}}{4}+2(z-w)^{2} T(w)\right\} \\
& +\frac{\lambda}{(z-w)^{4 / 3}}\left\{G(w)+\frac{1}{2}(z-w) \partial G(w)\right\} .
\end{aligned}
$$

This algebra is non-abelianly braided since it has $(z-w)^{n-2 / 3}$ and $(z-w)^{n-1 / 3}$ cuts apparent among its singular terms. Thus, the relation derived above between the structure constant and central charge (C.5) will not necessarily be valid. Indeed, one 
of the alternative free boson plus cocycle representations of the fractional superconformal algebra (C.18) constructed in Appendix A violates (C.5), having $c_{0}=2$ but $\lambda=0$. Also, when we tensor together copies of the algebra (C.18) as in the discussion of the last paragraph, we also add in new "cuts" among the regular terms of the form $(z-w)^{0+n}$.

In summary, we have learned that the data necessary to specify a non-local chiral algebra consists not only of the singular pieces of the OPEs, but also of a list of all the fractional parts of the exponents of $(z-w)$ that may appear in the "regular" terms, as well. The abelian nature of the split algebra (C.1) allowed us to perform the analytic continuation necessary to derive a Ward identity, which in turn allowed us to relate the structure constant and the central charge. The non-split or tensored algebras have non-abelian braiding properties and do not, in general, satisfy any particular relation between $\lambda$ and $c$. In principle, though, if the exact form of the (non-abelian) braiding of the currents were known, one could solve the associativity conditions to find new relations between $\lambda$ and $c$. (For an example of this approach, see refs. [10, 11].)

\section{Appendix D}

In this appendix we will build the modular-invariant partition function for the closed $K=4$ FSS. This will primarily be a review of results obtained in refs. [2, 13]. We will use the partition function to predict the critical space-time dimension, to identify the bosonic and fermionic states of the FSS, and to determine the analog of the GSO projection.

We have argued in the body of this paper that the $K=4$ FSS consists of one free coordinate boson theory and one $\mathbf{Z}_{4}$ parafermion theory per space-time dimension. From this description we can readily deduce the form that the partition function for the $K=4$ fractional superstring must take. Each propagating bosonic world-sheet field $X^{\mu}$ contributes to the total one-loop partition function a factor

$$
\text { each boson } \Longrightarrow \frac{1}{\sqrt{\tau_{2}} \eta(q) \overline{\eta(q)}} \text {. }
$$

Here $\eta(q)$ is the Dedekind $\eta$-function defined in (2.21), $q=\mathrm{e}^{2 \pi i \tau}$ and $\tau \equiv \tau_{1}+i \tau_{2}$ is the torus modular parameter. Note that this factor includes the contributions from both holomorphic and anti-holomorphic (or left- and right-moving) components. The factor contributed by each world-sheet parafermion is

$$
\text { each parafermion } \Longrightarrow \mathcal{Z}_{m}^{j}(q)
$$

(the above is for left-moving parafermions; right-moving parafermions contribute the complex-conjugate). Here $\mathcal{Z}_{m}^{j}(q)$ are the parafermion characters whose expressions were derived earlier (2.22). 
It is convenient to introduce the string functions $23 c_{n}^{\ell}(q)$ by

$$
\mathcal{Z}_{m}^{j}(q) \equiv \eta(q) c_{2 m}^{2 j}(q) .
$$

From the parafermion field identities (2.3) and the expressions (2.22) for the parafermion characters, we see that the string functions obey

$$
c_{2 m}^{2 j}(q)=c_{-2 m}^{2 j}(q)=c_{2 m-4}^{4-2 j}(q) .
$$

Thus we can take as a basis of string functions the set $\left\{c_{0}^{0}, c_{0}^{2}, c_{0}^{4}, c_{1}^{1}, c_{1}^{3}, c_{2}^{2}, c_{2}^{4}\right\}$. Also, the string functions have power series expansions in $q$ starting with $c_{2 m}^{2 j}(q)=$ $q^{h(j, m)}(1+\cdots)$ where

$$
h(j, m)=\frac{2 j(j+1)-1}{12}-\frac{m^{2}}{4} \quad \text { for }|m| \leq j .
$$

Under the group of modular transformations generated by $T: \tau \rightarrow \tau+1$ and $S$ : $\tau \rightarrow-1 / \tau$, the string functions mix among themselves, forming a closed set. From eq. (D.5) it follows that the string functions are eigenfunctions under the $T$ modular transformation, while the $S$ transformation is given by [23, 24]

$$
c_{n}^{\ell}(-1 / \tau)=(-24 i \tau)^{-1 / 2} \sum_{L=0}^{4} \sum_{N=-3}^{4} \mathrm{e}^{i \pi n N / 4} \sin \left[\frac{\pi(\ell+1)(L+1)}{6}\right] c_{N}^{L}(\tau) .
$$

To construct the modular-invariant partition function for the closed FSS, we impose the conditions that there be a graviton in the closed string spectrum, and that no tachyonic states appear in the spectrum. Since the timelike and longitudinal polarizations of a massless graviton are not propagating degrees of freedom, we expect the partition function for the massless level to have contributions from only $D-2$ transverse dimensions. In order for the FSS partition function to have a chance of being modular invariant, it must be composed of products of the string functions which we have seen close among themselves under modular transformations. Thus, if only $D-2$ dimensions worth of states contribute at the massless level, this must also be true of the whole tower of massive states in the FSS. Therefore, for the closed $K=4$ fractional superstring, the propagating world-sheet field content consists of $D-2$ coordinate bosons and $D-2$ each of left- and right-moving parafermions. We therefore obtain the form for the total partition function:

$$
\mathcal{Z} \sim\left(\tau_{2}\right)^{1-D / 2} \sum \bar{c}^{D-2} c^{D-2}
$$

where $c$ stands for any $K=4$ string function. Note that the $\eta$-functions have cancelled between the bosonic and parafermionic contributions.

Consider for the moment the left-moving part of the $D$-dimensional FSS. The term in its partition function that includes the ground state is represented by the first term in the expansion of

$$
\left(c_{0}^{0}\right)^{D-2} \sim q^{-(D-2) / 12}(1+\ldots),
$$


as follows from the string function expansions (D.5). In the usual way, the power of $q$ in the partition function is interpreted as the mass squared of the state, while its coefficient is the state's multiplicity (i.e., the number of bosons minus the number of fermions). In this way we see that for $D>2$ the ground state of the FSS is tachyonic. Now let us consider the first excited state built from the ground state by the action of the parafermion energy operators $\epsilon^{( \pm 1)}$. This is the analog of the first excited state of the Neveu-Schwarz sector of the superstring, which describes a vector particle. Thus, the vector particle in the left-moving FSS partition function comes from the first term in the expansion of

$$
\left(c_{0}^{0}\right)^{D-3}\left(c_{0}^{2}\right) \sim q^{\frac{1}{3}-\frac{(D-2)}{12}}(1+\ldots) .
$$

Since the graviton in closed string theory comes from combining a left-moving and a right-moving vector particle, the masslessness of the graviton implies $D=6$. This is the basis of our claim that the $K=4$ FSS has critical dimension six.

Physically, we are only interested in string theories that are tachyon-free. This requires that tachyons must be projected out of the physical spectrum. Hence we are interested in constructing a partition function $\mathcal{Z}$ for closed string theories which contain the massless term (D.9) but no tachyonic term like (D.8). We find only one $D=6$ modular invariant partition function that satisfies these conditions [2]:

$$
\left(\tau_{2}\right)^{D / 2-1} \mathcal{Z}(q)=|A|^{2}+3|B|^{2}
$$

with

$$
\begin{aligned}
& A=4\left(c_{0}^{0}+c_{0}^{4}\right)^{3}\left(c_{0}^{2}\right)-4\left(c_{0}^{2}\right)^{4}-4\left(c_{2}^{2}\right)^{4}+32\left(c_{2}^{2}\right)\left(c_{2}^{4}\right)^{3} \\
& B=8\left(c_{0}^{0}+c_{0}^{4}\right)^{2}\left(c_{2}^{2}\right)\left(c_{2}^{4}\right)+16\left(c_{0}^{0}+c_{0}^{4}\right)\left(c_{0}^{2}\right)\left(c_{2}^{4}\right)^{2}-8\left(c_{0}^{2}\right)^{2}\left(c_{2}^{2}\right)^{2}
\end{aligned}
$$

Eq. (D.5) implies that the leading terms in a power series expansion in $q$ are $A \sim$ $q^{0}$ and $B \sim q^{1 / 2}$. Thus there are indeed no tachyons in this theory. The only contributions to the massless states are from the terms $4\left(c_{0}^{0}\right)^{3} c_{0}^{2}-4\left(c_{2}^{2}\right)^{4}$ in $A$. The first term we have already interpreted as the massless vector particle. The number of degrees of freedom of a massless vector particle in six dimensions is four, fixing the normalization of the partition function. The second term, appearing with a minus sign, must be interpreted as a space-time fermion. It is composed of $j=1$ spin fields in the parafermion theory, commonly denoted $\sigma_{ \pm 2}=\phi_{ \pm 1}^{1}$. The normalization of this term suggests that it is a massless space-time spin- $1 / 2$ Weyl fermion. In Sect. \# we will confirm this identification.

It turns out that there is an additional remarkable property shared by the expressions in (D.11). It can be shown [2, 13] that each of these new parafermionic string-function expressions vanishes as a function of $q$ :

$$
A(q)=B(q)=0
$$


This is interpreted as a sign of space-time supersymmetry cancellations in the fractional superstring spectrum of states.

From the expressions (D.10) and (D.11) for the partition function, we can immediately deduce a number of important properties of the full $K=4$ FSS Fock space.

First, and most obviously, we notice that the set of string functions $c_{2 m}^{2 j}$ which close on half-odd integer $j$ quantum numbers under the $S$ transformation (D.6) does not appear anywhere in the partition function. We deduce that the corresponding parafermion sectors with half-odd integer $j$ are projected out of the full string Fock space. Indeed, we have already assumed this fact in the discussion of Sect. 2.

Next, we notice that the term $4\left(c_{0}^{0}\right)^{3} c_{0}^{2}$ in the $A$ block of the partition function, identified as contributing to a space-time boson state in the left-moving theory, has the form of a product of parafermion sectors all with quantum numbers $m=0$. Thus it is natural to guess that in the light-cone gauge space-time bosons will have vertex operators proportional to

$$
\mathcal{B} \sim \prod_{i=1}^{D-2}\left(\phi_{0}^{j_{i}}\right),
$$

where $j_{i}=0,1$ or 2 . Any of the parafermion primary fields $\phi_{m}^{j}$ may, of course, be replaced by one of their descendant fields. Note that we are suppressing the worldsheet boson $\left(X^{\mu}\right)$ contributions to the vertex operators. Since the world-sheet bosons only give rise to states with space-time bosonic statistics, this suppression will not affect the identification of the statistics of vertex operators. By a similar argument following from the $A$ block term $-4\left(c_{2}^{2}\right)^{4}$, the fermions will have vertex operators proportional to

$$
\mathcal{F} \sim \prod_{i=1}^{D-2}\left(\phi_{ \pm 1}^{j_{i}}\right)
$$

where $j_{i}=1$ or 2 . According to the fusion rules (2.4) the parafermion $m$ quantum numbers add, so we see that (D.13) and (D.14) are consistent with the spin statistics connection. Indeed, these vertex operators satisfy the selection rules under fusion

$$
\begin{aligned}
& \mathcal{F} \cdot \mathcal{F} \rightarrow \mathcal{B} \\
& \mathcal{F} \cdot \mathcal{B} \rightarrow \mathcal{F} \\
& \mathcal{B} \cdot \mathcal{B} \rightarrow \mathcal{B},
\end{aligned}
$$

which follow from $m$ quantum number conservation and the parafermion field identifications (2.3). These selection rules serve to confirm our guess of the form of space-time boson and fermion states in the left-moving half of the FSS. In particular, we have identified the $m=0$ sector of the FSS Fock space as the analog of the superstring Neveu-Schwarz sector, and the $m= \pm 1$ sectors as the analog of the Ramond sector. In Sects. 1 and 5 we will confirm these identifications by finding the equations of motion satisfied by the massless physical states in each sector. 


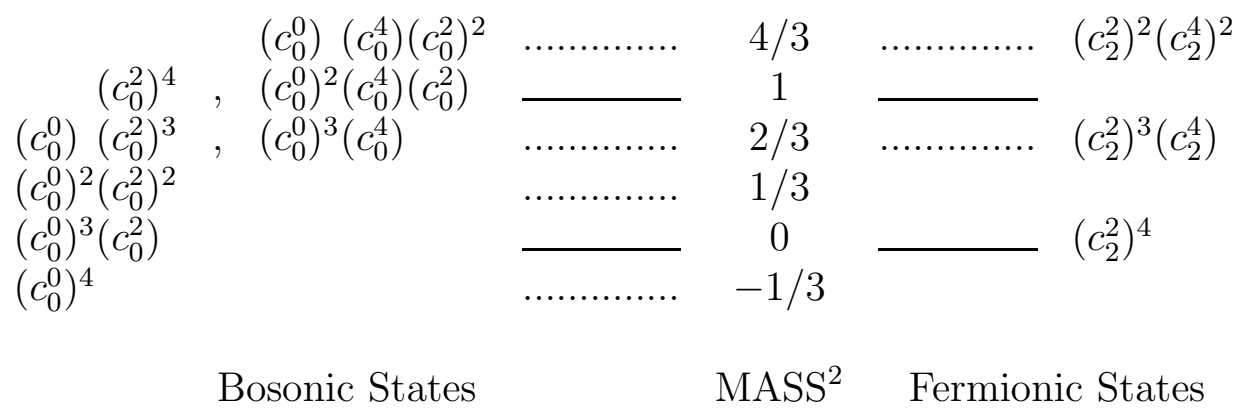

Figure 2: The low-lying mass levels for the $A$ block states of the $K=4$ FSS, along with the corresponding combinations of string functions which first get contributions from those levels. The masses of states in each level are indicated in units of the Planck mass. The mass levels removed by the GSO-like projection (i.e., whose string functions do not appear in the partition function) are indicated by dotted lines.

Note that not every state built by the action of $\epsilon^{( \pm 1)}$ on the bosonic or fermionic ground states (D.16) appears in the $A$ block partition function. For example, the bosonic ground state itself would contribute to the term $\left(c_{0}^{0}\right)^{4}$, a tachyonic state, which does not appear in (D.11). This projection, and similar ones at higher mass-levels in the FSS spectrum, are the analog of the GSO projection [16] in the superstring. The $A$ block partition function has the general expansion $A(q) \sim \sum_{N=0}^{\infty} a_{N} q^{N}$, whereas if arbitrary states built by the action of the $\epsilon^{( \pm 1)}$ fields on the bosonic and fermionic ground states appeared in the partition function, it would have the expansion $A(q) \sim$ $\sum_{n=-1}^{\infty} a_{n} q^{n / 3}$. Thus, in general, the analog of the GSO projection in the $K=4$ FSS removes $2 / 3$ of all the states from the bosonic and fermionic sector Fock spaces. The effect of this GSO-like projection on the low-lying states is shown in Fig. 2.

In the $A$ block of the FSS partition function, we have noted that the states fall into space-time bosonic and fermionic sectors. These sectors are built by the action of the energy operators $\epsilon^{( \pm 1)} \in\left[\phi_{0}^{1}\right]$ on the ground states

$$
\begin{aligned}
& \text { bosonic ground state } \sim \prod_{\mu=0}^{D-1} \mathbb{1}^{\mu} \text {, } \\
& \text { fermionic ground state } \sim \prod_{\mu=0}^{D-1} \sigma_{ \pm 2}^{\mu},
\end{aligned}
$$

where $\mathbb{1} \in\left[\phi_{0}^{0}\right]$ is the identity and $\sigma_{ \pm 2} \in\left[\phi_{1}^{1}\right]$ are the dimension $1 / 12$ spin fields. In the $A$ block, just as in the usual superstring, no other "mixed" sectors appear, built 
on ground states of the form

$$
\text { mixed ground state } \sim \prod_{\mu \in N} \prod_{\nu \in R} \mathbb{1}^{\mu} \sigma^{\nu}
$$

where $N$ and $R$ are disjoint sets of indices satisfying $N \cup R=\{0,1, \ldots, D\}$. This is another restriction placed on the full FSS Fock space by modular invariance. However, unlike the superstring, a certain "mixed sector" does appear in the $B$ block states of the FSS. The possible implications of the presence of $B$ block states are discussed in [12, 13]. This paper is restricted to an examination of the $A$ block states only.

\section{Appendix E}

We compute the critical central charge of a string theory with world-sheet fractional supersymmetry generated by the spin- $4 / 3$ currents $G^{+}$and $G^{-}$. These currents satisfy the split algebra with arbitrary central charge $c$ introduced in eqs. (3.3) and (C.1). Instead of rescaling $G^{+}$and $G^{-}$so that the structure constants are equal, as in eq. (3.3), we will leave them free in this appendix. This will allow us to discuss the hermiticity assignments of the currents more easily. Thus, the split algebra is

$$
\begin{aligned}
& G^{+}(z) G^{+}(w)=\frac{\lambda^{+}}{(z-w)^{4 / 3}}\left\{G^{-}(w)+\frac{1}{2}(z-w) \partial G^{-}(w)\right\}, \\
& G^{-}(z) G^{-}(w)=\frac{\lambda^{-}}{(z-w)^{4 / 3}}\left\{G^{+}(w)+\frac{1}{2}(z-w) \partial G^{+}(w)\right\}, \\
& G^{+}(z) G^{-}(w)=\frac{(1 / 2)}{(z-w)^{8 / 3}}\left\{\frac{3 c}{4}+2(z-w)^{2} T(w)\right\} .
\end{aligned}
$$

The associativity condition (C.5) for the split algebra then becomes

$$
\lambda^{+} \lambda^{-}=\frac{8-c}{6} .
$$

The modes of the $G^{ \pm}$currents and the enegry-momentum tensor $T$ satisfy the commutation relations

$$
\begin{aligned}
{\left[L_{m}, L_{n}\right] } & =(m-n) L_{m+n}+\frac{c}{12}\left(m^{3}-m\right) \delta_{m+n} \\
{\left[L_{m}, G_{r}^{ \pm}\right] } & =\left(\frac{m}{3}-r\right) G_{m+r}^{ \pm}
\end{aligned}
$$

and the GCRs

$$
\begin{gathered}
\sum_{\ell=0}^{\infty} c_{\ell}^{(-2 / 3)}\left[G_{ \pm \frac{q}{3}+n-\ell}^{ \pm} G_{\frac{2 \pm q}{3}+m+\ell}^{ \pm}-G_{ \pm \frac{q}{3}+m-\ell}^{ \pm} G_{\frac{2 \pm q}{3}+n+\ell}^{ \pm}\right]= \\
\frac{\lambda^{ \pm}}{2}(n-m) G_{\frac{2+2 q}{3}+n+m}^{\mp},
\end{gathered}
$$


and

$$
\begin{gathered}
\sum_{\ell=0}^{\infty} c_{\ell}^{(-1 / 3)}\left[G_{\frac{1+q}{3}+n-\ell}^{+} G_{-\frac{1+q}{3}+m+\ell}^{-}+G_{-\frac{2+q}{3}+m-\ell}^{-} G_{\frac{2+q}{3}+n+\ell}^{+}\right]= \\
L_{n+m}+\frac{3 c}{16}\left(n+1+\frac{q}{3}\right)\left(n+\frac{q}{3}\right) \delta_{n+m},
\end{gathered}
$$

when acting on a state with $\mathbf{Z}_{3}$ charge $q$.

Following the discussion of Sect. 3, the physical state conditions derived from this constraint algebra are

$$
\begin{aligned}
\left(L_{0}-v\right)\left|\phi_{\text {phys }}\right\rangle & =0, \\
L_{n}\left|\phi_{\text {phys }}\right\rangle & =0, \quad n>0, \\
G_{r}^{ \pm}\left|\phi_{\text {phys }}\right\rangle & =0, \quad r>0 .
\end{aligned}
$$

We can determine the critical values of the central charge and intercept by demanding the appearance of extra null states at those values of $c$ and $v$. As in the bosonic string and the superstring, extra null states can be taken as an indication of an enhanced gauge symmetry in the string theory.

Consider the spurious state with $\mathbf{Z}_{3}$ charge $q=1$

$$
|\phi\rangle=G_{-1 / 3}^{+}\left|\phi_{0}\right\rangle \text {. }
$$

It is built on a state with $\mathbf{Z}_{3}$ charge $q=0$, satisfying

$$
\begin{aligned}
L_{0}\left|\phi_{0}\right\rangle & =\left(v-\frac{1}{3}\right)\left|\phi_{0}\right\rangle, \\
L_{n+1}\left|\phi_{0}\right\rangle & =G_{n+2 / 3}^{ \pm}\left|\phi_{0}\right\rangle=0, \quad n \geq 0 .
\end{aligned}
$$

If $|\phi\rangle$ is a physical state, it will be null, since it is by construction spurious. The conditions (E.8) imply that $|\phi\rangle$ obeys all the physical state conditions (E.6) except one:

$$
\begin{aligned}
0 & =G_{1 / 3}^{-}|\phi\rangle=G_{1 / 3}^{-} G_{-1 / 3}^{+}\left|\phi_{0}\right\rangle \\
& =L_{0}\left|\phi_{0}\right\rangle=\left(v-\frac{1}{3}\right)\left|\phi_{0}\right\rangle
\end{aligned}
$$

where the second line follows by use of the GCR (E.5). Thus, we find a series of null states if $v=1 / 3$. Note that there is a second series with $\mathbf{Z}_{3}$ charge $q=-1$, found by exchanging $G^{+}$and $G^{-}$modes.

To fix $c$, we consider the series of spurious states with $q=1$, of the form

$$
|\phi\rangle=\left\{\alpha G_{-4 / 3}^{+}+\beta G_{-1 / 3}^{+} L_{-1}+\gamma G_{-1}^{-} G_{-1 / 3}^{-}\right\}\left|\phi_{0}\right\rangle,
$$


where $\left|\phi_{0}\right\rangle$ now satisfies

$$
\begin{aligned}
L_{0}\left|\phi_{0}\right\rangle & =-\left|\phi_{0}\right\rangle \\
L_{n+1}\left|\phi_{0}\right\rangle & =G_{n+2 / 3}^{ \pm}\left|\phi_{0}\right\rangle=0, \quad n \geq 0 .
\end{aligned}
$$

Eq. (E.10) is actually the most general spurious state with $q=1$ built from $\left|\phi_{0}\right\rangle$, since it is easy to show using the mode algebra (E.3)-(E.5) that other possible terms, like

$$
\left\{\delta L_{-1} G_{-1 / 3}^{+}+\epsilon G_{-1 / 3}^{+} G_{-2 / 3}^{-} G_{-1 / 3}^{+}\right\}\left|\phi_{0}\right\rangle
$$

can be expressed as linear combinations of the terms in (E.10). From the first condition in eq. (E.11), $|\phi\rangle$ has intercept $v=1 / 3$. It only remains to apply the rest of the physical state conditions to $|\phi\rangle$ to determine which values of $\alpha, \beta$, and $\gamma$ will give a new set of null states.

Using the commutation relations (E.3) $-($ E.5 $)$, one can compute the action of the positive modes of the currents on $|\phi\rangle$. Imposing the physical state conditions (E.6) yields the four independent conditions:

$$
\begin{aligned}
L_{1}|\phi\rangle=0 & \Longrightarrow 0=5 \alpha-4 \beta+2 \sqrt{2} \lambda^{-} \gamma \\
G_{1 / 3}^{-}|\phi\rangle=0 & \Longrightarrow 0=\alpha-2 \beta+4 \sqrt{2} \lambda^{-} \gamma \\
G_{4 / 3}^{-}|\phi\rangle=0 & \Longrightarrow 0=\left(\frac{9 c}{4}-4\right) \alpha-10 \beta-7 \sqrt{2} \lambda^{-} \gamma, \\
G_{1}^{+}|\phi\rangle=0 & \Longrightarrow 0=7 \sqrt{2} \lambda^{+} \alpha+4 \sqrt{2} \lambda^{+} \beta+\left(\frac{5 c}{2}-8-\lambda^{+} \lambda^{-}\right) \gamma .
\end{aligned}
$$

This system of equations has the solution

$$
\alpha=2 \sqrt{2} \lambda^{-} \gamma, \beta=3 \sqrt{2} \lambda^{-} \gamma, c=10, \lambda^{+} \lambda^{-}=-\frac{1}{3} .
$$

Thus, there are extra null states (since they are both physical and spurious) at $c=10$. Note that the associativity condition, eq. (E.2), is automatically satisfied by our solution.

At $c=10$ there are extra null states with $\mathbf{Z}_{3}$ charge $q=0$, as well. Consider the state

$$
|\phi\rangle=\alpha G_{-2 / 3}^{+}\left|\phi_{0}^{-}\right\rangle+\beta G_{-2 / 3}^{-}\left|\phi_{0}^{+}\right\rangle
$$

where $\left|\phi_{0}^{ \pm}\right\rangle$are primary states with $\mathbf{Z}_{3}$ charges $q= \pm 1$, which satisfy

$$
\begin{aligned}
L_{0}\left|\phi_{0}^{ \pm}\right\rangle & =-\frac{1}{3}\left|\phi_{0}^{ \pm}\right\rangle, \\
G_{0}^{+}\left|\phi_{0}^{+}\right\rangle & =b\left|\phi_{0}^{-}\right\rangle \\
G_{0}^{-}\left|\phi_{0}^{-}\right\rangle & =\frac{1}{b}\left(L_{0}-\frac{c}{24}\right)\left|\phi_{0}^{+}\right\rangle=-\left(\frac{c+8}{24 b}\right)\left|\phi_{0}^{+}\right\rangle, \\
L_{n+1}\left|\phi_{0}^{ \pm}\right\rangle & =G_{n+1 / 3}^{+}\left|\phi_{0}^{-}\right\rangle=G_{n+1 / 3}^{-}\left|\phi_{0}^{+}\right\rangle=0, \quad n \geq 0 .
\end{aligned}
$$


The $G_{0}^{+}$intercept $b$ is arbitrary. The $G_{0}^{-}$intercept follows from the previous two equations and the mode algebra $(\mathbb{E . 5})$. The physical state conditions then give

$$
\begin{aligned}
& G_{2 / 3}^{+}|\phi\rangle=0 \quad \Longrightarrow 0=-(c+8) \frac{\lambda^{+}}{\sqrt{2} b} \alpha+(c-4) \beta \\
& G_{2 / 3}^{-}|\phi\rangle=0 \quad \Longrightarrow 0=(c-4) \alpha+12 \frac{\lambda^{-} b}{\sqrt{2}} \beta
\end{aligned}
$$

which when solved subject to the condition (E.2) again give $c=10$.

So far this discussion has made no reference to the hermiticity properties of the currents. However, as discussed in Sect. 3.3 the hermiticity relations between mode operators play an important role in translating the quantum constraint equations into the physical state conditions $(\mathbb{E} .6)$. Also, it is natural to require the constraint operators to be hermitian, although there presumably is an interpretation of non-hermitian constraints as corresponding to propagation in time-dependent backgrounds.

Let us assume, then, that $G^{+}$and $G^{-}$are related by some hermiticity relations. It is not hard to show that, up to rescalings of the currents, the algebra (E.1) admits only four inequivalent hermiticity assignments:

$$
\begin{array}{rlll}
(i): & \left(G^{+}\right)^{\dagger}=G^{-} & \left(G^{-}\right)^{\dagger}=G^{+} & \lambda^{+}=\lambda^{-}, \\
(i i): & \left(G^{+}\right)^{\dagger}=-G^{-} & \left(G^{-}\right)^{\dagger}=-G^{+} & \lambda^{+}=-\lambda^{-} \\
(i i i): & \left(G^{+}\right)^{\dagger}=G^{+} & \left(G^{-}\right)^{\dagger}=G^{-} & \lambda^{+}=\lambda^{-} \\
(\text {iv }): & \left(G^{+}\right)^{\dagger}=G^{+} & \left(G^{-}\right)^{\dagger}=G^{-} & \lambda^{+}=-\lambda^{-}
\end{array}
$$

where in all cases $\lambda^{+}$can be taken to be a postive real number. When $c>8$, eq. (E.2) implies $\lambda^{+} \lambda^{-}<0$, so only the hermiticity assignments $(i i)$ or $(i v)$ are allowed. In these cases we can still construct non-split subalgebras, generated now by $G=G^{+}-G^{-}$. In both cases $(i i)$ and $(i v), G G \sim-1+\ldots$, showing that such FSCAs are necessarily non-unitary. The FSS involves copies of the FSCA at $c=2$, so cases $(i)$ and (iii) apply. Indeed, the free field representation at $c=2$ constructed in the body of the paper satisfies the hermiticity relations $(i)$.

We have determined the critical intercept and central charge of a spin- $4 / 3$ string to be $v=1 / 3$ and $c=10$. Note that this whole calculation could have been performed using the FSCA instead of the spin-4/3 algebra. The physical conditions would have been generated by the single current $G=G^{+}+G^{-}$. The same intercept and central charge emerge, using the sum of the $q=1$ and $q=-1$ null states described above. This result is in agreement with that obtained by consideration of the Kač determinant formula for the FSCA [8].

\section{References}

[1] See e.g. M.B. Green, J.H. Schwarz and E. Witten, Superstring Theory, Cambridge University Press (1987). 
[2] P.C. Argyres and S.-H.H. Tye, Phys. Rev. Lett. 67 (1991) 3339.

[3] A.B. Zamolodchikov and V.A. Fateev, Sov. Phys. J.E.T.P. 62 (1985) 215.

[4] V.G. Knizhnik and A.B. Zamolodchikov, Nucl. Phys. B247 (1984) 83.

[5] D. Kastor, E. Martinec and Z. Qiu, Phys. Lett. 200B (1988) 434.

[6] J. Bagger, D. Nemeschansky and S. Yankielowicz, Phys. Rev. Lett. 60 (1988) 389.

[7] F. Ravanini, Mod. Phys. Lett. A3 (1988) 397.

[8] P.C. Argyres, A. LeClair and S.-H.H. Tye, Phys. Lett. 253B (1991) 306.

[9] L. Brink and H.B. Nielsen, Phys. Lett. 45B (1973) 332.

[10] P.C. Argyres, J. Grochocinski and S.-H.H. Tye, Nucl. Phys. B367 (1991) 217.

[11] P.C. Argyres, J. Grochocinski and S.-H.H. Tye, Construction of the K=8 Fractional Supersymmetry Algebras, Cornell preprint CLNS 91/1126.

[12] K.R. Dienes and S.-H.H. Tye, Model-Building for Fractional Superstrings, Cornell preprint CLNS 91/1100.

[13] P.C. Argyres, K. Dienes and S.-H.H. Tye, Jacobi-like Identities for Parafermion Characters, Cornell/McGill preprint CLNS 91/1113, McGill/91-37.

[14] See e.g. A.A. Belavin, A.M. Polyakov and A.B. Zamolodchikov, Nucl. Phys. B241 (1984) 333.

[15] S.K. Yang, Nucl. Phys. B285 (1987) 183.

[16] F. Gliozzi, J. Scherk and D. Olive, Nucl. Phys. B122 (1977) 253.

[17] A.B. Zamolodchikov and V.A. Fateev, Theor. Math. Phys. 71 (1987) 451.

[18] See e.g. R.F. Streater and A.S. Wightman, PCT, Spin and Statistics, and All That, Benjamin/Cummings (1964).

[19] See e.g. P. Ginsparg, Applied Conformal Field Theory, in Les Houches, Session XLIX, 1988, Fields, Strings and Critical Phenomena, ed. by E. Breézin and J. Zinn-Justin, Elsevier Science Publishers (1989).

[20] L. Alvarez-Gaumé and E. Witten, Nucl. Phys.B234 (1983) 269; M.B. Green, J.H. Schwarz and P.C. West, Nucl. Phys. B254 (1985) 327; J.H. Schwarz, private communication. 
[21] R. Poghossian, Int. J. Mod. Phys. 6A (1991) 2005.

[22] S. Chung, E. Lyman and S.-H.H. Tye, Fractional Supersymmetry and Minimal Coset Models in Conformal Field Theory, Cornell preprint CLNS 91/1057, to appear in Int. J. Mod. Phys. A.

[23] V.G. Kac, Adv. Math. 35 (1980) 264; V.G. Kac and D. Peterson, Bull. AMS 3 (1980) 1057; Adv. Math.53 (1984) 125.

[24] D. Gepner and Z. Qiu, Nucl. Phys. B285 (1987) 423. 